INL/EXT-17-43388 Rev. 0

September 2017

\title{
Nuclear Energy Advanced Modeling and Simulation (NEAMS) Accident Tolerant Fuels High Impact Problem: Engineering Scale Models and Analysis
}
K. A. Gamble
J. D. Hales
D. M. Perez
G. Pastore

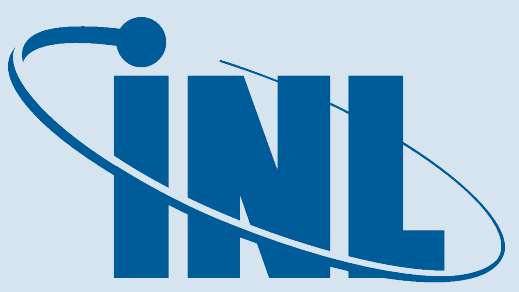

Idaho National Laboratory 


\section{NOTICE}

This information was prepared as an account of work sponsored by an agency of the U.S. Government. Neither the U.S. Government nor any agency thereof, nor any of their employees, makes any warranty, express or implied, or assumes any legal liability or responsibility for any third party's use, or the results of such use, of any information, apparatus, product, or process disclosed herein, or represents that its use by such third party would not infringe privately owned rights. The views expressed herein are not necessarily those of the U.S. Nuclear Regulatory Commission. 
Nuclear Energy Advanced Modeling and Simulation (NEAMS) Accident Tolerant Fuels High Impact Problem: Engineering Scale Models and Analysis
K. A. Gamble
J. D. Hales
D. M. Perez
G. Pastore

September 2017

Idaho National Laboratory

Fuel Modeling and Simulation Department

Idaho Falls, Idaho 83415

Prepared for the

U.S. Department of Energy

Office of Nuclear Energy

Under U.S. Department of Energy-Idaho Operations Office

Contract DE-AC07-05ID14517 


\begin{abstract}
As part of the Department of Energy's Nuclear Energy Advanced Modeling and Simulation program, an Accident Tolerant Fuel High Impact Problem was initiated at the beginning of fiscal year 2015 to investigate the behavior of $\mathrm{U}_{3} \mathrm{Si}_{2}$ fuel and iron-chromium-aluminum (FeCrAl) cladding under normal operating and accident reactor conditions. The High Impact Problem was created in response to the United States Department of Energy's renewed interest in accident tolerant materials after the events that occurred at the Fukushima Daiichi Nuclear Power Plant in 2011. The High Impact Problem is a multi national laboratory and university collaborative research effort between Idaho National Laboratory, Los Alamos National Laboratory, Argonne National Laboratory, and the University of Tennessee, Knoxville. This report summarizes the latest recommended material models to be used for engineering scale simulations based upon lower length scale analyses described elsewhere. Comparative analyses between traditional fuel rods $\left(\mathrm{U}_{3} \mathrm{Si}_{2} / \mathrm{Zircaloy}-4\right)$ and the proposed accident tolerant fuel rods $\left(\mathrm{UO}_{2} / \mathrm{FeCrAl}\right.$ and $\mathrm{U}_{3} \mathrm{Si}_{2}$ /Zircaloy-4) under normal operation, loss of coolant, and reactivity insertion conditions have been revisited using the latest state-of-the-art models implemented into the Bison fuel performance code. Sensitivity analyses have also been redone to take into account the latest knowledge on uncertain parameters.
\end{abstract}




\section{Contents}

\begin{tabular}{lll}
\hline & Introduction & 1
\end{tabular}

2 Overview of ATF Concepts 3

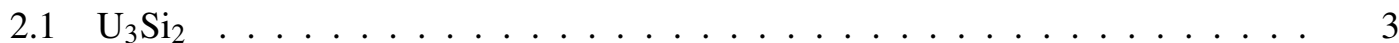

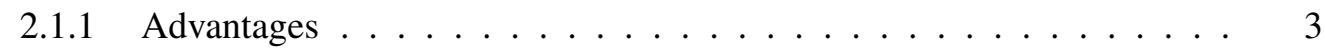

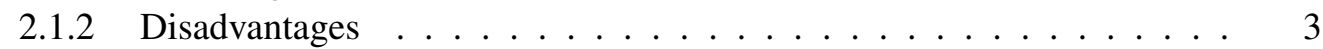

2.1 .3 Unknowns . . . . . . . . . . . . . . . . . . . 4

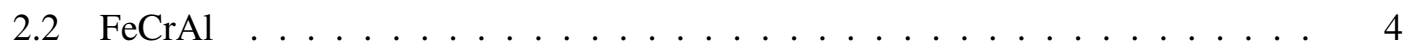

$2.2 .1 \quad$ Advantages . . . . . . . . . . . . . . . . . 4

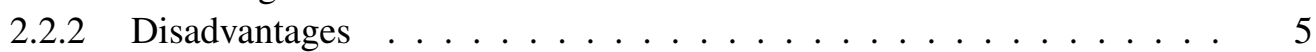

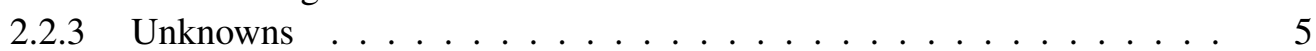

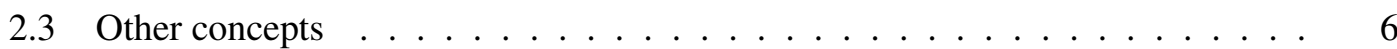

\begin{tabular}{lll}
\hline 3 & The Multiscale Approach & 7
\end{tabular}

4 Model Development 8

$4.1 \quad \mathrm{U}_{3} \mathrm{Si}_{2} \ldots \ldots \ldots \ldots \ldots \ldots \ldots$

4.1.1 Thermal and Mechanical Properties . . . . . . . . . . . . . 8

4.1.2 Swelling and Fission Gas Behavior . . . . . . . . . . . . . . . . . 11

4.1 .3 Thermal and Irradiation Creep . . . . . . . . . . . . . . . . . 17

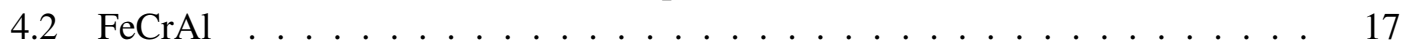

4.2.1 Thermal and Mechanical Properties . . . . . . . . . . . . . . . 18

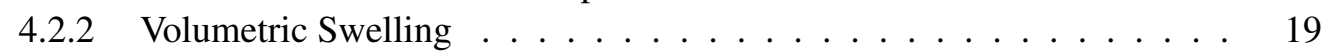

$4.2 .3 \quad$ Thermal and Irradiation Creep . . . . . . . . . . . . . . . . . 20

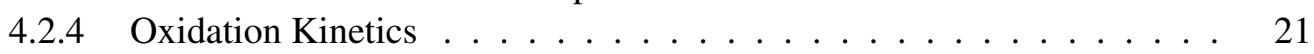

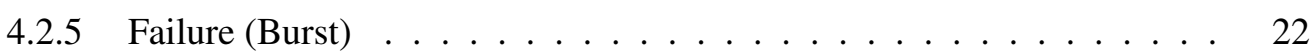

$\begin{array}{lll}5 & \text { Separate Effects Simulations } & 23\end{array}$

\begin{tabular}{lll}
\hline & Integral Rodlet Simulations & $\mathbf{2 4}$
\end{tabular}

6.1 Normal Operation . . . . . . . . . . . . . . . . . . . . . . . . 24

6.1 .1 Nominal Case . . . . . . . . . . . . . . . . . . . . . . 24

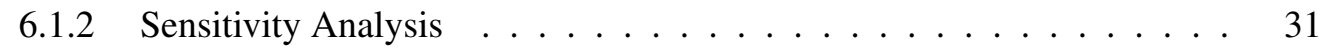

6.2 Loss of Coolant Accident . . . . . . . . . . . . . . . . . . . . . 37

$6.2 .1 \quad$ Nominal Case . . . . . . . . . . . . . . . . . . . . 37

6.2 .2 Sensitivity Analysis $\ldots \ldots \ldots \ldots$ 
6.3 Reactivity Insertion Accident $\ldots \ldots \ldots$. . . . . . . . . . . . . . . . . . . 48

$\begin{array}{lll}7 & \text { Collaborations } & 54\end{array}$

$7.1 \quad$ University of South Carolina . . . . . . . . . . . . . . . . . . . . . 54

7.2 Massachusetts Institute of Technology . . . . . . . . . . . . . . . . 54

7.3 Texas A\&M University $\ldots \ldots \ldots \ldots \ldots$. . . . . . . . . . . . . . . . . . . 54

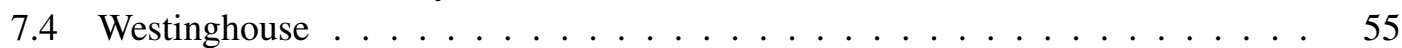

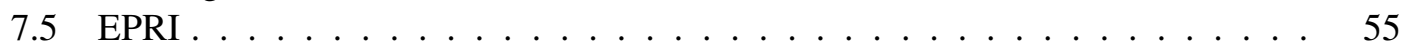

7.6 Advanced Fuels Campaign $\ldots \ldots \ldots \ldots$. . . . . . . . . . . . . 55

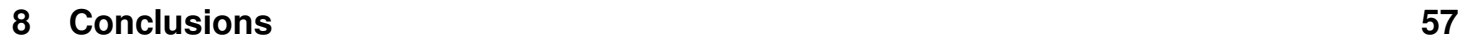

9 Future Work $\quad 59$

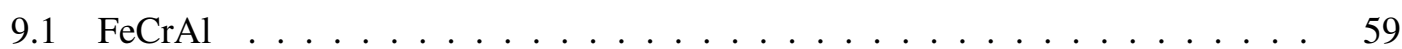

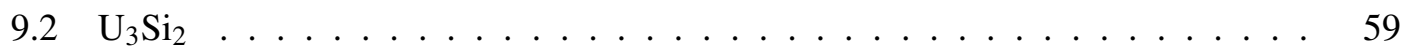

9.3 Silicon Carbide Cladding . . . . . . . . . . . . . . . . . . . . . 60

9.4 Coated Cladding $\ldots \ldots \ldots \ldots$

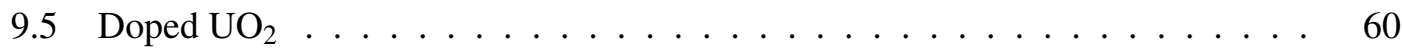

\begin{tabular}{lll}
\hline 10 Acknowledgements & 61
\end{tabular}

\begin{tabular}{ll}
\hline Bibliography & 62
\end{tabular} 


\section{Introduction}

In March 2011, a magnitude 9.0 earthquake struck off the coast of Japan. The earthquake and the associated tsunami resulted in tens of thousands of deaths, hundreds of thousands of damaged buildings, and a cost estimated to be in the hundreds of millions of dollars.

One consequence of the tsunami was the flooding of backup power generators at the Fukushima Daiichi Nuclear Power Station. The loss of power to coolant systems led to high temperatures, oxidation of Zr-based alloys, hydrogen production, melted fuel, and hydrogen explosions. As a result, a massive cleanup effort is underway at Fukushima Daiichi. The economic impacts, both those directly related to the cleanup and those affecting the nuclear energy sector generally, are significant.

Following the disaster, efforts to develop nuclear fuels with enhanced accident tolerance were begun by many nations, corporations, and research institutes. In the United States, the Department of Energy's Office of Nuclear Energy accelerated research on this topic as part of its Fuel Cycle Research and Development (FCRD) Advanced Fuels Campaign (AFC). One product of this work is Light Water Reactor Accident Tolerant Fuel Performance Metrics [1], a report by AFC that outlines a set of metrics that can be used to guide selection of promising accident tolerant fuel (ATF) concepts. Since that time, the AFC has begun irradiation of several ATF materials in Idaho National Laboratory's Advanced Test Reactor. The most promising ATF concepts are receiving increased focus and research funding. This work aims to assist industry in preparing one or more candidate fuels for insertion in a commercial reactor as a lead test rod or lead test assembly. The original goal was for insertion in 2022, but industry enthusiasm has moved that date earlier.

Given the aggressive development schedule, it is impossible to perform a comprehensive set of experiments to provide material characterization data. Therefore, the AFC plans to utilize computational analysis tools in an effort to understand the proposed materials.

The Nuclear Energy Advanced Modeling and Simulation (NEAMS) program in DOE has for some time been developing computational analysis tools. These include Bison [2-4] and Marmot [5], analysis tools tailored to nuclear fuel at the engineering scale and grain scale, respectively. Recently, NEAMS introduced what it calls High Impact Problems (HIPs) into its program plan. These HIPs are intended to make a significant advance in a particular area of nuclear power research in a short period of time (3 years or less). NEAMS chose an ATF project, which emphasizes utilizing Bison and Marmot to model proposed materials, as its first HIP. This report 
focuses on the multiscale and multiphysics development of engineering scale models of ATF fuel concepts, and the exercise of those models, accomplished through the ATF HIP. National laboratories participating in the HIP include Idaho National Laboratory (INL), Los Alamos National Laboratory (LANL), and Argonne National Laboratory (ANL). Contributions are also being made by university researchers, including at the University of Tennessee, Knoxville.

Accident tolerant fuels are expected to give similar or better performance during normal operation and improved performance in accident scenarios. Improved performance in accidents has been interpreted to mean allowing more time for cooling to be applied before unacceptable oxidation or melting occurs.

Of the many accident tolerant fuel concepts, two are the main focus of the NEAMS ATF HIP: uranium silicide $\left(\mathrm{U}_{3} \mathrm{Si}_{2}\right)$ fuel and iron-chromium-aluminum ( $\left.\mathrm{FeCrAl}\right)$ cladding. These were chosen through consultation with the Advanced Fuels Campaign as leading concepts, those seen as having a reasonable likelihood of being placed in a commercial reactor in the short term. $\mathrm{U}_{3} \mathrm{Si}_{2}$ is promising due to its higher thermal conductivity, which will give lower fuel temperatures during normal operation, and its higher uranium density, which has economic benefits. $\mathrm{FeCrAl}$ is promising due to a low oxidation rate and high strength.

The remainder of this report reviews these materials, models developed for analysis of these materials in reactor conditions, and insights related to the behavior of $\mathrm{U}_{3} \mathrm{Si}_{2}$ and $\mathrm{FeCrAl}$ concepts. A brief overview of the concepts comes first. Next is a review of the multiscale approach used to develop several of the material models. Then details of the engineering scale models are given. The report then reviews separate effects simulation and integral rodlet simulation results. The report ends with conclusions and an outline of future work. 


\section{Overview of ATF Concepts}

This chapter briefly introduces the two ATF candidates that are the focus of the NEAMS ATF HIP: $\mathrm{U}_{3} \mathrm{Si}_{2}$ fuel and $\mathrm{FeCrAl}$ cladding.

\section{$2.1 \mathrm{U}_{3} \mathrm{Si}_{2}$}

$\mathrm{U}_{3} \mathrm{Si}_{2}$ as a replacement for $\mathrm{UO}_{2}$ is being explored by the Advanced Fuels Campaign and Westinghouse.

\subsubsection{Advantages}

A main advantage of $\mathrm{U}_{3} \mathrm{Si}_{2}$ over $\mathrm{UO}_{2}$ fuel is the higher thermal conductivity. Thermal conductivity of $\mathrm{UO}_{2}$ is $\sim 2-5 \mathrm{~W} / \mathrm{m} / \mathrm{K}$ whereas the thermal conductivity of $\mathrm{U}_{3} \mathrm{Si}_{2}$ is $\sim 15-30 \mathrm{~W} / \mathrm{m} / \mathrm{K}$. This large difference results in lower fuel centerline temperatures and lower temperature gradients in the fuel pellet. Lower temperature gradients lower the likelihood of pellet cracking and relocation.

The high uranium density of $\mathrm{U}_{3} \mathrm{Si}_{2}\left(11.3 \mathrm{~g}-\mathrm{U} / \mathrm{cm}^{3}\right.$ versus $9.7 \mathrm{~g}-\mathrm{U} / \mathrm{cm}^{3}$ for $\mathrm{UO}_{2}$ [6] $)$ is economically attractive since it may enable higher burnup and longer cycle length. Uranium nitride fuel is attractive for the same reason, but its poor performance in water precludes its use without being combined with another material, such as $\mathrm{U}_{3} \mathrm{Si}_{2}$.

\subsubsection{Disadvantages}

Early work indicates that $\mathrm{U}_{3} \mathrm{Si}_{2}$ is more susceptible to chemical reaction than is $\mathrm{UO}_{2}$. For example, Harp et al. [7] report a "layered structure of corrosion products" on the surface of $\mathrm{U}_{3} \mathrm{Si}_{2}$ subjected to contact with water at $300^{\circ} \mathrm{C}$ for up to 24 hours. Furthermore, the authors report interdiffusion of $\mathrm{U}_{3} \mathrm{Si}_{2}$ and Zircaloy when tested at $800^{\circ} \mathrm{C}$ for 100 hours. Fe and $\mathrm{Cr}$ phases formed at the interface, with $\mathrm{ZrSi}_{2}$ and U-rich areas in Zircaloy also present. The authors indicate that further studies are needed, but it is clear that $\mathrm{U}_{3} \mathrm{Si}_{2}$ is more active than $\mathrm{UO}_{2}$. 
An exploration of increasing the oxidation resistance of $\mathrm{U}_{3} \mathrm{Si}_{2}$ was the subject of the study by Wood et al. [8]. Oxidation in $400^{\circ} \mathrm{C}$ air was shown to improve with the addition of aluminum. However, any alloying element decreases the uranium content per volume, diminishing the high uranium density advantage of the fuel. Oxidation of $\mathrm{U}_{3} \mathrm{Si}_{2}$ is further explored in [9].

Production of $\mathrm{U}_{3} \mathrm{Si}_{2}$ pellets has been completed in sufficient quantities to load experiments for irradiation in the Advanced Test Reactor. Scaling up the process to enable fueling whole assemblies and reactors remains to be done, with challenges still to be met [10].

\subsubsection{Unknowns}

Swelling of $\mathrm{U}_{3} \mathrm{Si}_{2}$ is a subject of some concern. Finlay et al. [11] reported significant swelling of $\mathrm{U}_{3} \mathrm{Si}_{2}$ under irradiation, and that data was used by Metzger et al. [12] to develop the first $\mathrm{U}_{3} \mathrm{Si}_{2}$ swelling model in Bison. The swelling in that model is quadratic in nature, giving significant swelling after a certain burnup. If the model is accurate, the swelling will induce high compressive stress in the fuel pellets. The effects of those stresses are unknown but could potentially involve pellet damage, particularly at the pellet edge where burnup is highest.

However, Finlay et al. attribute the high swelling to a change from a crystalline to an amorphous state. This change may not occur at reactor temperatures, and therefore the swelling may be considerably lower than originally assumed. More research, both experimental and computational, is underway to understand the phenomenon better.

Fuel swelling is connected to fission gas release, and just as there is uncertainty about swelling, there is uncertainty about the amount of fission gas that will be released from $\mathrm{U}_{3} \mathrm{Si}_{2}$ fuel. Experimental evidence is missing in this respect, and there is a need for experimental and theoretical work on fission gas behavior in $\mathrm{U}_{3} \mathrm{Si}_{2}$.

\subsection{FeCrAl}

Iron-chromium-aluminum alloy $(\mathrm{FeCrAl})$ has been proposed as a cladding material to replace Zircaloy. This concept is being pursued by the Advanced Fuels Campaign and General Electric.

\subsubsection{Advantages}

One of the most significant factors in the Fukushima Daiichi power plant accident was the oxidation of Zircaloy. Given that fact, perhaps the most attractive characteristic of FeCrAl cladding 
is its low oxidation rate. Oxidation rates for $\mathrm{FeCrAl}$ are approximately 1000 times lower than the oxidation rates of Zircaloy [13, 14]. The oxidation rates for $\mathrm{FeCrAl}$ are so low that a good approximation when modeling is that the $\mathrm{FeCrAl}$ cladding will not oxidize at all.

The stiffness of $\mathrm{FeCrAl}$ is roughly twice that of Zircaloy [14], while the yield stress is higher by a factor of four [13].

\subsubsection{Disadvantages}

The thermal neutron absorption cross section of $\mathrm{FeCrAl}$ is about ten times that of Zircaloy. This neutronic penalty necessitates thinner cladding, which is possible due to the higher strength of FeCrAl. However, thinner cladding, including the use of slightly larger pellets giving the same cold gap width, is not enough to compensate for the neutronic penalty. Enriching the fuel beyond the current 5\% limit appears to be necessary [14].

Current estimates are that $\mathrm{FeCrAl}$ cladding, with its neutronic penalty, will impose a fuel cost increase of $15-35 \%$ [13, 14].

A second disadvantage of $\mathrm{FeCrAl}$ cladding is the anticipated increase in tritium release to the coolant. The permeability of hydrogen in $\mathrm{FeCrAl}$ is about 100 times higher than its permeability in Zircaloy [15]. Mitigation strategies for this weakness are being considered.

\subsubsection{Unknowns}

Material data for $\mathrm{FeCrAl}$ under irradiation is just now becoming available. While reasonable expectations have been stated for the irradiation behavior, more testing is necessary to confirm the assumptions.

The thermal creep rate of $\mathrm{FeCrAl}$ is significantly lower than that of Ziracloy. A preliminary correlation for irradiation creep is available, and further experimental investigations are currently underway at Halden. It is also worth considering the possibility of using the creep rate as a design parameter. Perhaps slight alloying adjustments might be made that result in a creep rate that optimizes a particular behavior of the cladding.

Isotropic swelling of $\mathrm{FeCrAl}$ alloys under fast neutron flux is not yet characterized.

It is expected that $\mathrm{FeCrAl}$ will become harder and more brittle with irradiation [14]. The extent of the changes will have important consequences for the accident behavior of the cladding. Recent work indicates that burst $\mathrm{FeCrAl}$ tubes may open more fully than Zircaloy tubes [16]. It 
is possible that an embrittled cladding would open even more fully upon burst, increasing the likelihood of fuel being dispersed into the coolant.

\subsection{Other concepts}

Several other accident tolerant fuel concepts have been proposed. While not a primary focus of the ATF HIP, a few are mentioned here.

Silicon carbide cladding is being proposed due to low oxidation rates. However, challenges include dissolution in coolant [17], manufacturing and maintaining hermiticity [18], and joining [19].

Coated Zircaloy cladding is another possibility. This technology is being pursued by several institutions, including AREVA [20]. Challenges include manufacturing and the fact that initial work has focused on the exterior of the cladding, leaving the interior of the cladding unprotected.

Chromium-doped $\mathrm{UO}_{2}$ fuel has been under investigation for some time due to the presumption that larger grains will result in lower fission gas release. Recent work by researchers at Los Alamos National Laboratory and others has helped explain the effects of dopants. More research is needed to evaluate the competing effects of a larger grain size and a changed fission gas diffusivity. Chromium-doped $\mathrm{UO}_{2}$ is also reported to be a softer fuel, lessening harmful PCMI effects.

Fully ceramic microencapsulated (FCM) fuel has also been proposed (see [21] as an example). The FCM concept involves TRISO fuel particles embedded in a matrix, the whole taking on a geometry similar to that of $\mathrm{UO}_{2}$ pellets. FCM, like other accident tolerant fuel concepts, lacks detailed irradiated material behavior data and is likely to be more expensive than the current $\mathrm{UO}_{2} /$ Zircaloy fuel system. 


\section{The Multiscale Approach}

As previously mentioned, the aggressive deployment goals for ATF concepts make characterization of the material properties and behavior of ATF materials through extensive experimental investigation impossible. Early decisions are being based on engineering judgment, very limited experimental data, and computational modeling.

Computational modeling generally relies on experimental data to provide guidance for empirical or mechanistic descriptions of material behavior. Models for $\mathrm{UO}_{2}$ and Zircaloy in use today are based on decades of collected data, data both from dedicated experiments and from commercial use. In the case of $\mathrm{U}_{3} \mathrm{Si}_{2}$ and $\mathrm{FeCrAl}$, similar data does not exist, and a different approach is needed.

Taking thermal conductivity of fuel as an example, it is common to develop a correlation for thermal conductivity as a function of burnup. However, if data is not available, thermal conductivity degradation can be estimated based on grain-level behavior. Particularly, the effect of fission gas bubbles on thermal conductivity can be estimated given calculations performed at the scale of grains of fuel. This approach with Marmot has been demonstrated in the literature [2224] for $\mathrm{UO}_{2}$ fuel. This approach requires data from the atomistic scale as input to the grain-level calculations. Thus, it is truly a multiscale approach.

A similar approach has been taken for $\mathrm{U}_{3} \mathrm{Si}_{2}$ using Marmot and GRASS-SST [25]. Marmot [5] and VPSC [26] are likewise being used to develop predictive models for FeCrAl cladding. The following chapter shows some of the results of this multiscale modeling effort. 


\section{Model Development}

\section{1 $\mathrm{U}_{3} \mathrm{Si}_{2}$}

The material and behavior models obtained from the existing literature or developed based upon lower length scale modeling and incorporated into Bison to facilitate fuel performance analyses of $\mathrm{U}_{3} \mathrm{Si}_{2}$ fuel include thermal and mechanical properties, swelling and fission gas behavior, and thermal and irradiation creep. The details of the models are described in this chapter.

\subsubsection{Thermal and Mechanical Properties}

The thermal and mechanical properties of $\mathrm{U}_{3} \mathrm{Si}_{2}$ fuel include elastic properties, thermal conductivity, specific heat capacity, and thermal expansion.

\subsubsection{Elastic Properties}

The elastic properties of $\mathrm{U}_{3} \mathrm{Si}_{2}$ determines the mechanical response of the fuel under mechanical loading in the elastic regime. Limited experimental data exists regarding the temperature dependence of Young's modulus and Poisson's ratio, and the data that is available is based upon dispersion research reactor and not power reactor conditions. However, in the absence of other data, constant values of $140 \mathrm{GPa}$ and 0.17 for Young's modulus and Poisson's ratio, respectively, are used as per Metzger et al. [12].

\subsubsection{Thermal Conductivity}

The thermal conductivity of $\mathrm{U}_{3} \mathrm{Si}_{2}$ is a measure of its ability conduct heat and appears in Fourier's Law for heat conduction. Three different models have been added to Bison to account for the evolution of thermal conductivity as a function of temperature, temperature gradient, and fission density (burnup). 
The base empirical thermal conductivity model is a linear function of temperature based upon experiments by White et al. [27]:

$$
k=4.996+0.0118 T
$$

where $k$ is the thermal conductivity in $\mathrm{W} / \mathrm{m}-\mathrm{K}$ and $T$ is the temperature in $\mathrm{K}$. This model is valid from $300 \mathrm{~K}$ to $1773 \mathrm{~K}$.

The thermal conductivity degradation model for $\mathrm{U}_{3} \mathrm{Si}_{2}$ uses the unirradiated thermal conductivity calculated by Equation 4.1 to calculate the intrinsic thermal conductivity. The intrinsic thermal conductivity is then multiplied by two degradation factors due to contributions from intergranular and intragranular bubbles. The model is based upon rate theory calculations [28] for which a tricubic interpolation algorithm was developed for incorporation into Bison. The model is a function of temperature, local temperature gradient, and fission density (burnup). The ranges of applicability of the model are temperatures from $390 \mathrm{~K}$ to $1190 \mathrm{~K}$, temperature gradients from 0 to $160 \mathrm{~K} / \mathrm{mm}$ and fission densities from 0 to $2.5755 \times 10^{21}$ fissions $/ \mathrm{cm}^{3}$.

The intrinsic thermal conductivity is calculated as follows:

$$
k_{\text {in }}=\frac{k_{\text {white }}}{1-k_{\text {white }} \frac{R}{g}}
$$

where $k_{\text {white }}$ is thermal conductivity calculated by the White model, $R$ is the Kapitza resistance $\left(2.5 \mathrm{e}-8 \mathrm{~m}^{2}-\mathrm{K} / \mathrm{W}\right)$, and $g$ is the grain size (taken as $\left.35 \mu \mathrm{m}\right)$.

The modified Kapitza resistance is determined based upon the amount of grain boundary coverage:

$$
G B_{\text {cov }}=\frac{F C O V}{3000.0}
$$

where $G B_{c o v}$ is the grain boundary coverage, and $F C O V$ is computed by the tricubic interpolation calculation. The modified Kapitza resistance is then determined by:

$$
R^{\prime}=R\left(1-G B_{c o v}\right)^{0.86+0.3 \ln (R)}
$$

The intergranular factor is then computed by: 


$$
f_{\text {inter }}=\frac{1.0}{1.0-k_{\text {in }} \frac{R^{\prime}}{g_{g}}}
$$

where $g_{g}$ is the grain size specified in the GRASS-SST rate theory calculation. This value is taken as $5.0 \mu \mathrm{m}$. The intragranular factor is calculated by:

$$
f_{\text {intra }}=\frac{1.0-G S W b}{1.0+0.9 G S W b}
$$

where $G S W b$ is the intragranular gaseous swelling strain due to intragranular bubbles calculated by the tricubic interpolation calculation. Finally the thermal conductivity is then given as:

$$
k=k_{\text {in }} f_{\text {inter }} f_{\text {intra }}
$$

\subsubsection{Specific Heat Capacity}

The specific heat capacity of $\mathrm{U}_{3} \mathrm{Si}_{2}$ is measure of its ability to absorb heat per unit mass and is of particular importance during reactivity insertion accidents (RIA). The most recent model available in Bison is based upon the experiments of White et al. [6, 27]. In J/mol-K the specific heat is calculated via a linear function of temperature:

$$
c_{p}=140.5+0.02582 T
$$

The $c_{p}$ value is then converted into units of $\mathrm{J} / \mathrm{kg}-\mathrm{K}$ by dividing by the molar mass of $\mathrm{U}_{3} \mathrm{Si}_{2}$ given by $0.770258 \mathrm{~kg} / \mathrm{mol}$.

\subsubsection{Thermal Expansion}

According to Metzger et al. [12] the linear thermal expansion coefficient is reported in multiple locations within the literature. The value chosen by those authors and used in this work is a constant value valid from $298 \mathrm{~K}$ to $1473 \mathrm{~K}$ of $15.0 \times 10^{-6} \mathrm{~K}^{-1}$. 


\subsubsection{Swelling and Fission Gas Behavior}

The understanding of fission product swelling and fission gas release in $\mathrm{U}_{3} \mathrm{Si}_{2}$ is essential to predict the fuel performance of a fuel rod containing $\mathrm{U}_{3} \mathrm{Si}_{2}$ because it will directly influence the stress and strain states in both the fuel and cladding as well as the internal pressure of the rod. Three models have been developed for this purpose: empirical, rate theory, and coupled gas swelling and fission gas release. A densification model is also used that takes into account the sintering of the fuel early in life.

\subsubsection{Densification}

$\mathrm{U}_{3} \mathrm{Si}_{2}$ is expected to experience densification similar to $\mathrm{UO}_{2}$. Thus, the fuel densification is computed using the ESCORE empirical model [29] given by:

$$
\varepsilon_{D}=\Delta \rho_{0}\left(e^{\left(\frac{B u \ln (0.01)}{C_{D} B u_{D}}\right)}-1\right)
$$

where $\varepsilon_{D}$ is the densification strain, $\Delta \rho_{0}$ is the total densification that can occur (given as a fraction of theoretical density), $B u$ is the burnup, and $B u_{D}$ is the burnup at which densification is complete. For temperatures below $750^{\circ} \mathrm{C}$ the parameter $C_{D}$ is given by $7.2-0.0086(T-25)$; above $750^{\circ} \mathrm{C}$ the parameter is $1.0\left(T\right.$ in $\left.{ }^{\circ} \mathrm{C}\right)$.

\subsubsection{Empirical Model}

Since the data for $\mathrm{U}_{3} \mathrm{Si}_{2}$ is limited, an empirical expression for the swelling of $\mathrm{U}_{3} \mathrm{Si}_{2}$ was determined using data from Figure 3 of [30]. The swelling of fuel particles was calculated by Finlay using the results of miniplate irradiation tests. To convert Finlay's data (fission density) to FIMA, a value of $10.735 \mathrm{~g} / \mathrm{cm}^{3}$ was used as the heavy metal density, equivalent to $95 \%$ theoretical heavy metal density. Based on Finlay's data the volumetric strain can be written as a function of burnup:

$$
\frac{d V}{V}=3.8808 \times B u^{2}+0.79811 \times B u
$$

where $d V / V$ is the volumetric strain at a given burnup Bu. The burnup is in units of FIMA. The quadratic equation for the total volumetric strain is then decoupled into its solid and gaseous components. The solid swelling is a linear function of burnup based upon the data of Hofman [31] using the same conversion procedure from fission density to burnup given above: 


$$
\left(\frac{d V}{V}\right)_{\text {solid }}=0.34392 \times B u
$$

which results in a gaseous swelling contribution given by the following quadratic function of burnup:

$$
\left(\frac{d V}{V}\right)_{\text {gaseous }}=3.8808 \times B u^{2}+0.45419 \times B u
$$

\subsubsection{Rate Theory Gaseous Swelling Model}

The rate theory gaseous swelling model uses the same tricubic interpolation scheme as the thermal conductivity degradation model. In this case, the parameter called GSW representing the total gaseous swelling is calculated and used. The same ranges of applicability as the thermal conductivity degradation model apply to this model. The value calculated by this model is added to the solid swelling given by Equation 4.11 and the densification given by Equation 4.9 to determine the total volumetric change.

\subsubsection{Combined Gaseous Swelling and Fission Gas Release}

The combined gaseous swelling and fission gas release model handles fission gas swelling and release in $\mathrm{U}_{3} \mathrm{Si}_{2}$ under power reactor conditions. The model calculates the coupled fission gas swelling and release concurrently and is physically based. This model relies on the current understanding of microstructure and fission gas behavior in $\mathrm{U}_{3} \mathrm{Si}_{2}$, including the recent findings from lower-length scale modeling and the available experimental data. Based on the experimental evidence from [32], we assume $\mathrm{U}_{3} \mathrm{Si}_{2}$ remains crystalline at power reactor temperatures. We also assume both intra-granular and grain-boundary gas bubbles develop, as in $\mathrm{UO}_{2}$.

In order to mitigate the scarcity of experimental data, new physically based descriptions of specific processes can be informed with $\mathrm{U}_{3} \mathrm{Si}_{2}$ material parameters which have been extracted from lower-length scale modeling. Furthermore, the physical interpretation of some relevant processes differ from the interpretation in the existing $\mathrm{UO}_{2}$ model in Bison to better conform to the current understanding of fission gas behavior in $\mathrm{U}_{3} \mathrm{Si}_{2}$. These processes include:

1. Modeling of intra-granular bubble nucleation and re-solution based on the so-called homogeneous mechanisms 
2. Modeling intra-granular bubble growth considering absorption of vacancies by the bubbles and based on an adaptation of the Speight-Beere model.

Material parameters are taken from lower-length scale calculations for $\mathrm{U}_{3} \mathrm{Si}_{2}$, where available. Parameters for which specific $\mathrm{U}_{3} \mathrm{Si}_{2}$ values are not yet available are given acceptable values based on data for metals, theoretical considerations or the best fitting of model results to experimental data. The model presented here is an initial engineering-scale fission gas model for $\mathrm{U}_{3} \mathrm{Si}_{2}$ that incorporates state-of-the-art understanding and lower-length scale modeling data and will be progressively updated as new data become available.

The model accounts for nucleation of bubbles, re-solution of gas from bubbles to the matrix, and trapping of gas from the matrix into the bubbles. Fission gas transport from within the fuel grains to the grain faces is computed by the numerical solution of the diffusion equation in one-dimensional spherical geometry.

Nucleation and re-solution may occur by different mechanisms, i.e., heterogeneous and homogeneous [33]. Heterogeneous nucleation and re-solution refer to the creation of new bubble nuclei as a direct consequence of the interaction of fission fragments with the lattice and bubble destruction occurring en bloc by passing fission fragments, respectively. The homogeneous mechanisms account for the nucleation of bubbles by diffusion-driven interactions of dissolved gas atoms and re-solution occurring gradually by ejection of individual atoms. The dominant mechanisms depend upon the nature of the interactions between fission fragments and lattice (electronic or phononic). Based on [34], we assume the homogeneous mechanisms to dominate in $\mathrm{U}_{3} \mathrm{Si}_{2}$. The equations for the evolution of the intra-granular gas bubble number density and gas atom concentrations are:

$$
\begin{gathered}
\frac{d N}{d t}=v-\frac{b}{n-1} N \\
\frac{\partial c}{\partial t}=D \frac{1}{r^{2}} \frac{\partial}{\partial r}\left(r^{2} \frac{\partial c}{\partial r}\right)-g c+b m-2 v+\beta \\
\frac{\partial m}{\partial t}=+g c-b m+2 v
\end{gathered}
$$

where $N\left(\mathrm{~m}^{-3}\right)$ is the number density of intra-granular bubbles, $n$ is the number of gas atoms per bubble, $c$ and $m\left(\mathrm{~m}^{-3}\right)$ are the intra-granular gas concentration in the matrix and in the bubbles, respectively, $t(\mathrm{~s})$ the time, $D\left(\mathrm{~m}^{2} \mathrm{~s}^{-1}\right)$ the single-atom gas diffusion coefficient, $r(\mathrm{~m})$ the radial coordinate in the spherical grain, $\beta\left(\mathrm{m}^{-3} \mathrm{~s}^{-1}\right)$ the gas generation rate, $g\left(\mathrm{~s}^{-1}\right)$ the trapping rate, $b$ $\left(\mathrm{s}^{-1}\right)$ the re-solution rate. The coefficient 2 of the nucleation rate $v\left(\mathrm{~atm} \mathrm{~m}^{-3} \mathrm{~s}^{-1}\right)$, represents the fact that bubbles are nucleated as dimers. 
The nucleation rate is calculated as:

$$
\mathrm{v}=8 \pi D R_{s g} f_{n} c^{2}
$$

where $R_{s g}(\mathrm{~m})$ is the radius of a single fission gas atom and $f_{n}$ is the nucleation factor $(/)$, equal to $10^{-6}$ (e.g., [35]). This system of equations is solved with an advanced version of the PolyPole algorithm [36].

Intra-granular bubble growth is treated using a modified [37] model. The mechanical equilibrium of an intra-granular bubble, assumed to be spherical, is governed by the Young-Laplace equation

$$
p_{e q}=\frac{2 \gamma}{R_{b}}-\sigma_{h}
$$

where $p_{e q}(\mathrm{~Pa})$ is the equilibrium pressure, $\gamma\left(\mathrm{J} \mathrm{m}^{-2}\right)$ is the $\mathrm{U}_{3} \mathrm{Si}_{2}$ gas surface energy and $\sigma_{h}(\mathrm{~Pa})$ is the hydrostatic stress. In general, the bubbles are in a non-equilibrium state and tend to the equilibrium condition absorbing or emitting vacancies. The vacancy absorption/emission rate can be calculated starting from the approach in [37] as

$$
\frac{\mathrm{d} n_{i v}}{\mathrm{~d} t}=\frac{2 \pi D_{i g}^{v} \rho}{k T \zeta}\left(p-p_{e q}\right)
$$

with $n_{i v}(-)$ the number of vacancies per intra-granular bubble, $D_{i g}^{v}\left(\mathrm{~m}^{2} \mathrm{~s}^{-1}\right)$ the intra-granular vacancy diffusion coefficient, $\rho(\mathrm{m})$ is the radius of the equivalent Wigner-Seitz cell surrounding a bubble and influenced by the vacancy absorption/emission, $k\left(\mathrm{~J} \mathrm{~K}^{-1}\right)$ is the Boltzmann constant, $T(\mathrm{~K})$ is the local temperature, and $\zeta(-)$ is an dimensionless factor, which is calculated as [38]

$$
\zeta=\frac{10 \psi\left(1+\psi^{3}\right)}{-\psi^{6}+5 \psi^{2}-9 \psi+5}
$$

where $\psi=\frac{R_{b}}{\rho}$ is the ratio between the bubble and the cell radii. The approach presented here differs from the original proposed in [37] because it involves a 3D representation of the absorption/emission phenomena, rather than a 2D description, which better suits the absorption/emission of vacancies at grain boundaries. For the diffusion coefficients, $D$ and $D v_{i g}$, and the re-solution rate, $b$, we use values for $\mathrm{U}_{3} \mathrm{Si}_{2}$ from the atomistic work of [39] and [34], respectively. 
The pressure of the bubble is expressed as, considering a van der Waals gas,

$$
p=\frac{k T}{\Omega-\eta \omega} \eta
$$

where $\Omega\left(\mathrm{m}^{3}\right)$ is the vacancy volume, $\eta(-)$ is the ratio between $\bar{n}$ and $n_{i v}$, and $\omega\left(\mathrm{m}^{3}\right)$ is the van der Waals atomic volume for xenon.

The volume of intra-granular bubbles is calculated as

$$
V_{b}=n_{i v} \Omega
$$

such that the radius is evaluated considering spherical bubbles

$$
R_{b}=\sqrt[3]{\frac{3 V_{b}}{4 \pi}}
$$

The intra-granular swelling is calculated as the average volume of the bubble with respect to the bubble number density.

The formation of the high burnup structure is not currently represented in the model, due to the lack of data on this phenomenon in $\mathrm{U}_{3} \mathrm{Si}_{2}$.

The numerical solution of Eqs. 4.14 allows estimating the arrival rate of gas at the grain faces, providing the source term for the grain-face gas behavior module. This computes both the fission gas swelling and release through a direct description of the grain-face bubble development, including bubble growth and coalescence (which are reflected in fuel swelling), and the eventual inter-connection (leading to thermal fission gas release).

These conceptual steps and the related equations are identical to those applied in the existing $\mathrm{UO}_{2}$ model in Bison. However, the material parameters are specific to the $\mathrm{U}_{3} \mathrm{Si}_{2}$ model. Nevertheless, in this model an initial concentration of grain-face bubbles equal to $2 \cdot 10^{12}$ is employed. The value is one order of magnitude lower than the one employed in $\mathrm{UO}_{2}$, reflecting the lower bubble density noticeable in uranium silicide from the available experimental data [32].

The fractional volume grain-face fission gas swelling is given by

$$
\left(\frac{\Delta V}{V}\right)=\frac{1}{2} \frac{N_{g f}}{(1 / 3) r_{g r}}\left(\frac{4}{3} \pi \varphi(\Theta) R_{g f}^{3}\right)
$$


where $N_{g f}$ is the number density of grain-face bubbles per unit surface, $r_{g r}$ the grain radius, $\Theta$ the bubble semi-dihedral angle, $\varphi(\Theta)$ the geometric factor relating the volume of a lenticular-shape bubble to that of a sphere, which is $1-1.5 \cos (\Theta)+0.5 \cos ^{3}(\Theta)$, and $R_{g f}$ the bubble radius of curvature. The factor $1 / 2$ is introduced in Eq. 4.23 because a grain-face bubble is shared by two neighboring grains.

Bubble growth is calculated with the model of Speight and Beere [37], to describe the growth (or shrinkage) of grain-face bubbles as proceeding by absorption (or emission) of vacancies in grain boundaries, induced by the difference between the pressure of the gas in the bubble, $p(\mathrm{~Pa})$, and the mechanical equilibrium pressure, $p_{e q}(\mathrm{~Pa})$.

The approach is conceptually analogous to that applied for the growth and shrinkage of intragranular bubbles. The diffusion coefficient of vacancies at grain boundaries is estimated by multiplying the intra-granular one by a factor of $10^{7}$.

This approach computes the bubble growth rate from the rate of inflow of gas atoms and the rate of absorption (emission) of vacancies at the bubble. The combined effects of gas atom inflow and vacancy absorption (emission) are interactive, since the addition of fission gas atoms gives rise to a change in the bubble pressure, which affects the propensity of the bubble to absorb (or emit) vacancies. Given the volume, $V_{g f}$, of a lenticular bubble of circular projection, the bubble radius of curvature is calculated as

$$
R_{g f}=\left(\frac{3 V_{g f}}{4 \pi \varphi(\Theta)}\right)^{\frac{1}{3}}
$$

The process of grain-face bubble coalescence, which leads to a progressive decrease of the bubble number density throughout irradiation, is described with a model based on [40, 41]. According to this model, the rate of loss of bubbles by coalescence is given by

$$
\frac{d N_{g f}}{d t}=-\frac{6 N_{g f}^{2}}{3+4 N_{g f} A_{g f}} \frac{d A_{g f}}{d t}
$$

where $N_{g f}$ and $A_{g f}$ represent the number density and projected area of grain-face bubbles, respectively.

The release of fission gas to the fuel rod free volume after the inter-connection of grain-face bubbles and the consequent formation of pathways for gas venting to the fuel exterior (thermal release) is based on the principle of grain face saturation. More specifically, a saturation coverage concept is adopted that assumes once the fractional coverage, $F$, attains a saturation value, $F_{\text {sat }}$, the bubble number density and projected area obey the saturation coverage condition 


$$
F=N_{g f} A_{g f}=F_{s a t}
$$

where $N_{g f}$ is the bubble number density and $A_{g f}=\pi(\sin (\Theta))^{2} R_{g f}^{2}$ is the bubble projected area on the grain face. In absence of experimental data on the maximum grain-face bubble coverage in $\mathrm{U}_{3} \mathrm{Si}_{2}$, the theoretical value $F s a t=\pi / 4$ is used.

Eq. 4.26 implies that, after attainment of the saturation coverage, a fraction of the gas on the grain faces is released to the fuel exterior and thereby compensates for continuing bubble growth.

\subsubsection{Thermal and Irradiation Creep}

A preliminary model for thermal and irradiation creep of $\mathrm{U}_{3} \mathrm{Si}_{2}$ fuel based upon the work of Metzger [42] has been incorporated into Bison. Initial simulations have indicated that above a homologous temperature of $0.75 T_{\text {melt }}$ and a von Mises to shear modulus ratio greater than $10^{-5}$ unrealistically high creep rates are observed. Discussions with Metzger and other researchers at the University of South Carolina have indicated that additional work is currently being conducted to investigate and improve the model. Therefore, in the simulations presented in this work $\mathrm{U}_{3} \mathrm{Si}_{2}$ is treated as an elastic material. It is noted that for improved comparisons between $\mathrm{U}_{3} \mathrm{Si}_{2}$ and $\mathrm{UO}_{2}$ fuel performance a thermal and irradiation creep model for $\mathrm{U}_{3} \mathrm{Si}_{2}$ should be used as it may offset some of the gaseous swelling that occurs. However, due to the only available model in the literature currently undergoing revision, it was deemed appropriate to treat the $\mathrm{U}_{3} \mathrm{Si}_{2}$ fuel as elastic.

\subsection{FeCrAl}

The material and behavior models obtained from the existing literature or developed based upon lower length scale modeling and incorporated into Bison to facilitate fuel performance analyses of FeCrAl cladding include thermal and mechanical properties, volumetric swelling, oxidation kinetics, thermal and irradiation creep, and failure (burst). The details of the models are described in this chapter. In this work the laboratory-developed FeCrAl alloy known as $\mathrm{C} 35 \mathrm{M}$ [43, 44] is of particular interest. In the absence of data for certain properties of C35M, the published data for the commercially available alloy called APMT ${ }^{\mathrm{TM}}$ is used. 


\subsubsection{Thermal and Mechanical Properties}

The thermal and mechanical properties of $\mathrm{FeCrAl}$ cladding include elastic properties, yield stress (YS), ultimate tensile strength (UTS),thermal conductivity, specific heat, and thermal expansion.

\subsubsection{Elastic Properties}

The temperature dependent Young's modulus and Poisson's ratio of C35M were obtained from Thompson et al. [45] as a function of temperature:

$$
\begin{gathered}
E=-5.46 \times 10^{-5} T^{2}-3.85 \times 10^{-2} T+1.99 \times 10^{2} \\
v=3.85 \times 10^{-5} T+2.68 \times 10^{-1}
\end{gathered}
$$

where $E$ is the Young's modulus (GPa), $v$ is Poisson's ratio, and $T$ is the temperature $\left({ }^{\circ} \mathrm{C}\right)$. These equations are valid for temperatures ranging from $25-850^{\circ} \mathrm{C}$. In the absence of higher temperature data these equations are extrapolated at temperature higher than $850{ }^{\circ} \mathrm{C}$.

\subsubsection{Yield Stress and Ultimate Tensile Strength}

The yield stress and ultimate tensile strength (UTS) of C35M as a function of temperature are illustrated as piecewise functions in Figure 4.1 The plot is adapted from Figure 10 in Yamamoto et al. [44]. At high temperatures it is observed that little ductility remains and failure will occur in a relatively brittle fashion. Irradiation effects on the yield strength and UTS are not taken into account.

Yamamoto's data only covers temperatures ranging from 300 to $1000 \mathrm{~K}$ [44]. Based on research by Yano et al. [46] on other ferritic and martensitic steels, there are distinct temperature dependent regions (low, mid, high) of the UTS. In the low temperature region the UTS drops relatively slowly with increasing temperature. In the midrange temperatures there is a rapid decrease in the UTS as temperature increases. The high temperature region results in a slow reduction of the UTS to approximately zero at the melting point. Using these observations on other alloys, an additional data point of a UTS of zero was added to Yamamoto's data at the melting point of C35M $(1773 \mathrm{~K})$. Since the yield stress approaches the UTS at the midrange temperatures, the yield stress is also set to zero at the melting point. 


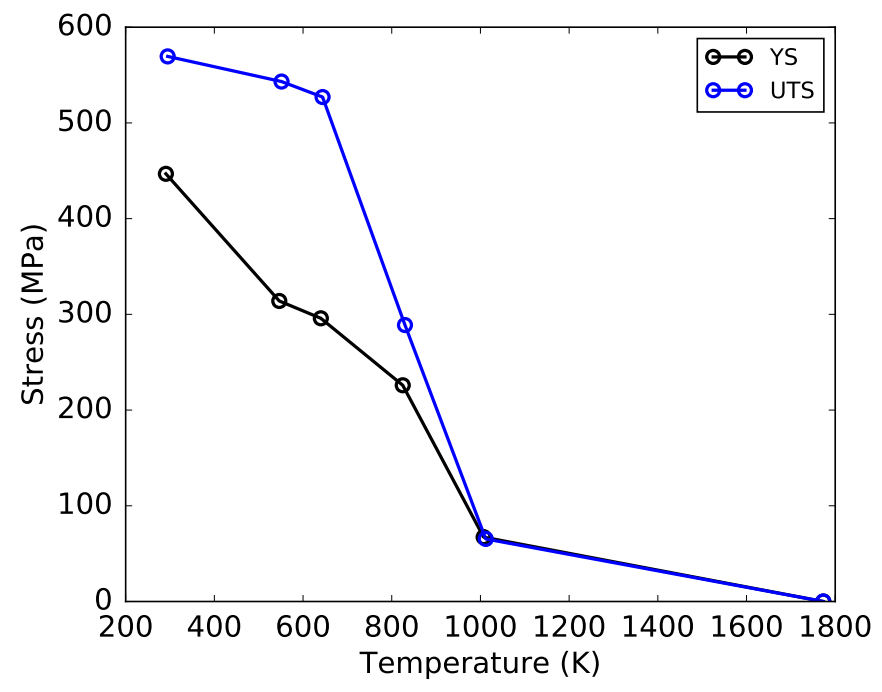

Figure 4.1: Yield stress (YS) and Ultimate Tensile Strength (UTS) as a function of temperature for $\mathrm{FeCrAl}$ alloy $\mathrm{C} 35 \mathrm{M}$. The UTS is set to zero at the melting point of $\mathrm{C} 35 \mathrm{M}$.

\subsubsection{Thermal Conductivity and Specific Heat}

The thermal conductivity and specific heat of $\mathrm{APMT}^{\mathrm{TM}}$ used in this analysis (in the absence of thermal property data of $\mathrm{C} 35 \mathrm{M}$ ) are obtained from the datasheet published by the manufacturer Sandvik AB [47]. The tabulated values are plotted in Figure 4.2 as a piecewise linear function. The non-monotonic trend of specific heat with temperature is adopted here for conformity with the data but will require further investigation in the future.

\subsubsection{Thermal Expansion}

The coefficient of linear thermal expansion adopted for C35M was taken from the APMT ${ }^{\mathrm{TM}}$ datasheet. The mean thermal expansion values are shown in Table 4.1. Since Bison requires the instantaneous thermal expansion coefficient, the methodology of Niffenegger and Reichlin [48] is employed to ensure correct results.

\subsubsection{Volumetric Swelling}

As a preliminary approach for the swelling of $\mathrm{C} 35 \mathrm{M}$, an upper bound swelling rate provided by Terrani et al. [49] of $0.05 \%$ per dpa is considered. Using the same conversion factor as for irradiation creep $\left(1 \times 10^{25}\right.$ neutrons $\left./ \mathrm{m}^{2}=0.9 \mathrm{dpa}\right)$ and integrating over time the volumetric swelling strain is given by: 


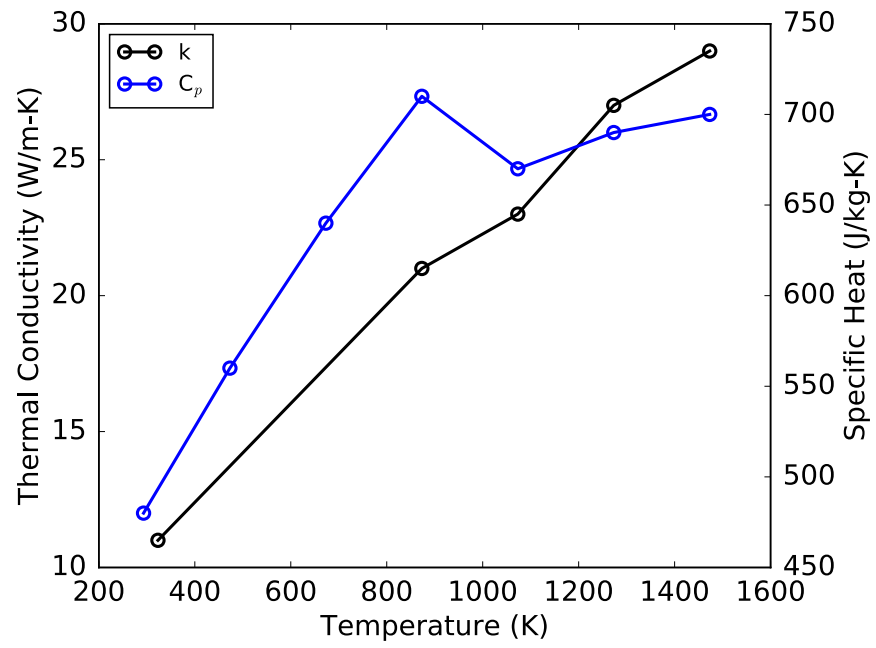

Figure 4.2: Thermal conductivity $(\mathrm{k})$ and specific heat $\left(\mathrm{C}_{p}\right)$ piecewise linear functions of temperature for Kanthal APMT ${ }^{\mathrm{TM}}$.

Table 4.1: Temperature dependent coefficient of thermal expansion (CTE) of Kanthal APMT ${ }^{\mathrm{TM}}$.

\begin{tabular}{ll}
\hline Temperature Range $\left({ }^{\circ} \mathrm{C}\right)$ & CTE $(\mu \mathrm{m} / \mathrm{m}-\mathrm{K})$ \\
\hline $20-250$ & 12.4 \\
$20-500$ & 13.1 \\
$20-750$ & 13.6 \\
$20-1000$ & 14.7 \\
$20-1200$ & 15.4 \\
\hline
\end{tabular}

$$
\varepsilon=4.5 \times 10^{-29} \Phi
$$

where and $\Phi$ is the fast neutron fluence (neutrons $/ \mathrm{m}^{2}$ ).

It should be noted that the incubation behavior of swelling is not considered at this time and should be included in the future as relevant data becomes available.

\subsubsection{Thermal and Irradiation Creep}

The recommended models for thermal and irradiation creep are given by Terrani et al. [49]. These are based on very recent experimental data from in-pile and out-of-pile creep tests performed at Halden and at ORNL. Thermal creep takes the form of the Norton creep law 


$$
\dot{\varepsilon}=2.89 \times 10^{-36} \sigma^{5.5} \exp \left(\frac{-29709}{T}\right)
$$

at temperatures below $873 \mathrm{~K}$. Above this temperature the correlation proposed by Saunders et al. [50] is used:

$$
\dot{\varepsilon}=5.96 \times 10^{-27} \sigma^{5.5} \exp \left(\frac{-47136}{T}\right)
$$

where $\dot{\varepsilon}$ is the creep rate $\left(\mathrm{s}^{-1}\right), \sigma$ is the effective stress $(\mathrm{Pa})$ and $T$ is the temperature $(\mathrm{K})$. The recommended irradiation creep coefficient [49] is $5 \times 10^{-6}$ per MPa per dpa. By utilizing a conversion factor of $1 \times 10^{25}$ neutrons $/ \mathrm{m}^{2}=0.9 \mathrm{dpa}$ [51], a correlation for irradiation creep can be derived:

$$
\dot{\varepsilon}=4.5 \times 10^{-31} \sigma \phi
$$

where $\sigma$ is the effective stress (MPa) and $\phi$ is the fast neutron flux (neutrons $/ \mathrm{m}^{2}-\mathrm{s}$ ).

\subsubsection{Oxidation Kinetics}

One of the advantages of $\mathrm{FeCrAl}$ alloys over zirconium-based alloys is their increased oxidation resistance. Recent autoclave experiments under PWR, BWR-HWC (hydrogen water chemistry), and BWR-NWC (normal water chemistry) conditions were completed by Terrani et al. [52]. Here, only the PWR and BWR-NWC cases are of interest. The experiments were conducted at normal operating temperatures $330^{\circ} \mathrm{C}$ and $290^{\circ} \mathrm{C}$ for PWR and BWR, respectively. Parabolic oxide growth kinetics govern the mass gain as a result of the formation and growth of the chromium rich chromite $\left(\mathrm{FeCr}_{2} \mathrm{O}_{4}\right)$ layer:

$$
w=k \sqrt{t}
$$

where $k$ is the parabolic oxidation rate constant $\left(\mathrm{mg} / \mathrm{cm}^{2}-\mathrm{h}^{1 / 2}\right)$ and $\mathrm{t}$ is the time (hr). The thickness of the chromite layer is then given by:

$$
\delta=\frac{w}{\rho_{o x}}
$$

where $\rho_{o x}$ is assumed to be the density of oxygen in chromite $\left(1440 \mathrm{~kg} \mathrm{~m} /{ }^{3}\right)[52]$.

The parabolic rate constants used from [52] in the Bison model are $3.96 \times 10^{-3}$ and $4.51 \times 10^{-4}$ $\mathrm{mg} / \mathrm{cm}^{2}-\mathrm{h}^{1 / 2}$ for PWR and BWR-NWC coolant conditions, respectively. These correspond to the $\mathrm{FeCrAl}$ alloy with composition of $\mathrm{Fe}-13 \mathrm{Cr}-4 \mathrm{Al}$ which most closely represents the $\mathrm{C} 35 \mathrm{M}$ 
alloy of interest in this work. Note that these rate constants are independent of temperature. Therefore, the mass gain and oxide thickness in this model are only dependent upon the irradiation time. As new data is becomes available the temperature dependence of the oxidation rate constant will be taken into account. In the description of the oxidation model only the formation of the oxide thickness was presented. It should be noted that Terrani et al. [52] state in addition to the formation of an oxide scale, additional metal in $\mathrm{FeCrAl}$ alloys will dissolve into the water, which results in a further reduction of the overall cladding thickness. This dissolution process does not occur in zirconium-based alloys. In this work, the formation of the oxide scale is the important mechanism as the amount of volatile hydrogen gas produced can be correlated to the thickness of the oxide.

\subsubsection{Failure (Burst)}

In early FY17 a failure criterion for FeCrAl claddings was developed based upon the experiments completed by Massey et al. [16]. The failure criterion is represented by a burst stress calculated by:

$$
\sigma_{\text {burst }}= \begin{cases}\text { Ultimate Tensile Strength, } & \text { for } T \leq 796.8 \mathrm{~K} \\ 28440.98 \mathrm{e}^{-0.005588 T}, & \text { for } T>796.8 \mathrm{~K}\end{cases}
$$

Further details of the development of this model can be found in Gamble et al. [53]. 


\section{Separate Effects Simulations}

Separate effects simulations examine the effects of individual material models on fuel performance parameters (e.g., temperatures, stresses, strains) of interest with minimal influence of other models. In some cases separate effects tests look at the behavior of one model given the inclusion of another model (e.g., examining the effect of oxidation on burst behavior). In other cases separate effects tests can be used as a method of verification to ensure sure that the solution obtained matches what would be calculated analytically. Separate effects simulations have been completed in earlier reports for $\mathrm{FeCrAl}$ [54] and $\mathrm{U}_{3} \mathrm{Si}_{2}$ [55]. In those reports comparisons between $\mathrm{FeCrAl}$ and Zircaloy-4 and $\mathrm{U}_{3} \mathrm{Si}_{2}$ and $\mathrm{UO}_{2}$ were completed respectively. Tables 5.1 and 5.2 provide a summary of conclusions. The reader is encouraged to read the previously published reports for additional information.

Table 5.1: Conclusions drawn from separate effects tests between $\mathrm{FeCrAl}$ and Zircaloy-4

\begin{tabular}{ll}
\hline Separate Effects Test & Conclusions \\
\hline Elasticity & FeCrAl experiences smaller strains but larger stresses \\
compared to Zircaloy-4. & FeCrAl experiences significantly smaller thermal creep \\
Thermal Creep & FeCrains compared to Zircaloy-4. \\
Thermal Conductivity & behavior as a function of temperature. \\
FeCrAl experiences significantly lower oxide thickness \\
Oxidation
\end{tabular}

Table 5.2: Conclusions drawn from separate effects tests between $\mathrm{U}_{3} \mathrm{Si}_{2}$ and $\mathrm{UO}_{2}$.

\begin{tabular}{ll}
\hline Separate Effects Test & Conclusions \\
\hline Thermal Conductivity & $\mathrm{U}_{3} \mathrm{Si}_{2}$ has higher thermal conductivity values than \\
& $\mathrm{UO}_{2}$ while taking into account degradation. \\
& $\mathrm{U}_{3} \mathrm{Si}_{2}$ experiences significantly larger strains due to \\
Volumetric Swelling & $\mathrm{gaseous}$ fission products when compared to $\mathrm{UO}_{2}$. \\
& $\mathrm{U}_{3} \mathrm{Si}_{2}$ has similar FGR at low temperatures and signifi- \\
& cantly larger FGR at high temperatures when compared \\
& $\mathrm{UO}_{2}$. \\
\hline
\end{tabular}




\section{Integral Rodlet Simulations}

In contrast to separate effects simulations, integral simulations investigate the evolution of fuel performance parameters when utilizing all of the existing material and behavior models for $\mathrm{U}_{3} \mathrm{Si}_{2}$. In previous publications integral rodlet simulations including sensitivity analyses have been completed for $\mathrm{FeCrAl}$ and $\mathrm{U}_{3} \mathrm{Si}_{2}[56-59]$. Since then, the more mechanistic lower length scale informed models have been incorporated into Bison. Therefore, the integral rod(let) tests are revisited and new sensitivity analyses are completed here. In this chapter the descriptions and results of four different types of scenarios are provided including, normal operation, loss of coolant accident, and reactivity insertion accident.

\subsection{Normal Operation}

\subsubsection{Nominal Case}

Recall that the definition of what qualifies a material as accident tolerant given by Bragg-Sitton et al. [1] states that a material must provide increased response time during postulated accident conditions with similar or improved performance during normal operation. Therefore, the first integral rodlet analysis was completed during normal operating PWR conditions. In this analysis three different 10 pellet rodlets $\left(\mathrm{UO}_{2} / \mathrm{Zircaloy}-4, \mathrm{UO}_{2} / \mathrm{FeCrAl}, \mathrm{U}_{3} \mathrm{Si}_{2} / \mathrm{Zircaloy}-4\right)$ were subjected to a power history consisting of a linear ramp from zero to $25 \mathrm{~kW} / \mathrm{m}$ over 10000 seconds and held constant for $\sim 3.2$ years. The fuel column was smeared (i.e., no dishes or chamfers on the pellets) with an initial density $95 \%$ of theoretical. The rodlet diameter (i.e., cladding outer diameter) and as manufactured fuel-to-clad gap was the same in all rodlets. To take into account the necessity for thinner cladding when using FeCrAl to overcome the neutronic penalty imposed by the increased thermal neutron absorption cross-section, the cladding thickness was reduced in the $\mathrm{UO}_{2} / \mathrm{FeCrAl}$ rodlet while the fuel outer diameter was increased. Table 6.1 summarizes the details of the three rodlets.

The results of the normal operation analysis for the three different cases are shown in Figures 6.1 through 6.6. Figure 6.1 shows the time evolution of the fuel centerline temperature for the three different rodlets analyzed. As expected, the $\mathrm{U}_{3} \mathrm{Si}_{2}$ rod achieves lower temperature during the entire irradiation time because of the significantly larger thermal conductivity of the fuel. The gradual decrease in temperature in the two Zircaloy- 4 clad rods is caused by the creeping down 
Table 6.1: Rodlet specifications for normal operation simulations

\begin{tabular}{lccc} 
& $\mathrm{UO}_{2} / \mathrm{Zircaloy}-4$ & $\mathrm{UO}_{2} / \mathrm{FeCrAl}$ & $\mathrm{U}_{3} \mathrm{Si}_{2} / \mathrm{Zircaloy}-4$ \\
Rodlet & Rodlet & Rodlet \\
\hline Number of pellets & 10 & 10 & 10 \\
Fuel enrichment $(\%)$ & 5 & 5 & 5 \\
Fuel density $\left(\mathrm{kg} / \mathrm{m}^{3}\right)$ & 10431.0 & 11590.0 & 10431.0 \\
Pellet length $(\mathrm{mm})$ & 11.86 & 11.86 & 11.86 \\
Pellet outer diameter $(\mathrm{mm})$ & 8.19 & 8.57 & 8.19 \\
Radial gap width $(\mu \mathrm{m})$ & 80 & 80 & 80 \\
Clad thickness $(\mathrm{mm})$ & 0.575 & 0.385 & 0.575 \\
Rodlet length $(\mathrm{mm})$ & 150 & 150 & 150 \\
Rodlet diameter $(\mathrm{mm})$ & 9.5 & 9.5 & 9.5 \\
Initial fill pressure $(\mathrm{MPa})$ & 2 & 2 & 2 \\
Initial fill gas & $\mathrm{Helium}$ & $\mathrm{Helium}$ & $\mathrm{Helium}$ \\
Plenum height $(\mathrm{mm})$ & 26 & 26 & 26 \\
Initial fuel grain radius $(\mu \mathrm{m})$ & 10 & 10 & 10 \\
Coolant inlet mass flux $\left(\mathrm{kg} / \mathrm{m}^{2}-\mathrm{s}\right)$ & 3800 & 3800 & 3800 \\
Coolant inlet temperature $(\mathrm{K})$ & 580 & 580 & 580 \\
Coolant pressure $(\mathrm{MPa})$ & 15.5 & 15.5 & 15.5 \\
\hline
\end{tabular}

of the cladding reducing the gap thickness resulting in increased heat transfer. Contact between the fuel and cladding is observed at the times where the slope in the temperature curves changes significantly. While $\mathrm{U}_{3} \mathrm{Si}_{2}$ has a larger thermal expansion coefficient compared to the $\mathrm{UO}_{2}$, the inclusion of a relocation model in $\mathrm{UO}_{2}$ results in contact being established slightly earlier in the $\mathrm{UO}_{2} /$ Zircaloy-4 rodlet compared to the $\mathrm{U}_{3} \mathrm{Si}_{2} / \mathrm{Zircaloy}-4$. Due to the limited creep experienced by $\mathrm{FeCrAl}$, the gap remains open significantly longer and contact is established near the end of the irradiation.

The time evolution of hoop strain and stress at the inner surface of the cladding is plotted in Figure 6.2. Once again the change in slope of the curves correspond to the onset of contact. The initial non-zero strain is entirely driven by thermal expansion with $\mathrm{FeCrAl}$ having a larger thermal expansion than Zircaloy-4. Both the $\mathrm{UO}_{2} /$ Zircaloy- 4 and $\mathrm{U}_{3} \mathrm{Si}_{2} / \mathrm{Zircaloy}-4$ rodlets experience significant decreases in the hoop strain up to the point of contact due to the large creep rate in Zircaloy-4. The change in slope is less drastic in the $\mathrm{UO}_{2}$ fueled rods because creep is taken into account and the fuel is more compliant with the cladding. Rapid increases in strains is observed in the $\mathrm{U}_{3} \mathrm{Si}_{2} /$ Zircaloy-4 rodlet because no creep model exists for $\mathrm{U}_{3} \mathrm{Si}_{2}$, and it is thus treated as elastic. In addition, the swelling strains are significantly larger resulting in harder contact with the cladding occurring later in the irradiation history.

As for the hoop stress at the inner surface shown in Figure 6.2b, the magnitude of the stress is larger (compressive) initially in the $\mathrm{UO}_{2} / \mathrm{FeCrAl}$ due to the reduced cladding thickness. Upon contact both the $\mathrm{UO}_{2} / \mathrm{FeCrAl}$ and $\mathrm{U}_{3} \mathrm{Si}_{2} / \mathrm{Zircaloy}-4$ rods experience larger changes in hoop stress 


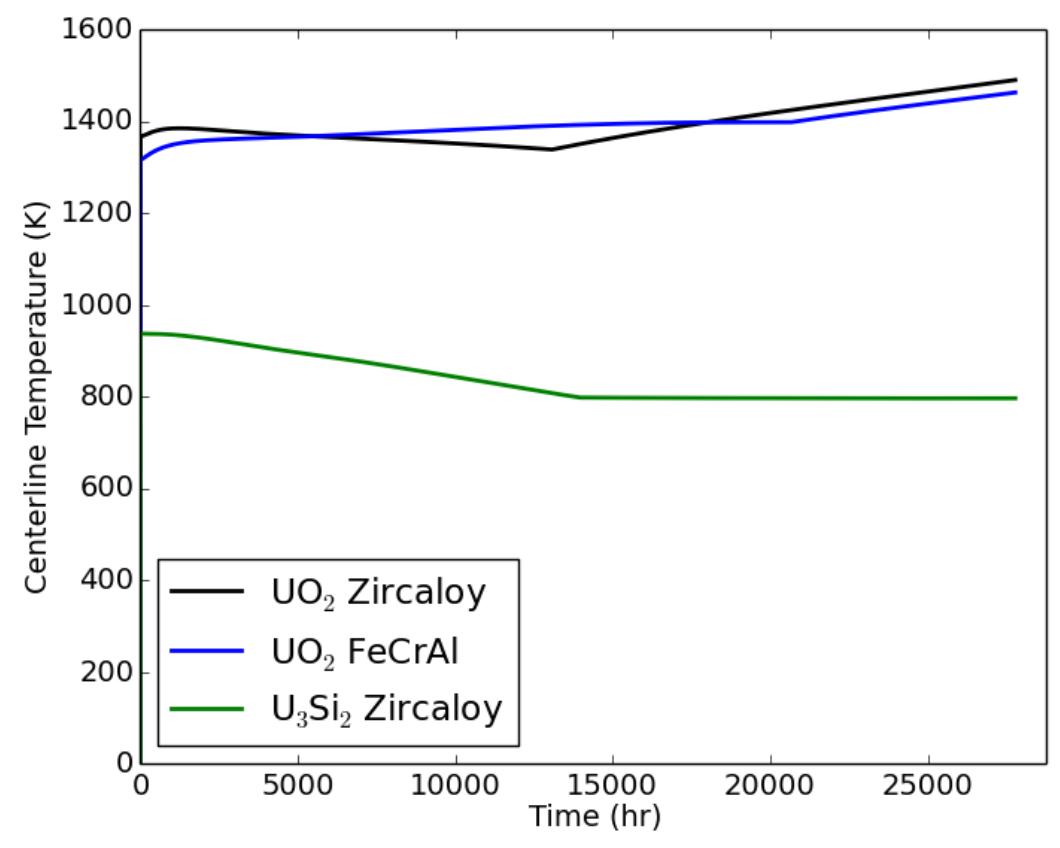

Figure 6.1: Centerline temperature evolution during normal operation for the three rodlets.

compared to the $\mathrm{UO}_{2} / \mathrm{Zircaloy}-4$ case. The cause in the $\mathrm{UO}_{2} / \mathrm{FeCrAl}$ rodlet is driven by the reduced cladding thickness for $\mathrm{FeCrAl}$ whereas in the $\mathrm{U}_{3} \mathrm{Si}_{2} / \mathrm{Zircaloy}-4$ case stress increases are driven by the elastic treatment of the fuel and the significantly larger gaseous swelling experienced in $\mathrm{U}_{3} \mathrm{Si}_{2}$.

Similar behavior is observed at the outer surface of the cladding in terms of hoop strain and stress evolution as illustrated in Figure 6.3. However, the magnitudes are slightly higher than at the inner surface as expected due it being at a larger radius.

Comparisons of the fission gas release as a function of irradiation time is shown in Figure 6.4 It is observed that fission gas begins to release from the fuel in all rodlets around the same time. However, the rate at which it is released in the $\mathrm{U}_{3} \mathrm{Si}_{2} /$ Zircaloy-4 rodlet is significantly higher than the $\mathrm{UO}_{2}$ fueled rodlets. This is expected because the diffusion coefficients for fission gases are larger in $\mathrm{U}_{3} \mathrm{Si}_{2}$ than in $\mathrm{UO}_{2}$. While the volume of fission gas released is constantly increasing, the relative fraction of gas released to that produced appears to decrease in the $\mathrm{U}_{3} \mathrm{Si}_{2} / \mathrm{Zircaloy}$ 4 rodlet. The cause for this is unknown and the lower length scale model is currently being revisited to understand the cause of this behavior and to determine whether or not it is physical. The main conclusion of the fission gas release plot is to highlight the fact that even though the diffusion coefficients are larger in $\mathrm{U}_{3} \mathrm{Si}_{2}$ than $\mathrm{UO}_{2}$, the fuel operates at a lower temperature in the $\mathrm{U}_{3} \mathrm{Si}_{2} /$ Zircaloy-4 resulting in comparable fission gas release magnitudes in all rodlets.

The evolution of the internal rod pressure is shown in Figure 6.5. As expected, the $\mathrm{UO}_{2} / \mathrm{FeCrAl}$ 


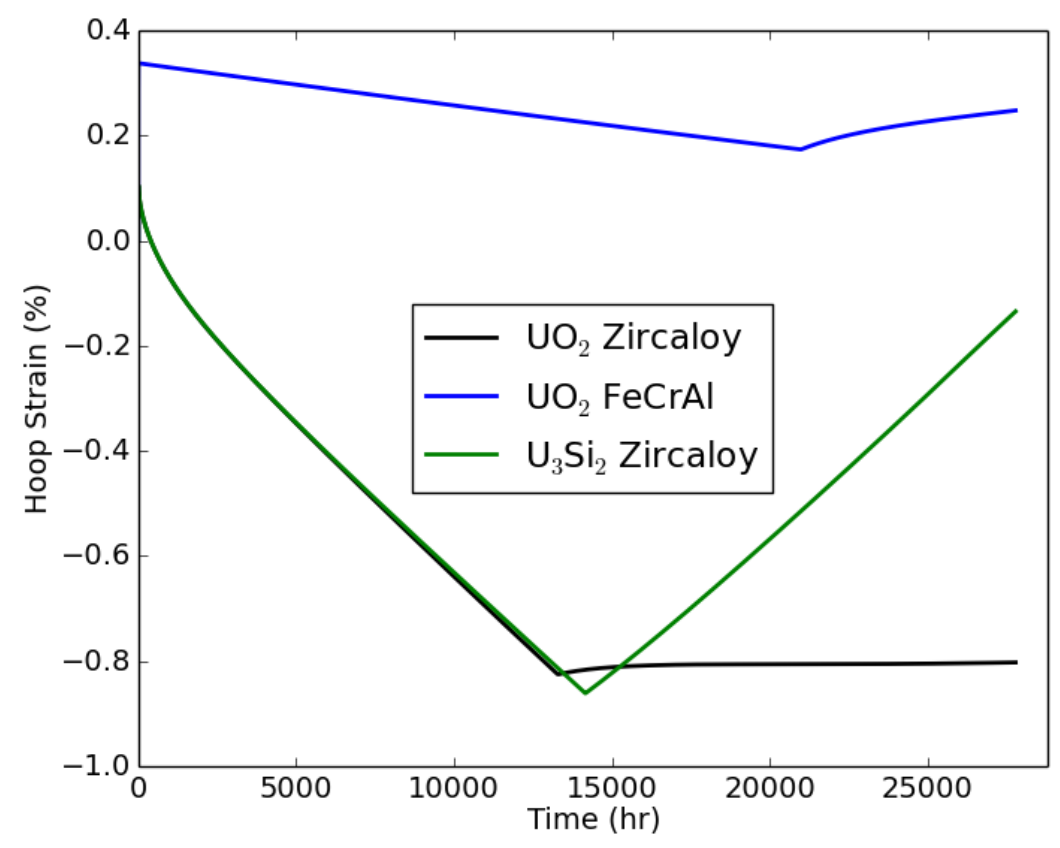

(a)

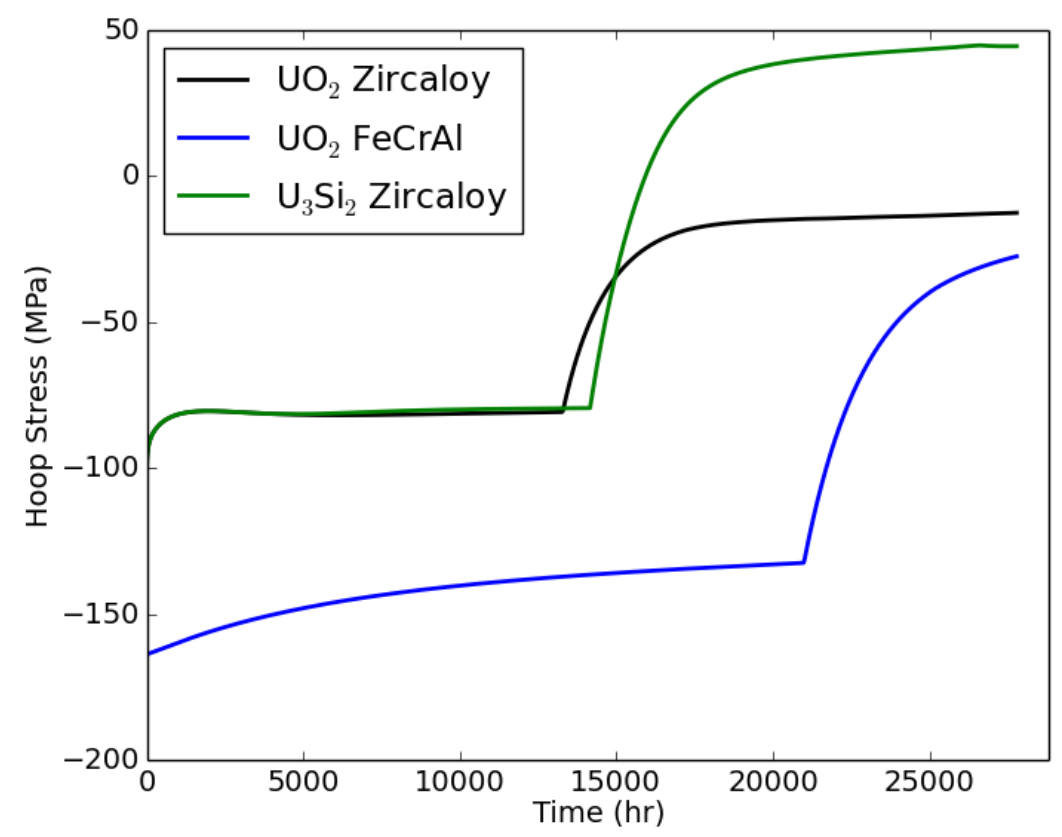

(b)

Figure 6.2: (a) hoop strain and (b) hoop stress evolution at the cladding inner surface at an axial position of $\sim 60 \mathrm{~mm}$ during normal operation for the three rodlets. 


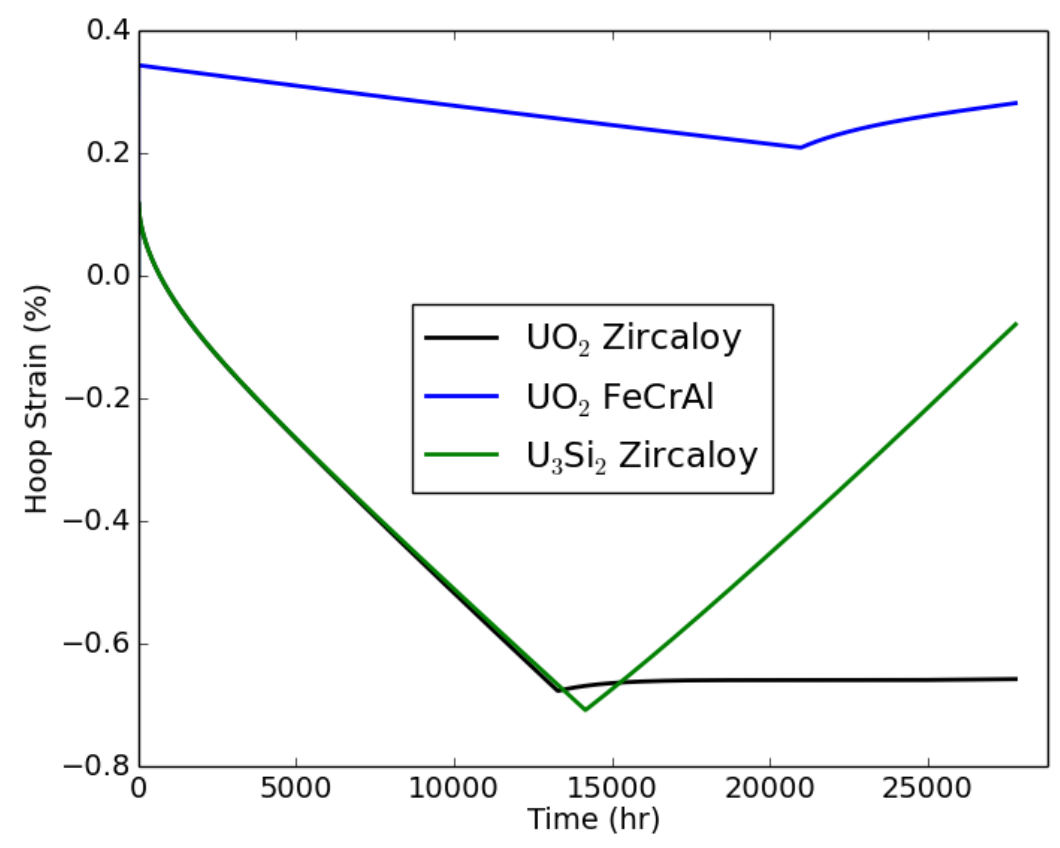

(a)

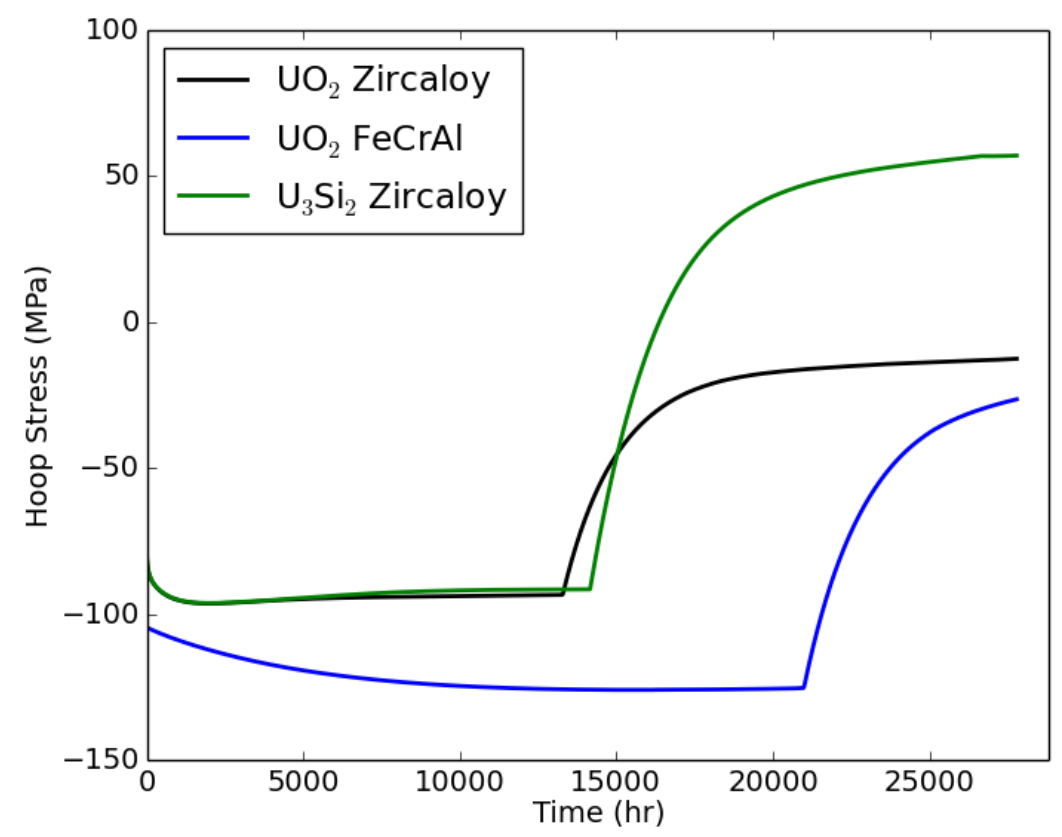

(b)

Figure 6.3: (a) hoop strain and (b) hoop stress evolution at the cladding outer surface at an axial position of $\sim 60 \mathrm{~mm}$ during normal operation for the three rodlets. 


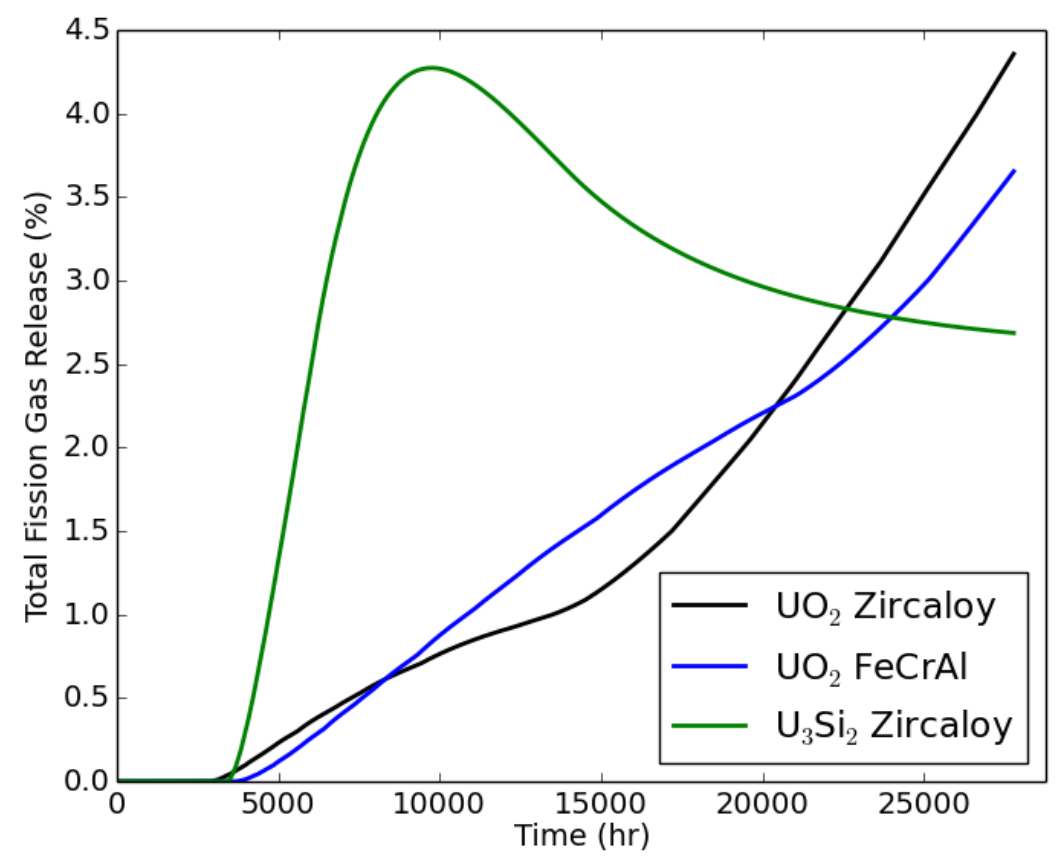

Figure 6.4: Fission gas release evolution during normal operation for the three rodlets.

rodlet experiences lower rod internal pressures for the duration of irradiation because of the increased gap thickness due to the reduced creep rates of FeCrAl. By the end of the 3.2 year irradiation the internal pressure is similar in all rods.

The last fuel performance parameter of interest in the normal operation comparisons is the oxide thickness illustrated in Figure 6.6. The oxide thickness evolution is very similar in all the rods up to about 7000 hours at which point the Zircaloy-4 cladded rods experience breakaway oxidation resulting in a transition from parabolic oxide thickness growth to linear. $\mathrm{FeCrAl}$ is not expected to experience breakaway oxidation and the oxide growth model captures this behavior. For this irradiation time Zircaloy- 4 experiences the growth of an oxide that is about 7 times that of FeCrAl. This result confirms the anticipated improved oxidation kinetics of FeCrAl.

Keeping in mind the definition by Bragg-sitton et al. [1] of what characteristics a material requires to be considered accident tolerant, the results shown for normal operating conditions indicate that both the $\mathrm{U}_{3} \mathrm{Si}_{2} / \mathrm{Zircaloy}-4$ and $\mathrm{UO}_{2} / \mathrm{FeCrAl}$ rodlets show similar performance to the $\mathrm{UO}_{2} /$ Zircaloy-4 with the exception of improved fuel centerline temperatures and oxide growth behavior, respectively. 


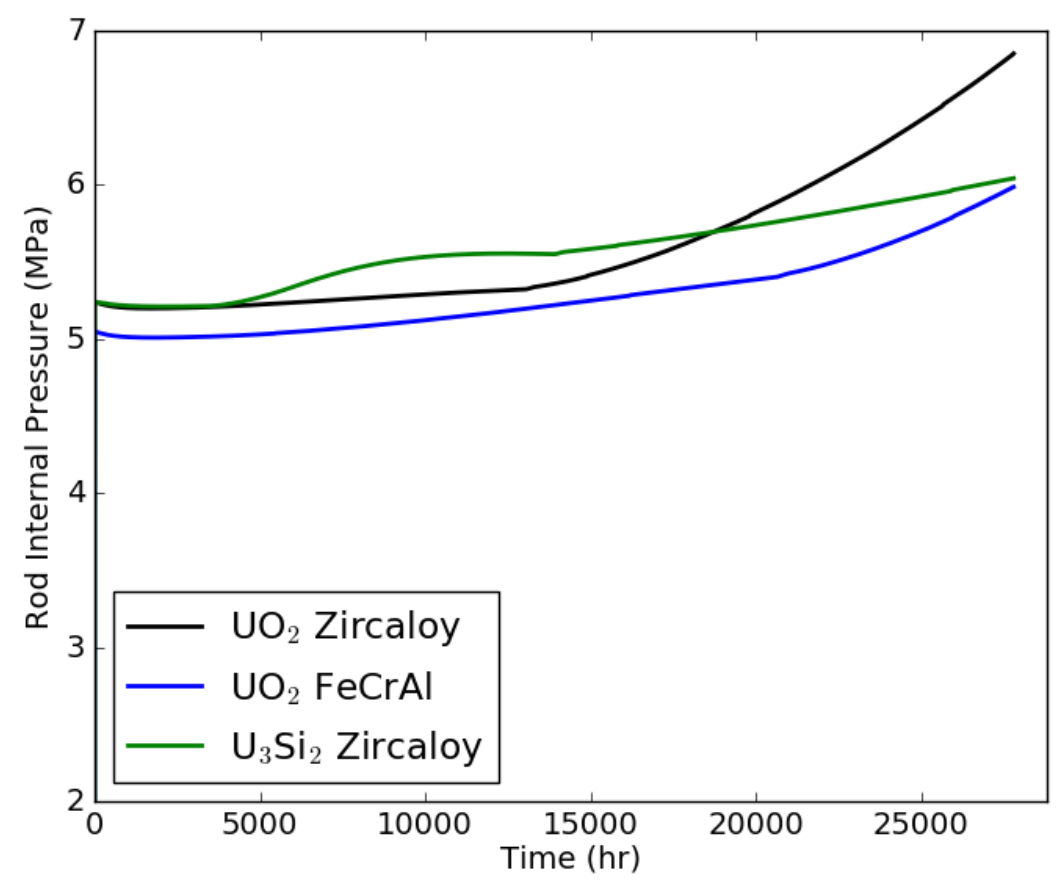

Figure 6.5: Rod internal pressure evolution during normal operation for the three rodlets.

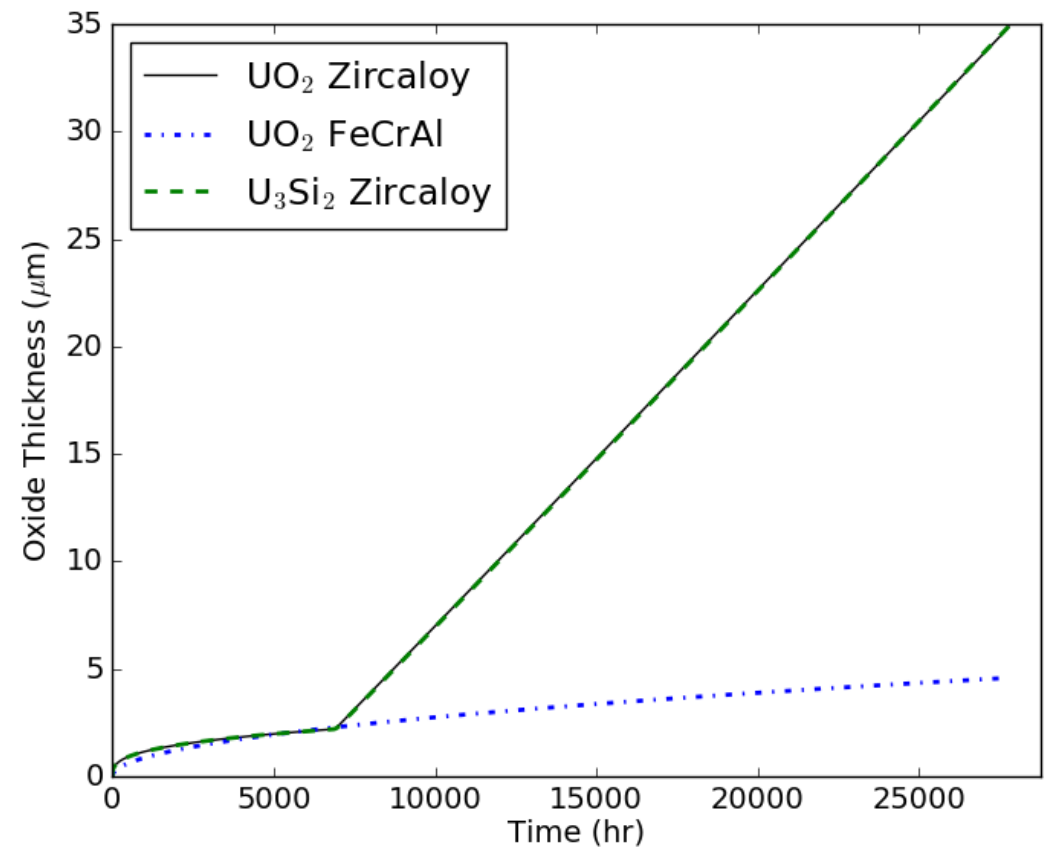

Figure 6.6: Maximum oxide thickness evolution during normal operation for the three rodlets. 


\subsubsection{Sensitivity Analysis}

Even as the three year ATF HIP draws to a close and lower length scale models have been developed, experimental data is still lacking and uncertainty exists in the currently available models. Therefore to assess the importance of this uncertainty in some of the parameters, sensitivity analyses have been completed for the normal operating case $\mathrm{U}_{3} \mathrm{Si}_{2} / \mathrm{Zircaloy}-4$ and $\mathrm{UO}_{2} / \mathrm{FeCrAl}$ rodlets. There are numerous statistical methodologies available to determine the sensitivity of the output metrics of interest to uncertainties in select input parameters including Pearson and Spearman correlation coefficients, main effects studies, surrogate models, and variance based decomposition. In this work, BISON is coupled to the DAKOTA [60] sensitivity analysis software to perform main effects studies.

\subsubsection{1 $\mathrm{UO}_{2} / \mathrm{FeCrAI}$ Rodlet}

The variables whose uncertainties are of interest in assessing their effect on fuel performance metrics vary depending upon the type of simulation being considered (normal operation or transient). For the $\mathrm{UO}_{2} / \mathrm{FeCrAl}$ rodlet during normal operation only three parameters were considered, listed in Table 6.2, which included a scaling factor on the Young's modulus, oxidation rate constant, and the thermal creep pre-exponential coefficient. In a main effects study a histogram distribution is used such that distinct values of each input parameter chosen and every combination is used in a separate simulation. Therefore varying a large selection of input parameters becomes prohibitive computationally, with a maximum of five input parameters recommended. In the table the three values for each parameter are denoted minimum, intermediate, and maximum. The intermediate value need not be the mean value as is the case with the thermal creep scale factor for which scaling by orders of magnitude was deemed appropriate due to the significant uncertainty associated with the thermal creep behavior of $\mathrm{FeCrAl}$ alloys .

Table 6.2: Parameters varied in the normal operation main effects for the $\mathrm{UO}_{2} / \mathrm{FeCrAl}$ rodlet.

\begin{tabular}{lccc}
\hline & Minimum & Intermediate & Maximum \\
\hline Young's modulus scale factor & 0.9 & 1.0 & 1.1 \\
Oxidation scale factor & 0.8 & 1.0 & 1.2 \\
Thermal creep scale factor & 1.0 & 10.0 & 100.0 \\
\hline
\end{tabular}

Figures 6.7 to 6.10 illustrate the main effects plots for the $\mathrm{UO}_{2} / \mathrm{FeCrAl}$ rodlet during normal operation. In a main effects study the output metric of interest (e.g., centerline temperature) is shown on the ordinate axis, whereas the various input parameters are plotted along the abscissa. Each subplot in a particular diagram represents the main effects of that input parameter. To provide guidance on how to interpret the results of a main effects study initially focus on the thermal creep scale factor subplot in Figure 6.7. The point for a thermal creep scale factor of 10 represents the mean value for centerline temperature obtained from all of the simulations that had the thermal creep scale factor set to 0.8. A monotonic upward slope when an input 
variable is varied indicates a positive correlation with the output metric. Correspondingly, a monotonic downward slope represents a negative correlation between the input parameter and output metric. Lines that are close to horizontal imply minute or no correlation between input and output. Note that all of the data points represent the mean values at the last timestep of the simulation.

Based upon this description on how to interpret the results presented in the main effects plots it is observed that for the centerline temperature at the end of the simulation only the thermal creep scaling factor has any influence on the mean centerline temperature obtained. As the scaling factor is increased the centerline temperature is reduced slightly. This is expected because contact will be established slightly earlier the more the cladding creeps resulting in slightly lower temperatures. The reader should examine the ordinate and notice that the range of centerline temperatures from all simulations is very small $(\sim 2.5 \mathrm{~K})$. While the temperature rise across the oxide scale thickness is taken into account, the rise is extremely small over such a thin oxide layer that the centerline temperature is not impacted.
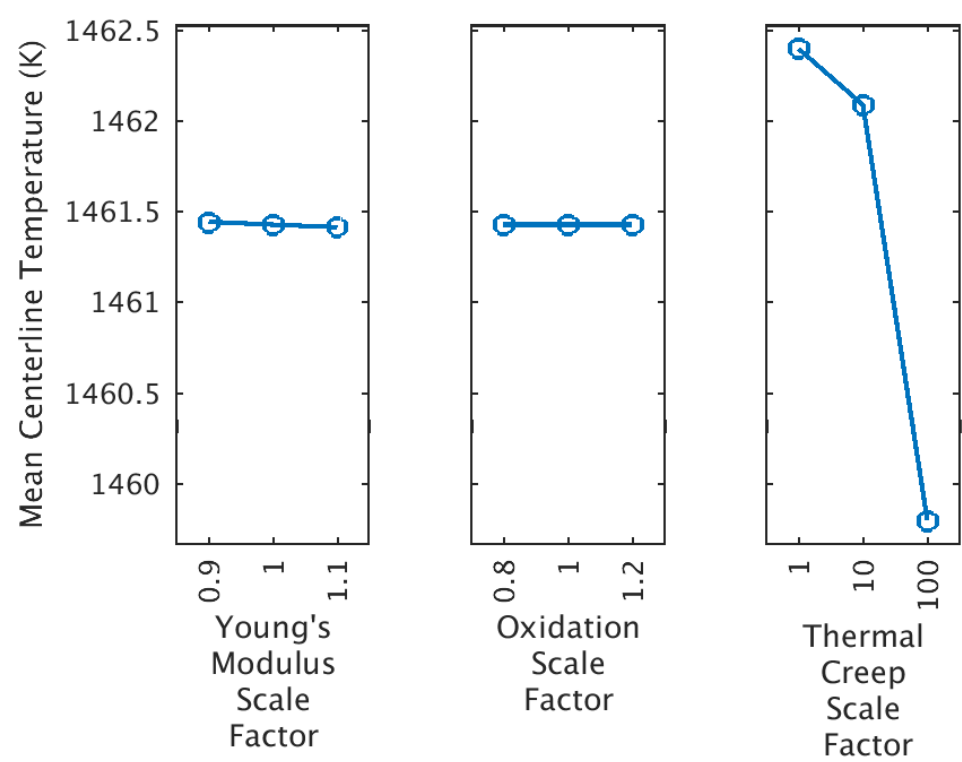

Figure 6.7: Main effects plot for centerline temperature at the end of the normal operation simulation for the $\mathrm{UO}_{2} / \mathrm{FeCrAl}$ rodlet.

Typically, the cladding in nuclear fuel rods is treated as a thin-walled cylinder when analyzing the mechanical behavior due to their small thicknesses. Based upon this assumption it would be expected to see similar trends in the sensitivity analysis at the inner and outer surfaces. However, Figures 6.8 and 6.9 illustrate different sensitivity behavior at the inner and outer surfaces. An examination of these results indicate that the mean hoop strain is fairly independent of the variation in the input parameters at both the inner and outer surfaces where as the mean hoop stress is influenced by the Young's modulus and thermal creep scale factors at the inner and outer 
surfaces. In both cases the stresses are still compressive at the end of the simulation. At the inner surface the Young's modulus of the cladding has almost no correlation with the hoop stress whereas the thermal creep scaling factor has quite a strong positive correlation with the hoop stress (increasing the creep rate reduces the magnitude of the compressive stress). Increasing the thermal creep scaling factor to 10 results in the stress becoming more compressive before becoming less compressive in the cases with a scaling factor 100. Once again it should be noted that range of stresses is fairly small in both cases. The oxidation scaling factor has no influence on the mechanical behavior because Bison currently does not couple the oxide thickness formation to the reduction in strength of the underlying substrate (in this case $\mathrm{FeCrAl}$ ).

The last fuel performance parameter considered in the normal operating sensitivity analysis for the $\mathrm{UO}_{2} / \mathrm{FeCrAl}$ rodlet is the fission gas release shown in Figure 6.10. Examination of this figure shows similar behavior as the fuel centerline temperature. The oxidation scale and thermal creep scale factors show the same trends with the slight monotonic decrease in the Young's modulus scale factor changing to a slight monotonic increase.

In summary the sensitivity analysis during normal operation for the $\mathrm{UO}_{2} / \mathrm{FeCrAl}$ rodlet illustrates that while some of the uncertain parameters have an influence on the temperature, hoop stresses and strains, and fission gas release, the effects are minimal based upon the small ranges observed on all the ordinate axes.

\subsubsection{2 $\mathrm{U}_{3} \mathrm{Si}_{2} /$ Zircaloy-4 Rodlet}

Given the uncertainty associated with the recently added $\mathrm{U}_{3} \mathrm{Si}_{2}$ fission gas release model, the number of uncertain parameters used in the sensitivity analysis for the $\mathrm{U}_{3} \mathrm{Si}_{2} /$ Zircaloy-4 was five, including three fission gas parameters, the power, and the thermal conductivity. Varying these parameters exercises two of the lower length scale models added to BISON for $\mathrm{U}_{3} \mathrm{Si}_{2}$ fuel performance, namely the thermal conductivity degradation model and the fission gas release model. In the table IG and GB represent intergranular and grain boundary, respectively. Five uncertain input parameters result in 243 cases being included in the main effects analysis. The same output parameters as for the $\mathrm{UO}_{2} / \mathrm{FeCrAl}$ main effects analysis were used here.

Table 6.3: Parameters varied in the normal operation main effects for the $\mathrm{U}_{3} \mathrm{Si}_{2} / \mathrm{Zircaloy}^{4}$ rodlet.

\begin{tabular}{lccc}
\hline & Minimum & Intermediate & Maximum \\
\hline Resolution parameter scale factor & 0.1 & 1.0 & 10.0 \\
IG Diffusion Coefficient scale factor & 0.1 & 1.0 & 10.0 \\
GB Diffusion Coefficient scale factor & 0.1 & 1.0 & 10.0 \\
Power scale factor & 0.9 & 1.0 & 1.1 \\
Thermal conductivity scale factor & 0.9 & 1.0 & 1.1 \\
\hline
\end{tabular}



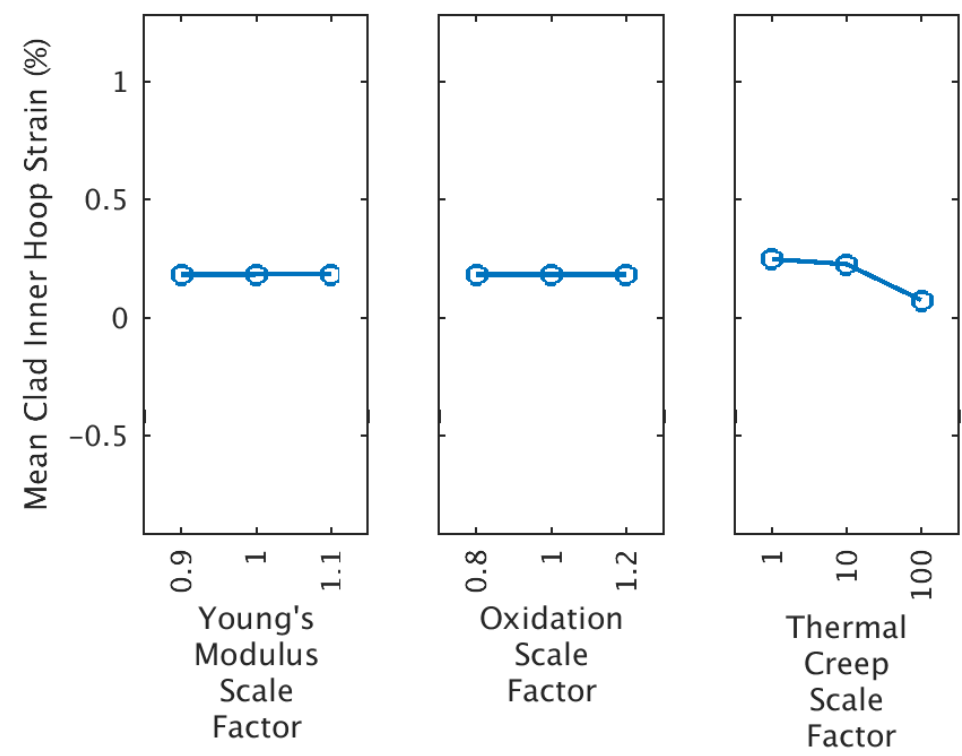

(a)
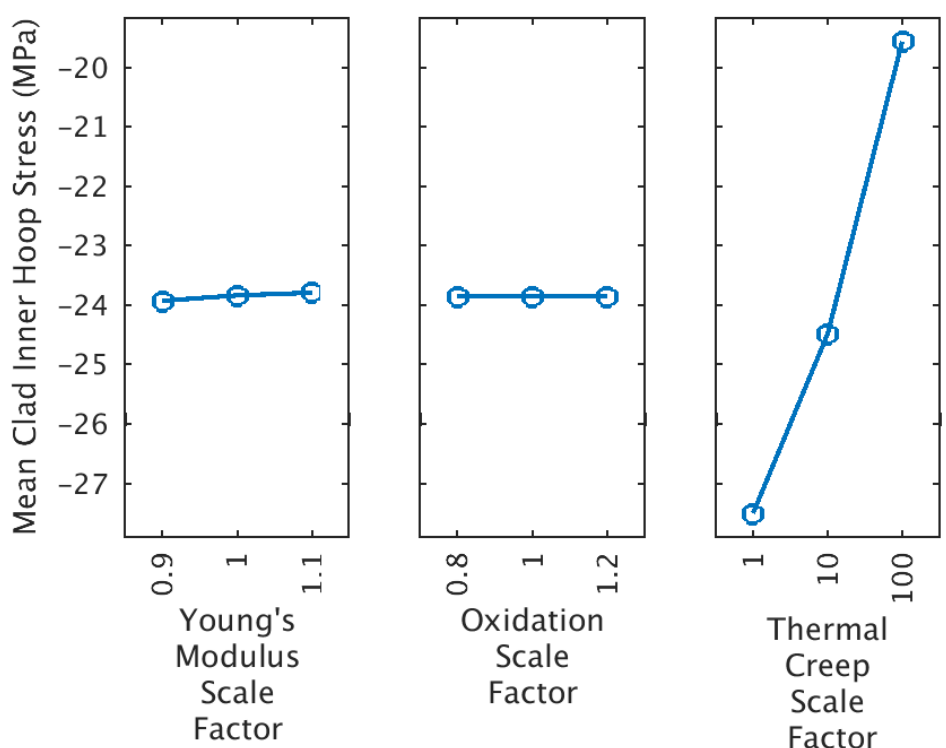

(b)

Figure 6.8: Main effects plots for (a) hoop strain and (b) hoop stress at the inner surface of the cladding at the end of the normal operation simulation for the $\mathrm{UO}_{2} / \mathrm{FeCrAl}$ rodlet.

Figures 6.11 through 6.14 illustrate the results of the main effects study for the $\mathrm{U}_{3} \mathrm{Si}_{2} / \mathrm{Zircaloy}-4$ rodlet during normal operation. In contrast to the $\mathrm{UO}_{2} / \mathrm{FeCrAl}$ rodlet results presented earlier the 

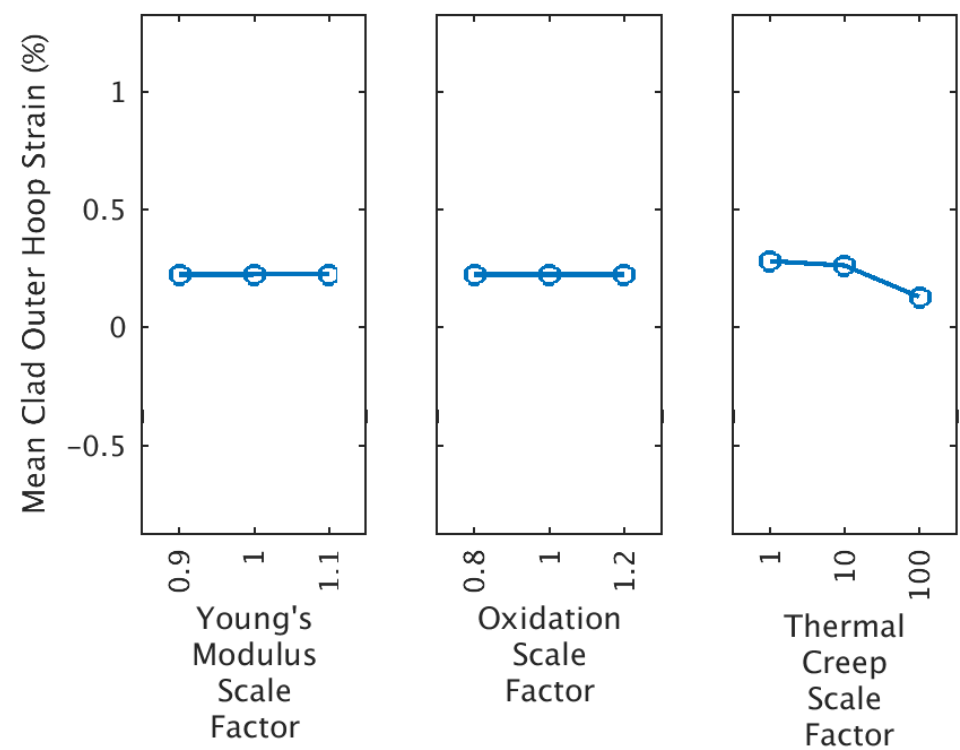

(a)
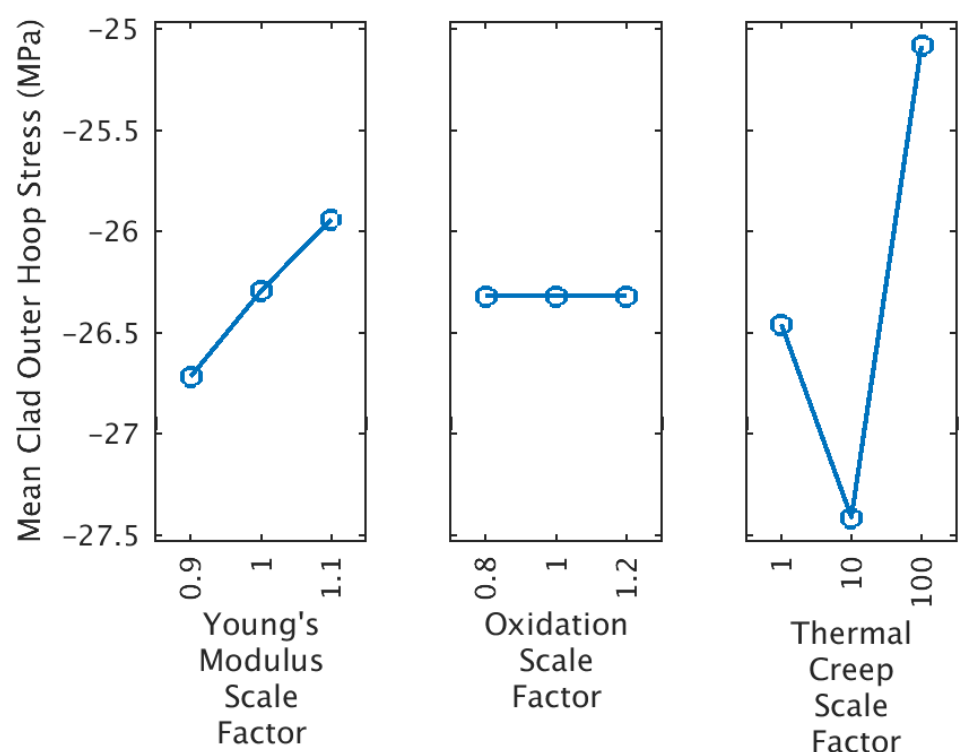

(b)

Figure 6.9: Main effects plots for (a) hoop strain and (b) hoop stress at the outer surface of the cladding at the end of the normal operation simulation for the $\mathrm{UO}_{2} / \mathrm{FeCrAl}$ rodlet.

ranges on some of the output parameters of interest are larger. This indicates that the uncertainty in the $\mathrm{U}_{3} \mathrm{Si}_{2}$ models and parameters are more severe. 

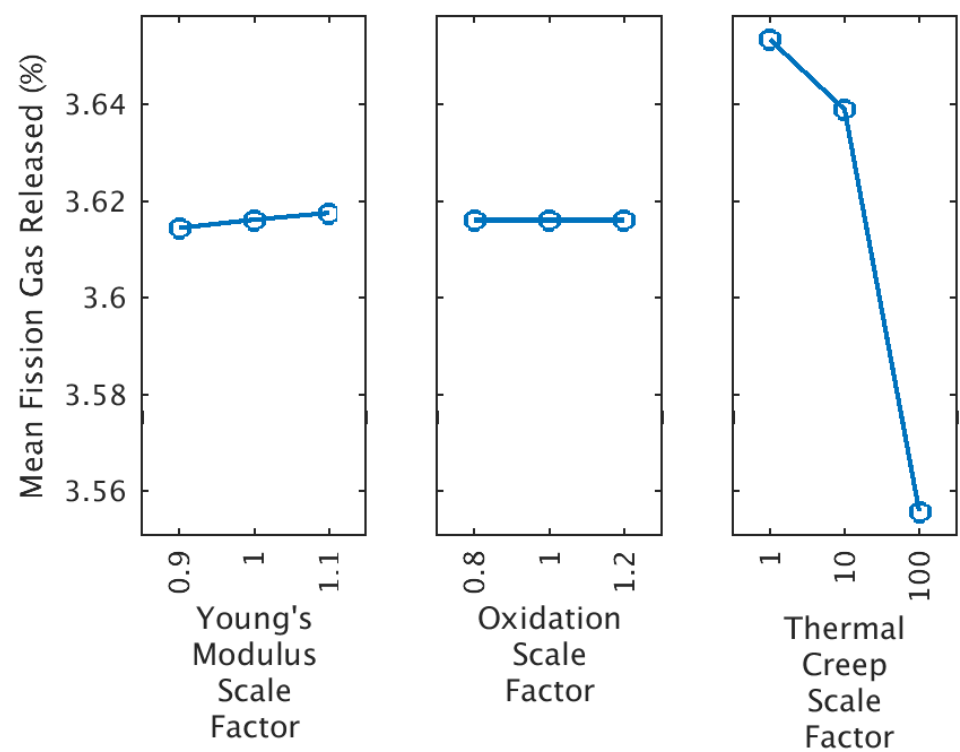

Figure 6.10: Main effects plot for fission gas release at the end of the normal operation simulation for the $\mathrm{UO}_{2} / \mathrm{FeCrAl}$ rodlet.

As expected the scaling of the power to the fuel has a strong positive correlation with the centerline temperature (i.e., as the power is increased the temperature rises). On the other hand, as the thermal conductivity is increased the centerline temperature decreases. The three fission gas parameters appear to have a minimal influence on the centerline temperature results; however, one will notices small but positive correlation to the temperature for all three parameters.

Figure 6.12 presents the hoop strain and stress main effects analysis at the inner surface of the cladding. In the case of hoop strain, the dominate parameter was the power scaling factor whereas in the case of the hoop stress the most influential parameter is the thermal conductivity scaling factor of the fuel. The stress and strain are larger when the power is high or the thermal conductivity low due to increased swelling and temperatures. Figure 6.13 shows the main effects analysis at the outer surface of the cladding. Here, the trends are similar to the inner surface except the magnitudes of the stresses and strains being larger as expected due to the slightly larger radius to the outer surface from the rod centerline.

The fission gas released main effects study results for normal operation are illustrated in Figure 6.14. These results are the most interesting of all the main effects plots analyzed for either of the rodlets during normal operation. There is significant uncertainty in the fission gas released model for $\mathrm{U}_{3} \mathrm{Si}_{2}$ considering it is developed based upon a single data point in the literature for irradiation at light water reactor temperatures. In fission gas release modeling, the behavior of the gases strongly depend on the resolution parameter and diffusion coefficients as confirmed by the main effects analysis. Each of the three fission gas parameters have a positive correlation with 

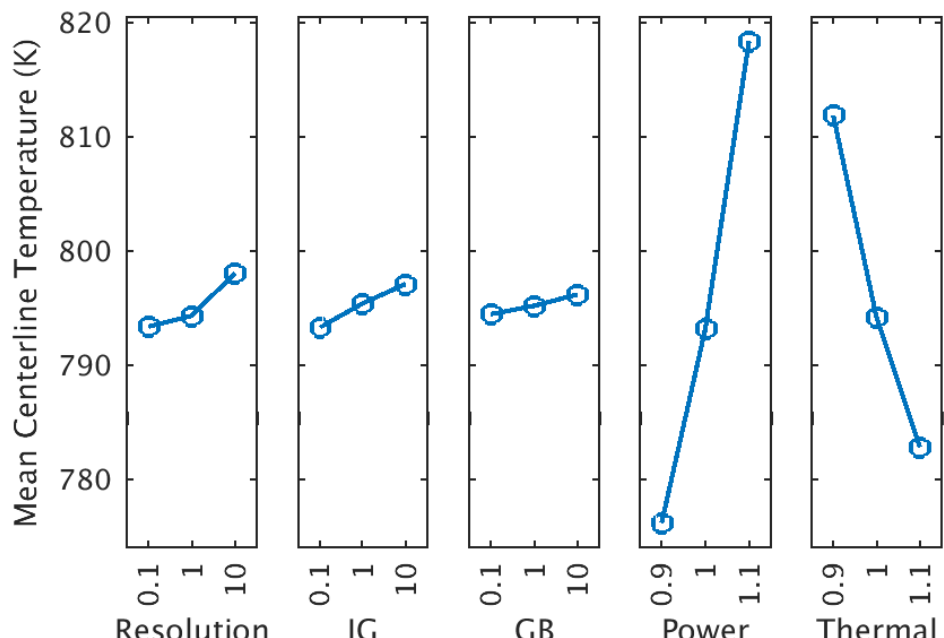
Parameter Diff. Coeff. Diff. Coeff.
Scale
Scale
Scale
Factor
Factor
Factor

Scale

Factor

Conductivity

Scale

Factor

Figure 6.11: Main effects plot for centerline temperature at the end of the normal operation simulation for the $\mathrm{U}_{3} \mathrm{Si}_{2} /$ Zircaloy-4 rodlet.

the amount of gas released with the resolution parameter having the greatest influence. Increasing the rate at which the gas can diffuse through the fuel will result in more gas being released. In addition, increasing the power results in more fission gas release because of more fissions and the fact that the temperature of the fuel is increased leading to faster diffusion through the Soret effect. Decreasing the thermal conductivity results in more fission gas being released due to higher temperatures. Based upon the large variation in fission gas release simply by scaling the fission gas behavior by one order of magnitude, the importance of conducting further experiments to help understand the fission gas behavior mechanisms in $\mathrm{U}_{3} \mathrm{Si}_{2}$ is clear.

\subsection{Loss of Coolant Accident}

\subsubsection{Nominal Case}

Materials being considered to be for use in light water reactors for enhanced accident tolerance must demonstrate improved performance under postulated accident conditions. To gain insight into relative behavior between the $\mathrm{UO}_{2} / \mathrm{Zirclaoy}-4, \mathrm{U}_{3} \mathrm{Si}_{2} / \mathrm{Zircaloy}-4$, and $\mathrm{UO}_{2} / \mathrm{FeCrAl}$ rodlets during these conditions, a postulated LOCA was appended to the end of the normal operation irradiation described earlier. The conditions for this LOCA were to be representative of a large break LOCA. The LOCA was simulated by dropping the power to the fuel over 2 seconds to 

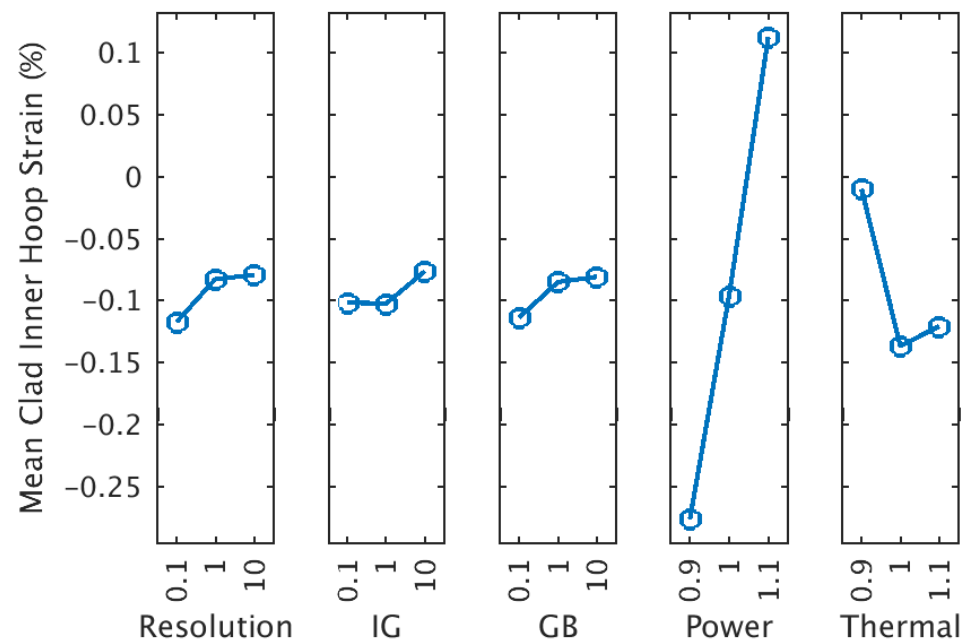

Resolution

Paramete

Diff. Coeff. Diff. Coeff.

Scale

Scale

Scale

Scale

Factor

Conductivity

Scale

Factor

(a)

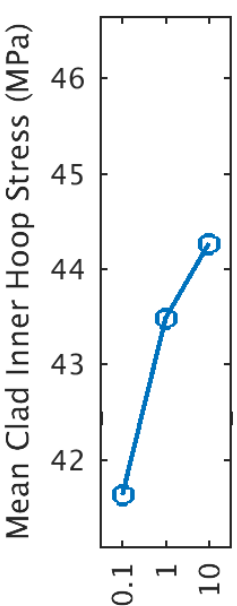

Resolution

Parameter Diff

Scale

Factor
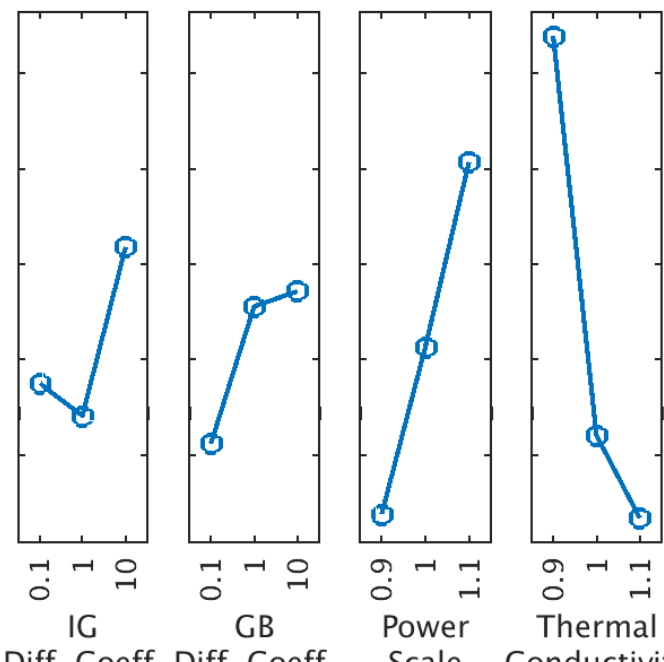

Scale Conductivity

Factor Scale

Factor

(b)

Figure 6.12: Main effects plots for (a) hoop strain and (b) hoop stress at the inner surface of the cladding at the end of the normal operation simulation for the $\mathrm{U}_{3} \mathrm{Si}_{2} /$ Zircaloy- 4 rodlet. 

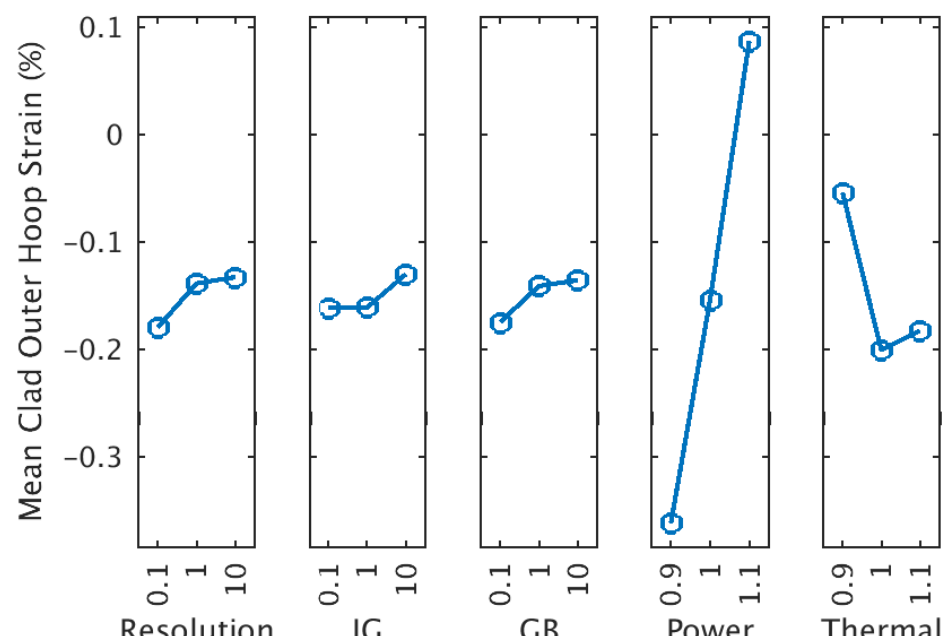

Resolution IG

GB

Parameter Diff. Coeff. Diff. Coeff.

Scale

Scale

Scale

Scale

Factor

Factor

Factor

Factor

๑)

Thermal

Conductivity

Scale

(a)
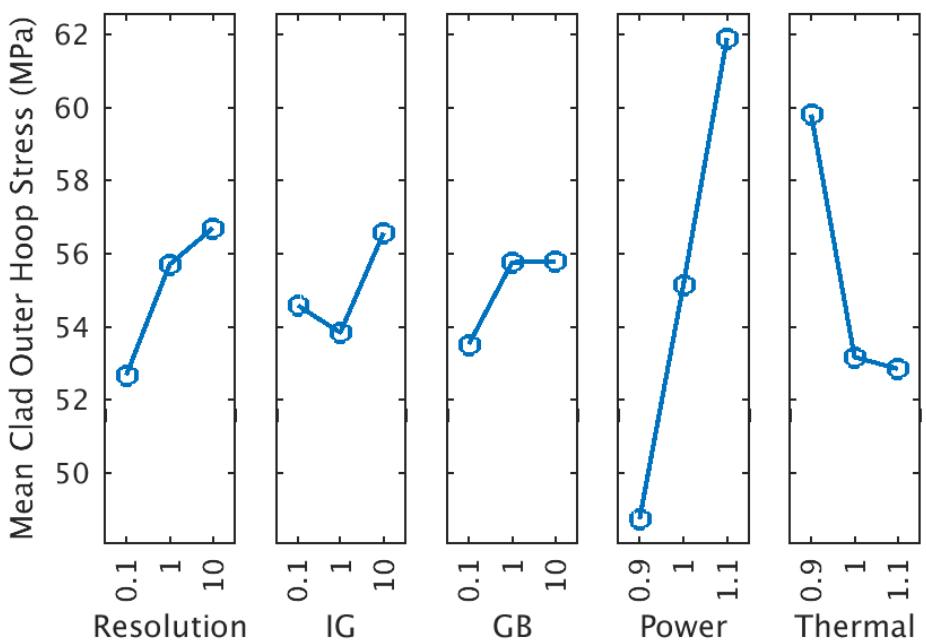

Resolution

IG

GB

Scale Scale Scale

Scale Conductivity

Factor Scale

Factor

Factor

Factor

Factor

(b)

Figure 6.13: Main effects plots for (a) hoop strain and (b) hoop stress at the outer surface of the cladding at the end of the normal operation simulation for the $\mathrm{U}_{3} \mathrm{Si}_{2} /$ Zircaloy- 4 rodlet. 


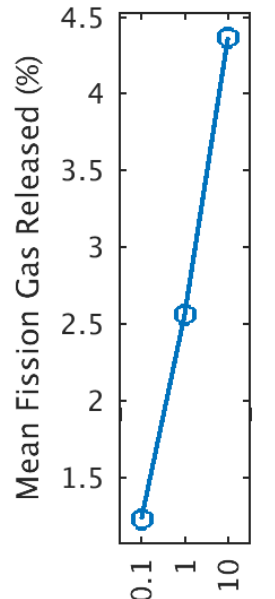

Resolution

Parameter

Scale

Factor

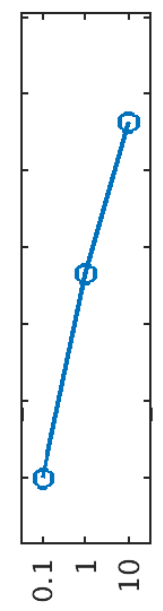

IG

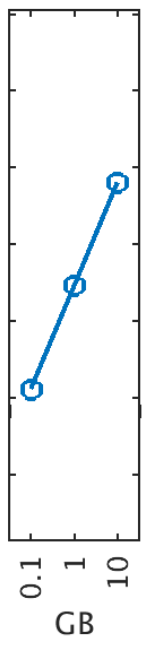

foeff.

Scale

Factor

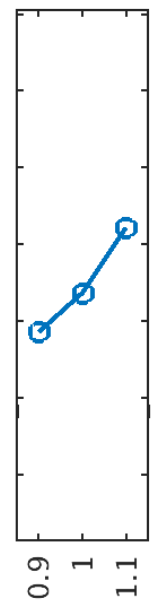

Power

Scale

Factor

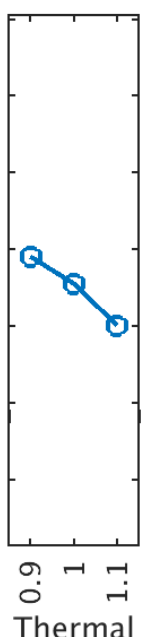

Conductivity

Scale

Factor

Figure 6.14: Main effects plot for fission gas release at the end of the normal operation simulation for the $\mathrm{U}_{3} \mathrm{Si}_{2} /$ Zircaloy-4 rodlet.

zero, turning on decay heat, and setting the inlet mass flux in the coolant channel model to 1 $\mathrm{kg} / \mathrm{m}^{2}$-s (zero cannot be specified as the subchannel coolant model would fail) over 10 seconds. The fuel performance parameters of interest at during the LOCA transient are the centerline temperature, hoop strain at the inner and outer surface of the cladding, and the rod internal pressure. In all the subsequent plots shown in this section the time scale indicates the beginning of the LOCA transient in seconds (i.e., the end of the base irradiation is set to $t=0$ ).

Figure 6.15 shows the fuel centerline temperature evolution during the LOCA transient. Initially the temperature is at its normal operation value as the power is still at $25 \mathrm{~kW} / \mathrm{m}$ at $\mathrm{t}=0$. After the power is shut off the temperature rapidly decreases followed by a continual increase in the temperature until cladding failure occurs. It should be noted that the failure criteria for the Zircaloy- 4 is taken as the plastic instability limit given by failure occurring if the creep rate exceeds $1 / 36 \mathrm{~s}^{-1}$. It is observed that both of the Zircaloy-4 tubes fail at a later time than FeCrAl indicated by the termination of the curve in time. The predicted time to cladding burst was $100.37,108,22$, and 118.28 seconds for the $\mathrm{UO}_{2} / \mathrm{FeCrAl}, \mathrm{U}_{3} \mathrm{Si}_{2} / \mathrm{Zircaloy}-4$, and $\mathrm{UO}_{2} /$ Zircaloy4 rodlets respectively.

The cladding hoop strain evolution in at both the inner and outer surfaces of the cladding during the LOCA transient are plotted in Figure 6.16. The trends are similar in both plots except that the strain attained on the outer surface is about $5 \%$ less than at the inner surface in the rodlets clad in Zircaloy-4 resulting in a thinning of the cladding during the ballooning process. While the $\mathrm{U}_{3} \mathrm{Si}_{2} /$ Zircaloy- 4 and $\mathrm{UO}_{2} /$ Zircaloy- 4 rodlets experience almost identical strains due 


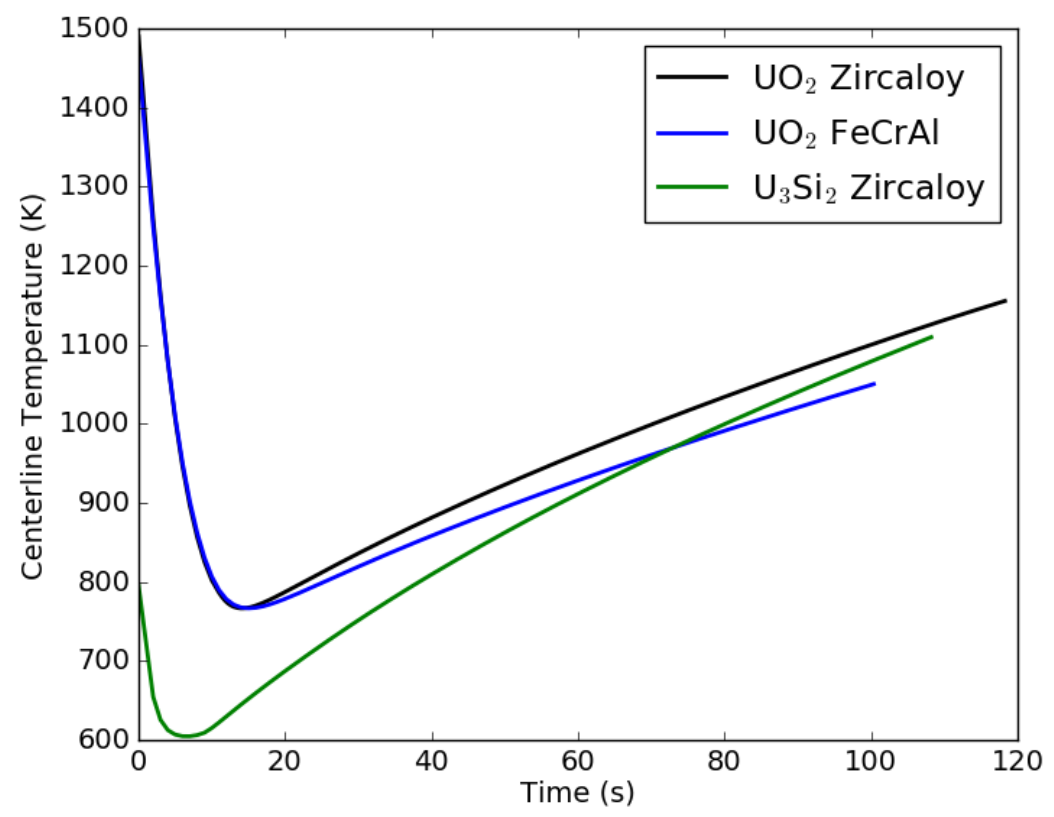

Figure 6.15: Centerline temperature evolution during the LOCA transient for the three rodlets.

to having the same cladding material, the $\mathrm{U}_{3} \mathrm{Si}_{2} /$ Zircaloy-4 rodlet fails earlier due to the larger pre-straining of the cladding during normal operation. This pre-straining is caused by the treatment of $\mathrm{U}_{3} \mathrm{Si}_{2}$ as elastic and the larger thermal expansion and gaseous swelling experienced by $\mathrm{U}_{3} \mathrm{Si}_{2}$. The larger pre-straining can be seen in Figures 6.2a and 6.3a. Despite having higher strength, the $\mathrm{UO}_{2} / \mathrm{FeCrAl}$ rodlet fails first due to having thinner cladding and lower creep rates. The absence of of high creep results in less ballooning and increases stresses causing failure earlier than the the Zircaloy-4 rodlets. However, the difference in burst time is only 20 seconds among the rodlets.

While $\mathrm{UO}_{2} / \mathrm{FeCrAl}$ rodlet fails earlier, its strain is significantly less than the Zircaloy-4 clad rodlets meaning that the ballooning is less severe. Constriction of the subchannels would be minor, which will allow coolability during reflooding to be maintained.

The time evolution of the pressure inside the rodlets during the LOCA transient is shown in Figure 6.17. As the fuel cools down during the power drop there is a slight decrease in the internal pressure of the rodlets. As the rod heats up due to the loss of coolant and heat transfer degradation at the cladding outer surface, the pressure initially begins to rise as the fuel expands and reduces the internal volume in the rod. Once ballooning of the cladding begins the pressure evolution is a competition between the increase in temperature and the increasing internal volume. Higher temperatures will cause the pressure to increase while a larger internal volume will cause the pressure to decrease. At a certain point when the cladding distention is sufficient, the pressure begins to drop continuously until failure. The larger pressure within the $\mathrm{UO}_{2} 2 / \mathrm{FeCrAl}$ rodlet at the time of failure will likely result in a much larger rupture opening as observed by 


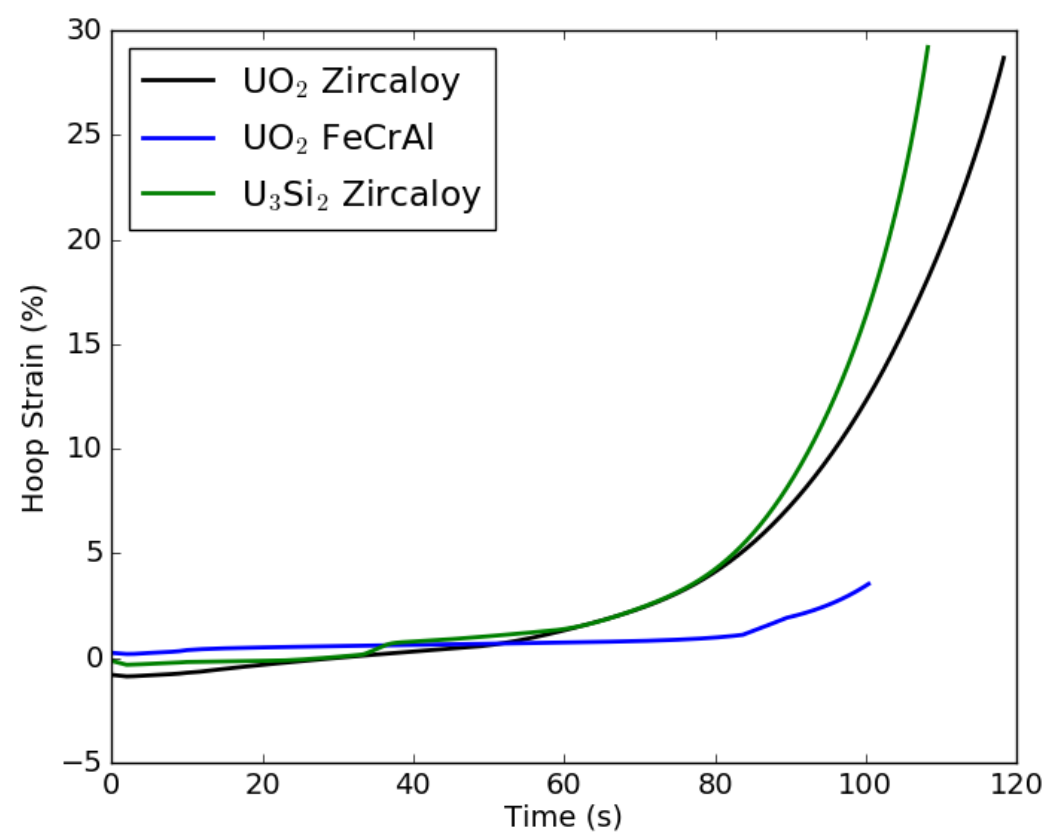

(a)

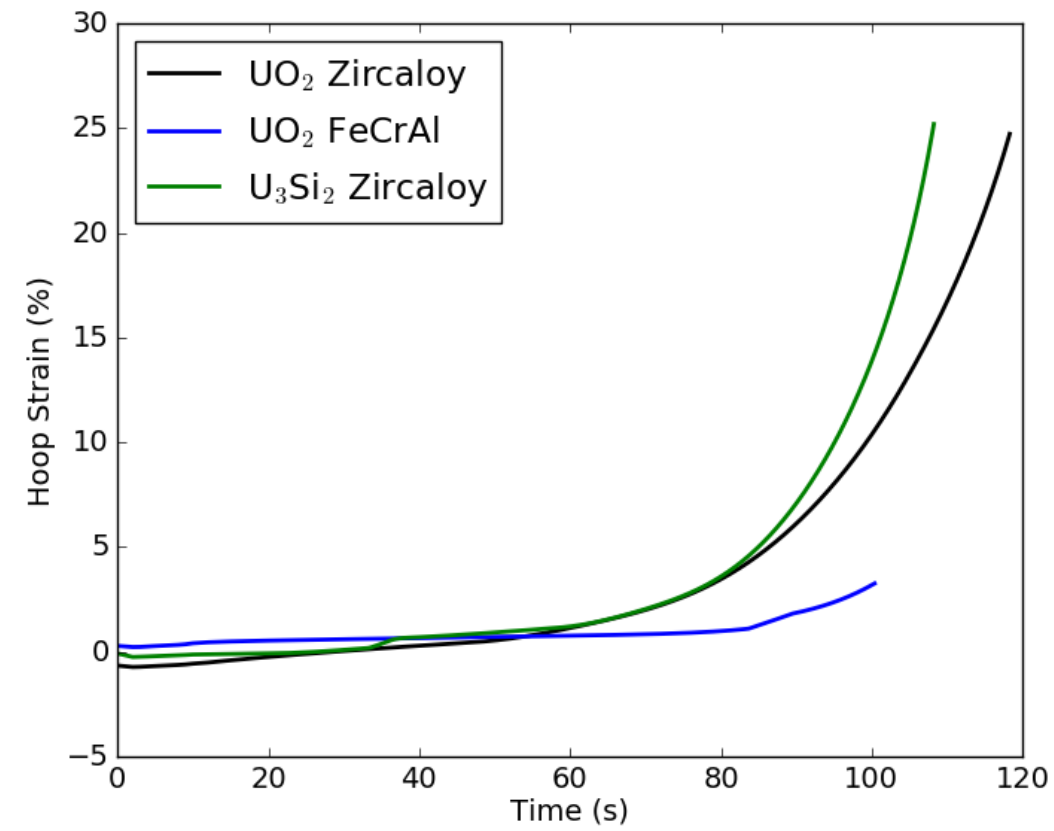

(b)

Figure 6.16: Evolution of the hoop strain at (a) the inner surface and (b) outer surface at an axial position of $\sim 60 \mathrm{~mm}$ during the LOCA transient for the three rodlets. 


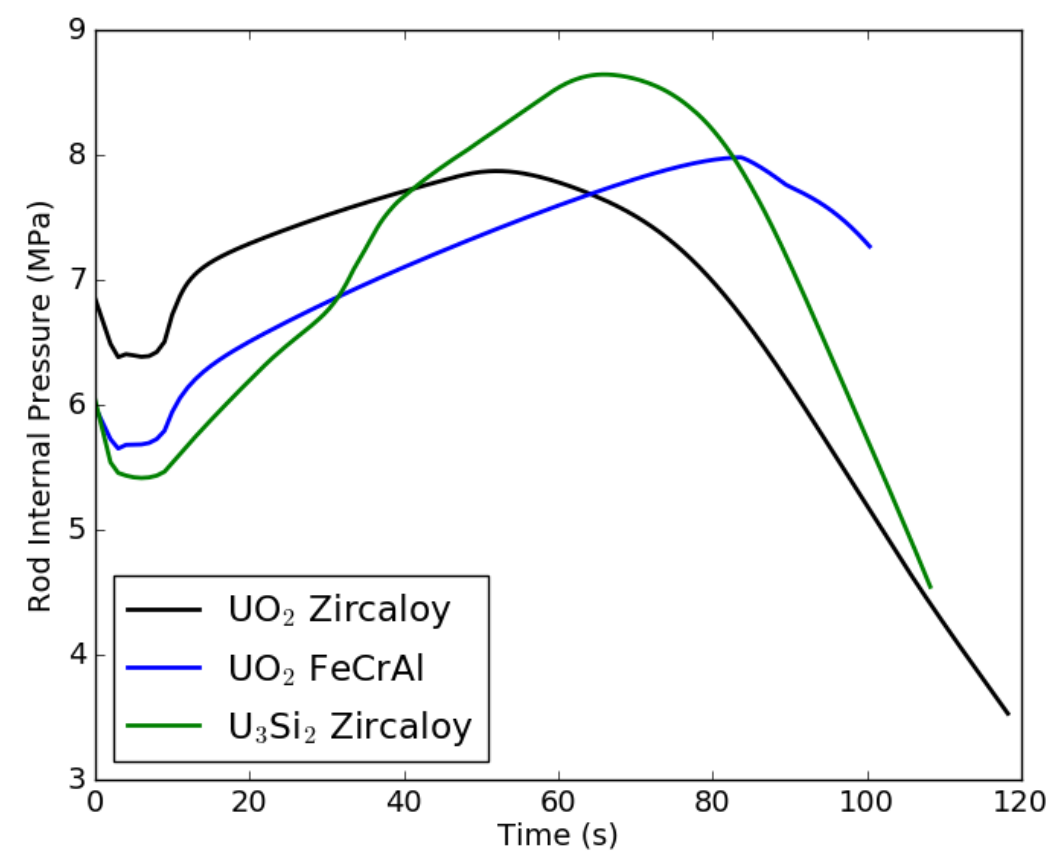

Figure 6.17: Rod internal pressure evolution during the LOCA transient for the three rodlets.

\subsubsection{Sensitivity Analysis}

As with the normal operation case, sensitivity analysis was completed for the LOCA scenario using main effects plots. In the LOCA case the output parameters of interest are the centerline temperature, hoop strain at the inner and outer cladding surface, and the time to cladding burst (failure). As before the main effects plots represent the mean values at the time of failure (end of the simulation) but it should be noted that the base irradiation is taken into account in the parameter variation.

\subsubsection{1 $\mathrm{UO}_{2} / \mathrm{FeCrAl}$ Rodlet}

For the $\mathrm{UO}_{2} / \mathrm{FeCrAl}$ rodlet the uncertain parameters for the LOCA transient are the same as the normal operation case with the addition of a scaling factor on the burst stress. The failure criterion developed by Gamble et al. [53] is based upon the only burst experiments completed on $\mathrm{FeCrAl}$ alloys. In these experiments there were only five $\mathrm{FeCrAl}$ rods, which is noted to be a small sampling of experiments. Therefore, a sensitivity analysis is conducted that takes into account this parameter to acknowledge the uncertainty that will associated with the burst stress correlation. 
Table 6.4: Parameters varied in the LOCA main effects for the $\mathrm{UO}_{2} / \mathrm{FeCrAl}$ rodlet.

\begin{tabular}{lccc}
\hline & Minimum & Intermediate & Maximum \\
\hline Young's modulus scale factor & 0.9 & 1.0 & 1.1 \\
Oxidation scale factor & 0.8 & 1.0 & 1.2 \\
Thermal creep scale factor & 1.0 & 10.0 & 100.0 \\
Burst stress scale factor & 0.8 & 1.0 & 1.2 \\
\hline
\end{tabular}

As expected the scaling of the burst stress is strongly correlated with the temperature. A lower burst stress means that the failure criterion will be met at a lower temperature since the burst stress is exponentially dependent on temperature. Correspondingly, a larger burst stress will mean the cladding will have to reach a higher temperature before failing. The thermal creep scaling factor has a unique correlation with the temperature. If increased by a single order of magnitude the higher creep rate results in a small amount of cladding ballooning to help reduce the internal pressure and stress within the cladding, resulting in failure at a higher temperature. However, if the thermal creep scale factor is increased by another order of magnitude the mean fuel centerline temperature reached is lower. Once again the oxidation scale factor has no correlation due to the temperature drop across the oxide thickness being extremely small.
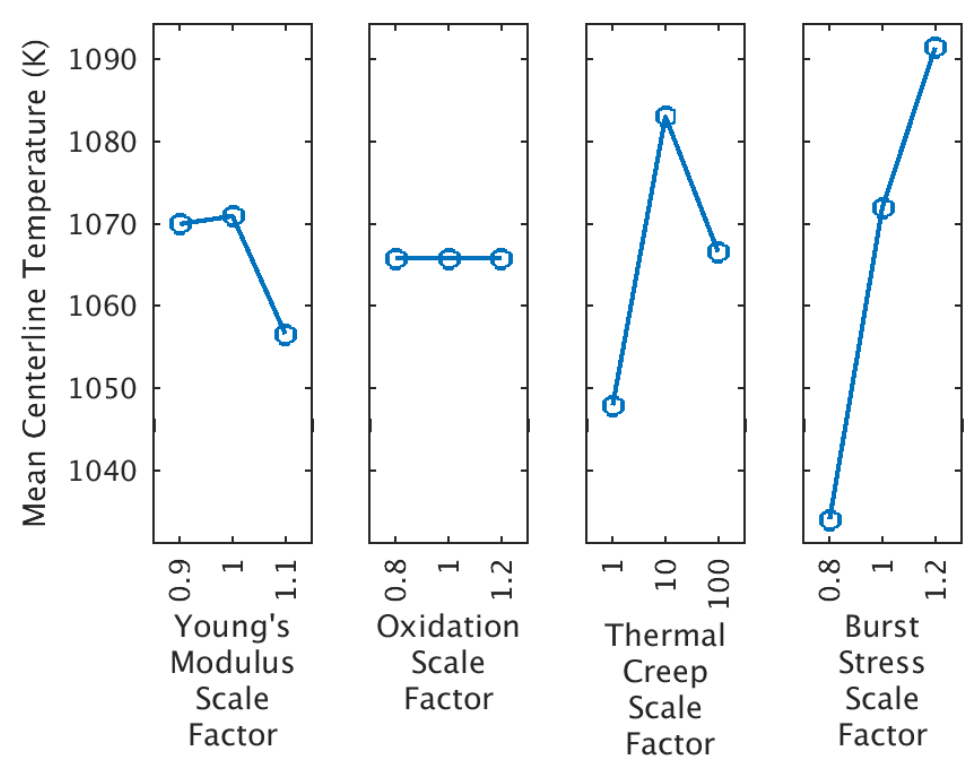

Figure 6.18: Main effects plot for centerline temperature at the end of the LOCA transient for the $\mathrm{UO}_{2} / \mathrm{FeCrAl}$ rodlet.

The hoop strain main effects plots are illustrated in Figure 6.19. The behavior is essentially identical at the inner and outer cladding surfaces. It should be noticed that the strains obtained from the main effects are considerably higher than that obtained in the nominal case shown for the $\mathrm{UO}_{2} / \mathrm{FeCrAl}$ rodlet in Figure 6.16 with the exception of the cases that had a thermal creep 
scaling factor of 1.0. This observation clearly shows the effect of the thermal creep scaling factor on the strain behavior during the high temperature observed in a LOCA. Thus, at high temperature, by scaling the thermal creep scaling factor by two orders of magnitude ballooning behavior similar to nominal Zircaloy-4 can be seen. Therefore, it is imperative to perform additional experiments on $\mathrm{FeCrAl}$ alloys to solidify understanding of the thermal creep behavior. The burst stress scale factor also has a positive correlation with the hoop strain because if the stress resulting in failure is increased the cladding will be able to distend more prior to failing.

The last parameter of interest in terms of LOCA sensitivity analysis is the time to burst (failure) from the onset of the LOCA transient. The results for the $\mathrm{UO}_{2} / \mathrm{FeCrAl}$ rodlet are summarized in Figure 6.20. Increasing the thermal creep scale factor by one order of magnitude increases the time until failure as some cladding distention is permitted and the internal pressure drops reducing the stress exerted on the cladding. However, increasing the factor by another order of magnitude results in significant cladding distention earlier in the transient and the reduced pressure is not enough to offset the high stresses caused by the larger ballooning. As expected, the burst stress is the driving force of the time to burst and has a strong positive correlation with the time to burst. It should be noted that increasing the burst stress in $\mathrm{FeCrAl}$ by $20 \%$ still does not result in the burst time observed for the tradiational $\mathrm{UO}_{2} /$ Zircaloy- 4 rodlet for the nominal LOCA transient described in the previous subsection.

\subsubsection{2 $\mathrm{U}_{3} \mathrm{Si}_{2} /$ Zircaloy-4 Rodlet}

In contrast to the sensitivity analysis completed for the $\mathrm{UO}_{2} / \mathrm{FeCrAl}$ rodlet, the parameters to be varied in the LOCA case for the $\mathrm{U}_{3} \mathrm{Si}_{2} /$ Zircaloy- 4 are not the same as the normal operation study. This is because the fission gas release model for $\mathrm{U}_{3} \mathrm{Si}_{2}$ is not applicable during LOCA transients and the nominal fission gas released is sufficient to study the LOCA effects. The parameters of particular interest in $\mathrm{U}_{3} \mathrm{Si}_{2}$ /Zircaloy-4 analysis are the mechanical and thermal properties of the fuel since it is being treated as elastic. Therefore, the thermal expansion coefficient, Young's modulus and thermal conductivity are varied. Note that the range of thermal conductivity scaling is smaller in this case than for the normal operation case because it is expected to have a strong effect on the results and too large of a variation could lead to convergence problems.

Table 6.5: Parameters varied in the LOCA main effects for the $\mathrm{U}_{3} \mathrm{Si}_{2} / \mathrm{Zircaloy}-4$ rodlet.

\begin{tabular}{lccc}
\hline & Minimum & Intermediate & Maximum \\
\hline Thermal expansion coefficient $\left(\mathrm{K}^{-1}\right)$ & $12 \times 10^{-6}$ & $15 \times 10^{-6}$ & $18 \times 10^{-6}$ \\
Young's modulus (GPa) & 126 & 140 & 154 \\
Thermal conductivity scale factor & 0.95 & 1.0 & 1.05 \\
\hline
\end{tabular}

The sensitivity analysis for the fuel centerline temperature for the $\mathrm{U}_{3} \mathrm{Si}_{2} /$ Zircaloy-4 rodlet is shown in Figure 6.21. It is observed that the variation in thermal expansion coefficient and Young's modulus have very similar trends. Surprisingly, the thermal conductivity has a unique 

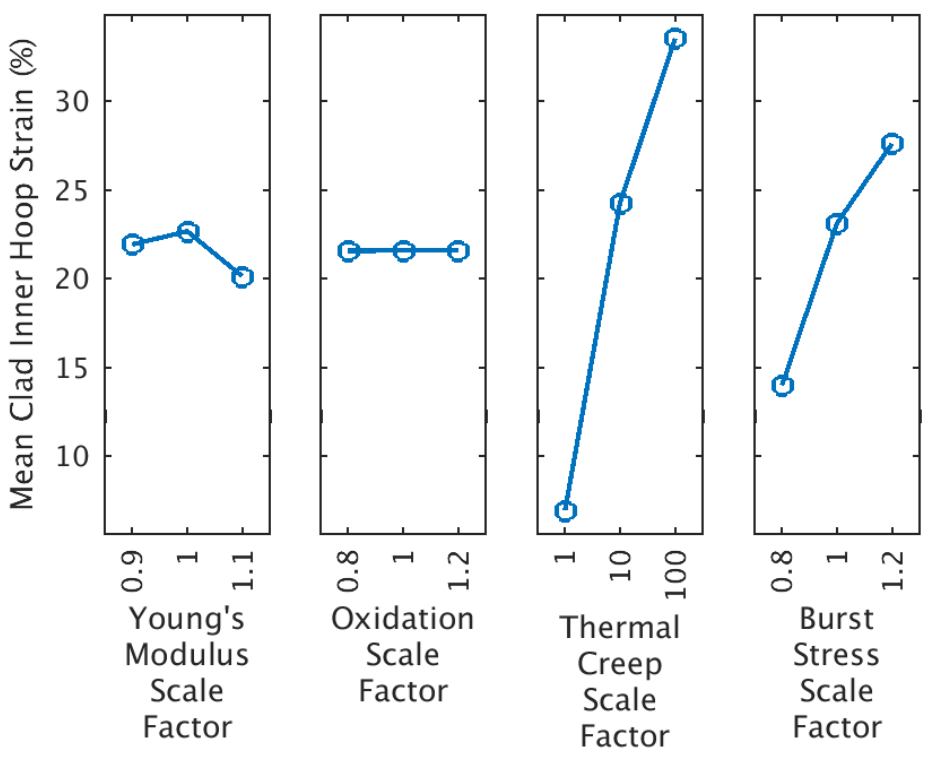

(a)
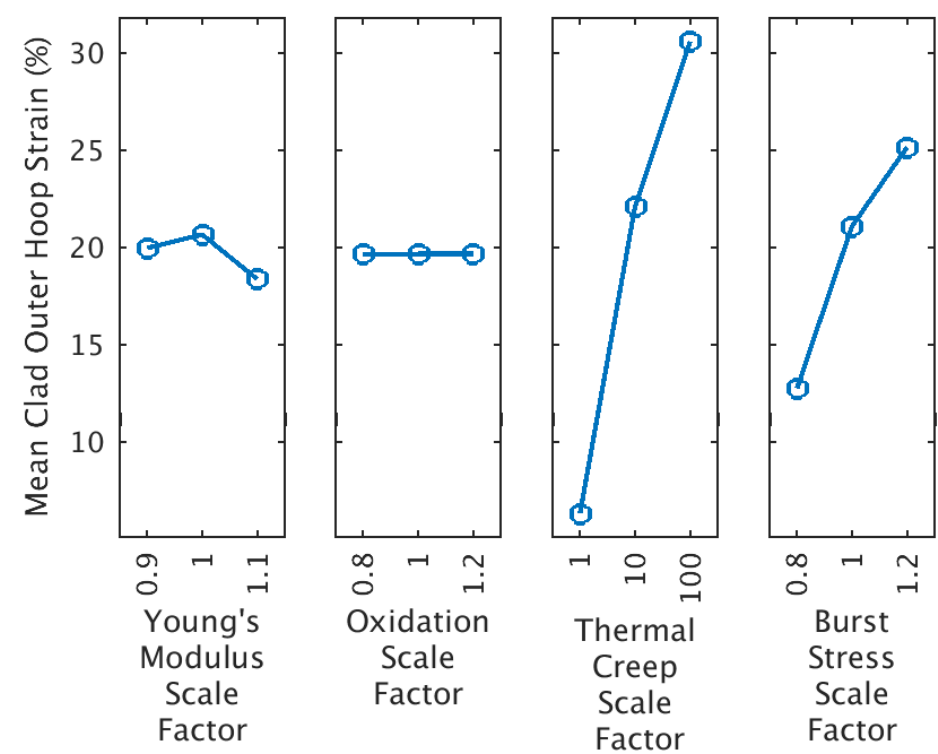

(b)

Figure 6.19: Main effects plots for hoop strain at the (a) inner and (b) outer cladding surface at the end of the LOCA transient for the $\mathrm{UO}_{2} / \mathrm{FeCrAl}$ rodlet.

correlation where the lower and upper bounds have similar temperature where as the unscaled thermal conductivity results in a lower temperature. However, the magnitude difference between 

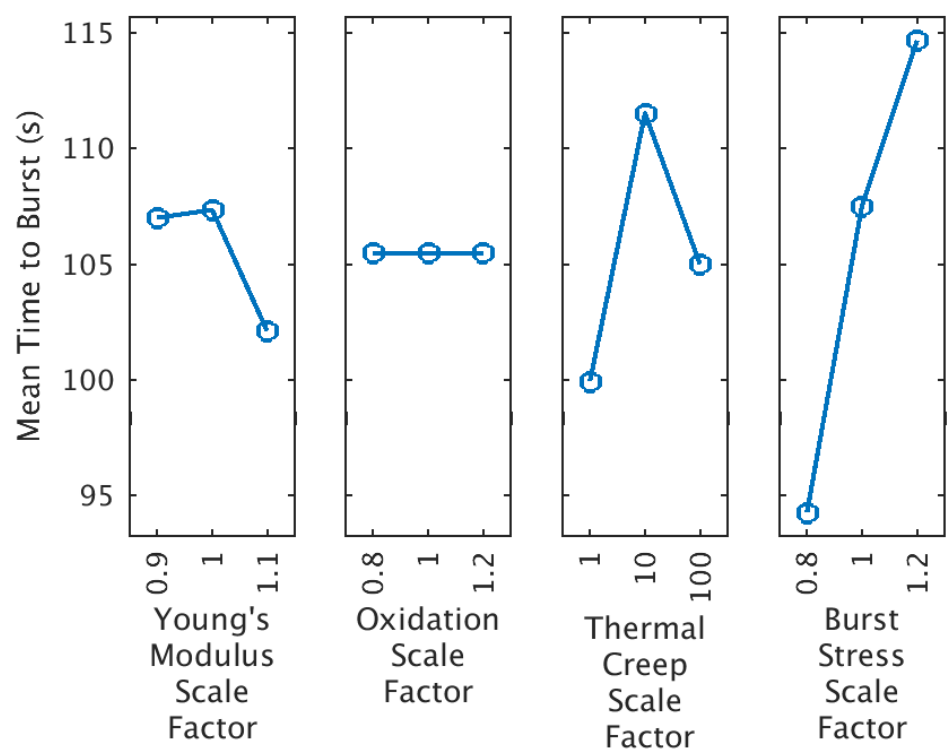

Figure 6.20: Main effects plot for time to burst at the end of the LOCA transient for the $\mathrm{UO}_{2} / \mathrm{FeCrAl}$ rodlet.

the predicted centerline temperature is only approximately $3 \mathrm{~K}$ which indicates that during the postulated LOCA transient here these parameters have very little influence. This is likely attributed to the fact the the power has dropped to the fuel and the increase in fuel temperatures is primarily driven by heat transfer from the outer surface of the cladding, across the gap and into the fuel.

As with the $\mathrm{UO}_{2} / \mathrm{FeCrAl}$ rodlet the trends of the uncertain input parameters on the hoop strain at the inner and outer surface of the cladding are similar with the magnitude of the strains slightly lower at the outer surface as shown in Figure 6.22. As expected the thermal expansion coefficient has a strong positive correlation with the strains as it results in the fuel expanding more and inducing additional strain prior to the LOCA transient. Recall that the variation of the parameters is also applied to the the entire base irradiation prior to the LOCA.

Lastly, the time to burst of the Zircaloy-4 cladding main effects plot for the $\mathrm{U}_{3} \mathrm{Si}_{2} / \mathrm{Zircaloy} 4$ rodlet is illustrated in Figure 6.23. Interestingly the variation in time to burst is only approximately 0.6 seconds. This means that the parameters varied have little effect on the duration of the LOCA transient. This is because during the LOCA transient, the time to burst is primarily driven by the behavior of the cladding as it balloons. 

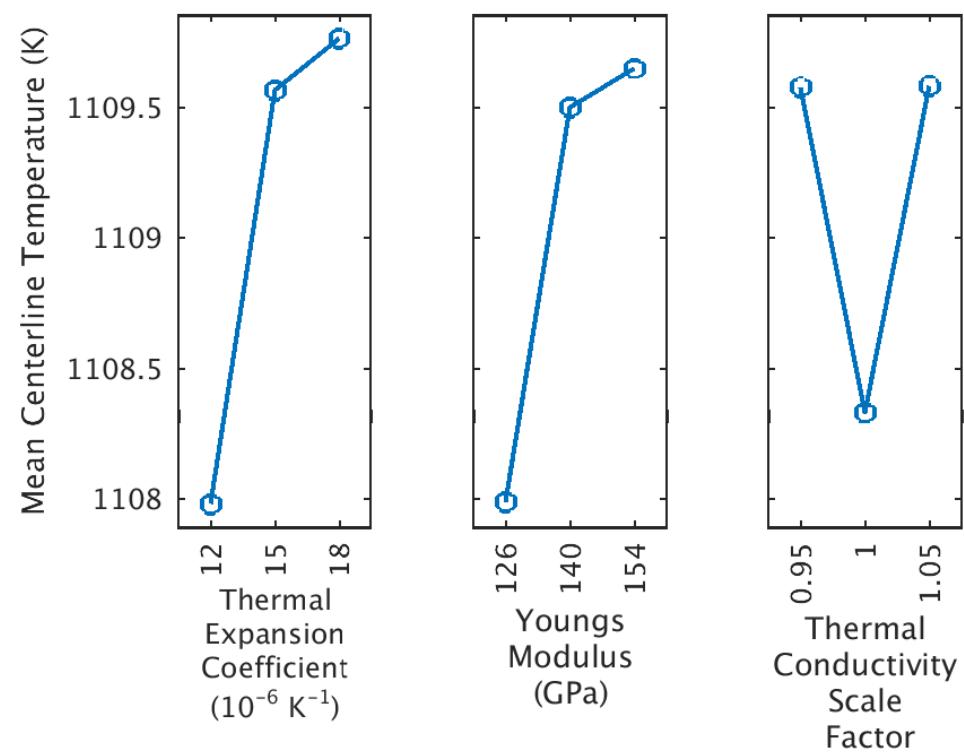

Figure 6.21: Main effects plot for centerline temperature at the end of the LOCA transient for the $\mathrm{U}_{3} \mathrm{Si}_{2} / \mathrm{Zircaloy}-4$ rodlet.

\subsection{Reactivity Insertion Accident}

The last postulated accident to be considered in this work is the Reactivity Insertion Accident (RIA). Early work was completed independently by Pavel Medvedev of the Advanced Fuels Campaign on investigating $\mathrm{U}_{3} \mathrm{Si}_{2}$ behavior during a RIA. This work was confirmed by the first author of this report at a later date. Based upon those results work was completed in collaboration with Brookhaven National Laboratory on using neutronics to inform the RIA pulse in $\mathrm{U}_{3} \mathrm{Si}_{2}$ and assessing the fuel performance behavior. That work was completed for a RIA initiating from hot full power conditions. Given that a RIA initiating from hot zero power is considered the most severe, the initial work by Pavel was revisited using the latest up to date models for the two ATF materials and compared to the traditional $\mathrm{UO}_{2} /$ Zircaloy-4 system.

The RIA scenario used in this study is adopted from an Organisation for Economic Co-operation (OECD) benchmark developed for fuel performance modeling of RIAs in $\mathrm{UO}_{2}$. The cases in the benchmark are relatively simple to allow comparisons between different organizations and their codes. The case chosen from this benchmark for the analysis here was case 3. Figure 6.24 provides a schematic of the problem setup including dimensions and coolant boundary conditions. As before the cladding thickness is adjusted to take account the necessity for thinner cladding in the $\mathrm{FeCrAl}$ case. The total simulation duration is 200 seconds with the RIA pulse beginning at 100 seconds, the maximum $1 \mathrm{MW}$ power to the fuel occuring at 100.03 seconds, and the pulse finishing at 100.06 seconds with a triangular shape. The fuel is assumed to be fresh and contains 

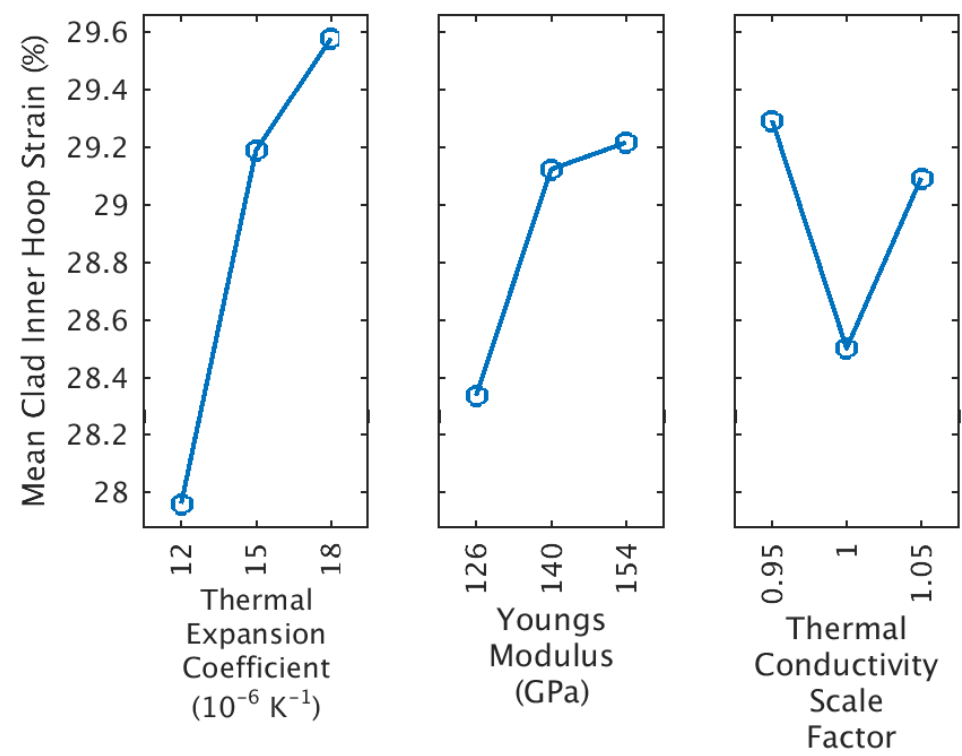

(a)
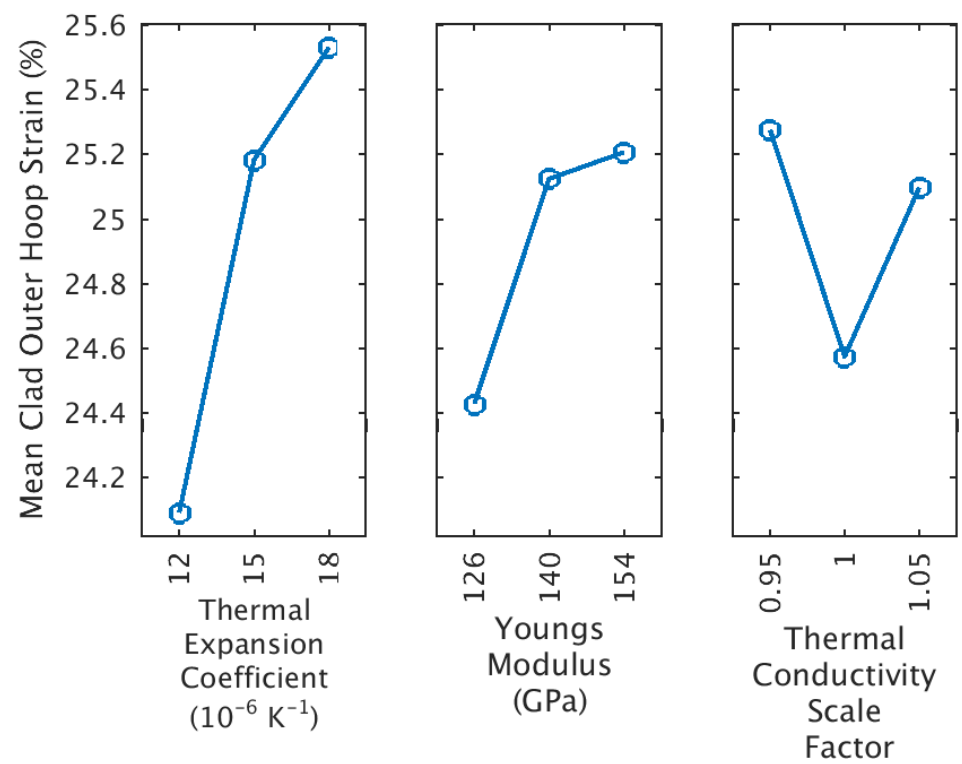

(b)

Figure 6.22: Main effects plots for hoop strain at the (a) inner and (b) outer cladding surface at the end of the LOCA transient for the $\mathrm{U}_{3} \mathrm{Si}_{2} /$ Zircaloy-4 rodlet.

no burnup effects. 

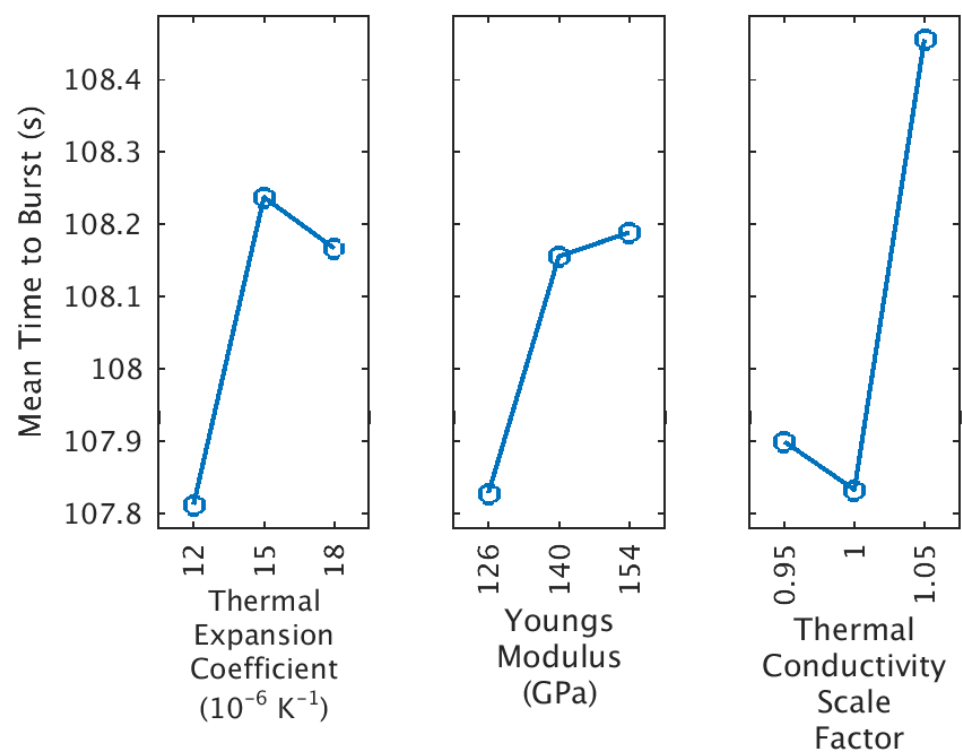

Figure 6.23: Main effects plot for time to burst at the end of the LOCA transient for the $\mathrm{U}_{3} \mathrm{Si}_{2} /$ Zircaloy-4 rodlet.

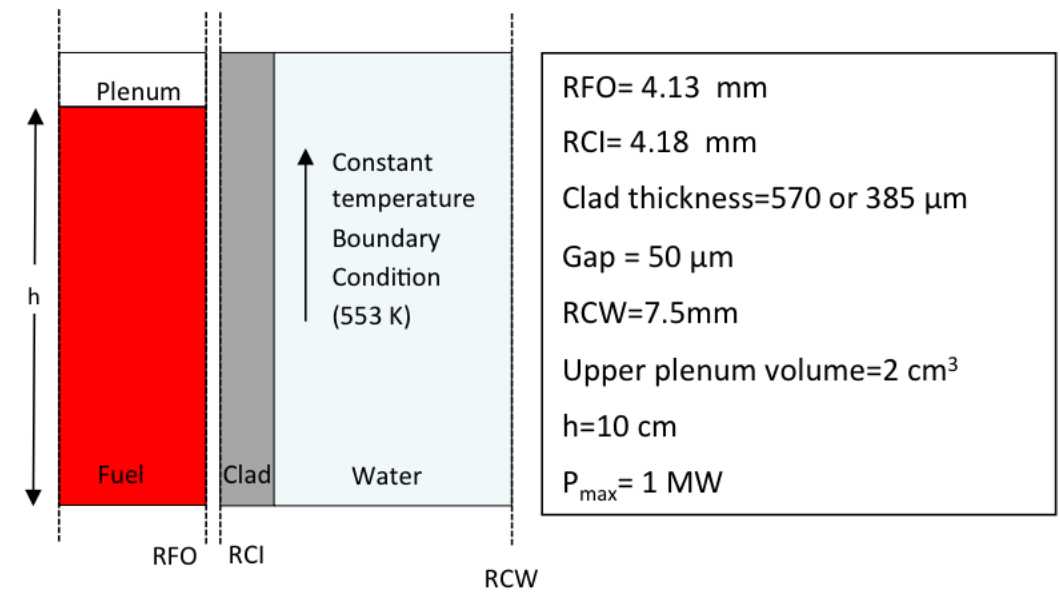

Figure 6.24: Schematic describing the benchmark case used in the RIA analysis.

The centerline temperature, hoop stress and strain at the inner cladding surface, and internal rod pressure are shown in Figures 6.25 through 6.27. Only the mechanical behavior at the inner cladding surface was considered for the RIA transient in contrast to the other analyses in this report because hard contact is expected at the inner surface during the RIA which of interest. The maximum temperature reached by all rodlets during the transient is almost identical (around $2500 \mathrm{~K}$ ) due to the similar specific heat values of the two fuel materials. The temperature in the $\mathrm{U}_{3} \mathrm{Si}_{2} /$ Zircaloy rodlet drops more rapidly after the RIA pulse is finished because the thermal 
conductivity is significantly higher than $\mathrm{UO}_{2}$.

The key observation that should noted is that the $2500 \mathrm{~K}$ centerline temperature attained by $\mathrm{U}_{3} \mathrm{Si}_{2}$ is significantly higher than its melting point of $1938 \mathrm{~K}$. While this is clearly problematic it should be noted that the pulse shape that would occur in $\mathrm{UO}_{2}$ and $\mathrm{U}_{3} \mathrm{Si}_{2}$ are different for the same RIA due to neutronic considerations not taken into account here.

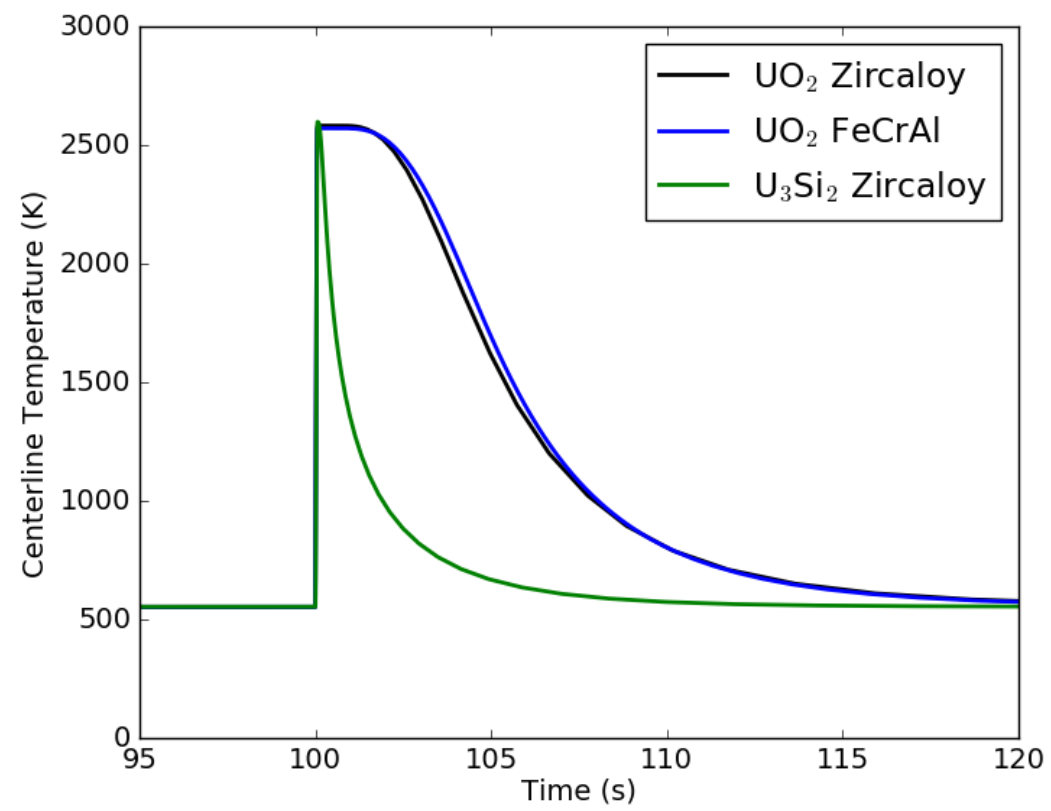

Figure 6.25: Time evolution of the centerline temperature during the RIA transient for all three rodlets.

The hoop strain and stress evolution during the pulse is shown in Figure 6.26. The results show that initially there is some strain in the cladding purely due to thermal expansion. At the initiation of the transient the increased thermal expansion of the fuel leading to solid-to-solid contact causes significant plasticity to occur in the cladding resulting in larger strains. After the transient some of the strain is recovered and stabilizes to a constant value. All rods experience similar stresses. As for the stresses the trends are quite similar among the rodlets except the magnitudes are vastly different. The $\mathrm{UO}_{2} / \mathrm{FeCrAl}$ rod experiences the largest stresses due to the thinner cladding and higher modulus, as expected.

Lastly, the rod internal pressure evolution during the RIA is shown in Figure 6.27. Here, the behavior among the rodlets is quite similar with the maximum pressures reached at the peak of the transient almost being the same. After the transient all rodlets stabilize at a pressure very close to the initial pressure with all converging to a value very close to the initial pressure of the rods prior to the RIA pulse. 


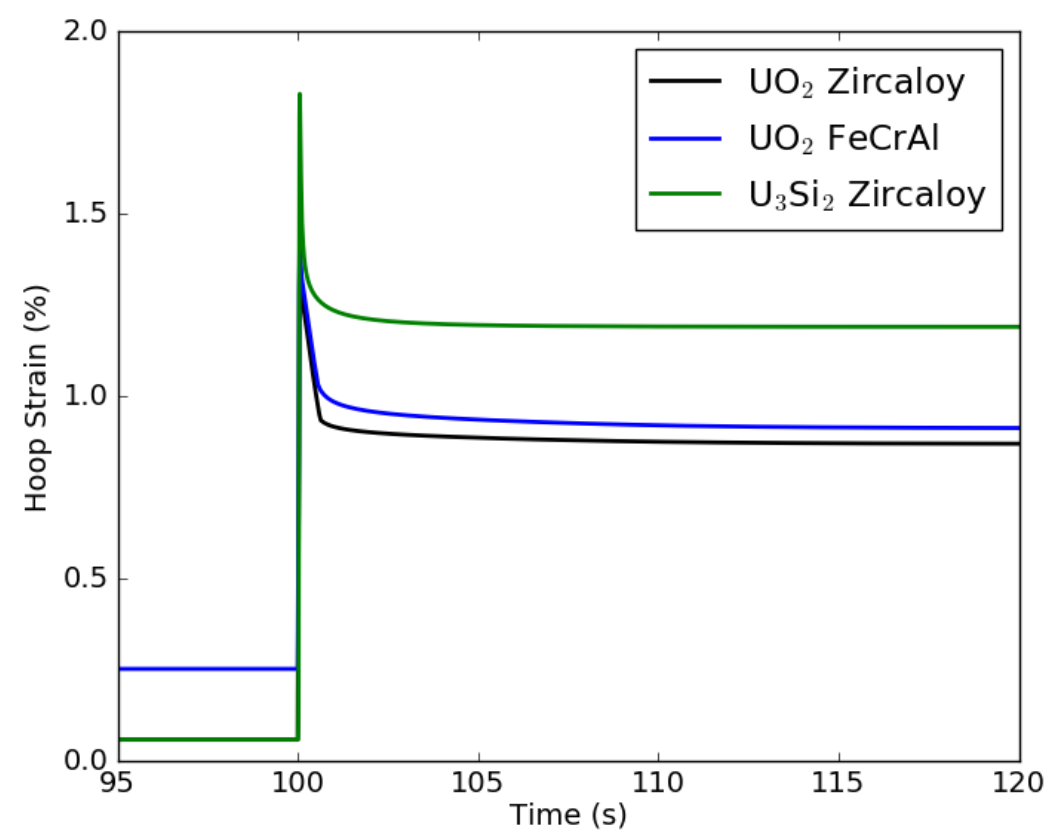

(a)

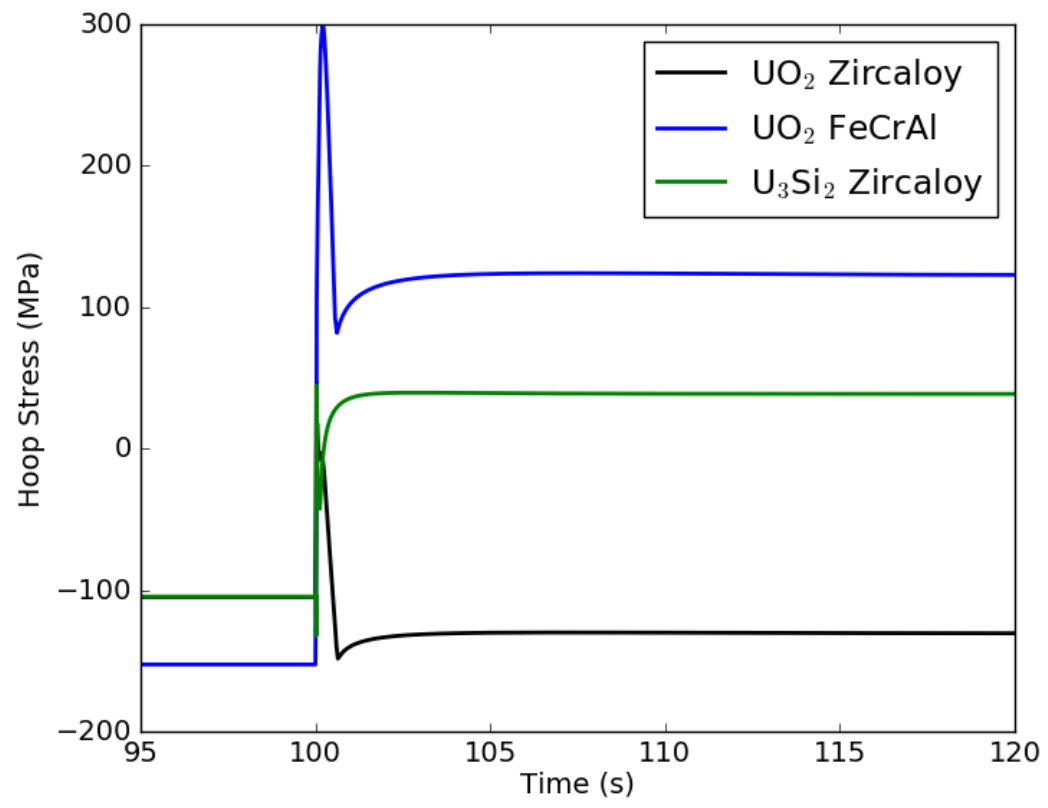

(b)

Figure 6.26: Time evolution of the (a) hoop strain and (b) hoop stress at the inner surface of the cladding during the RIA transient for all three rodlets. 


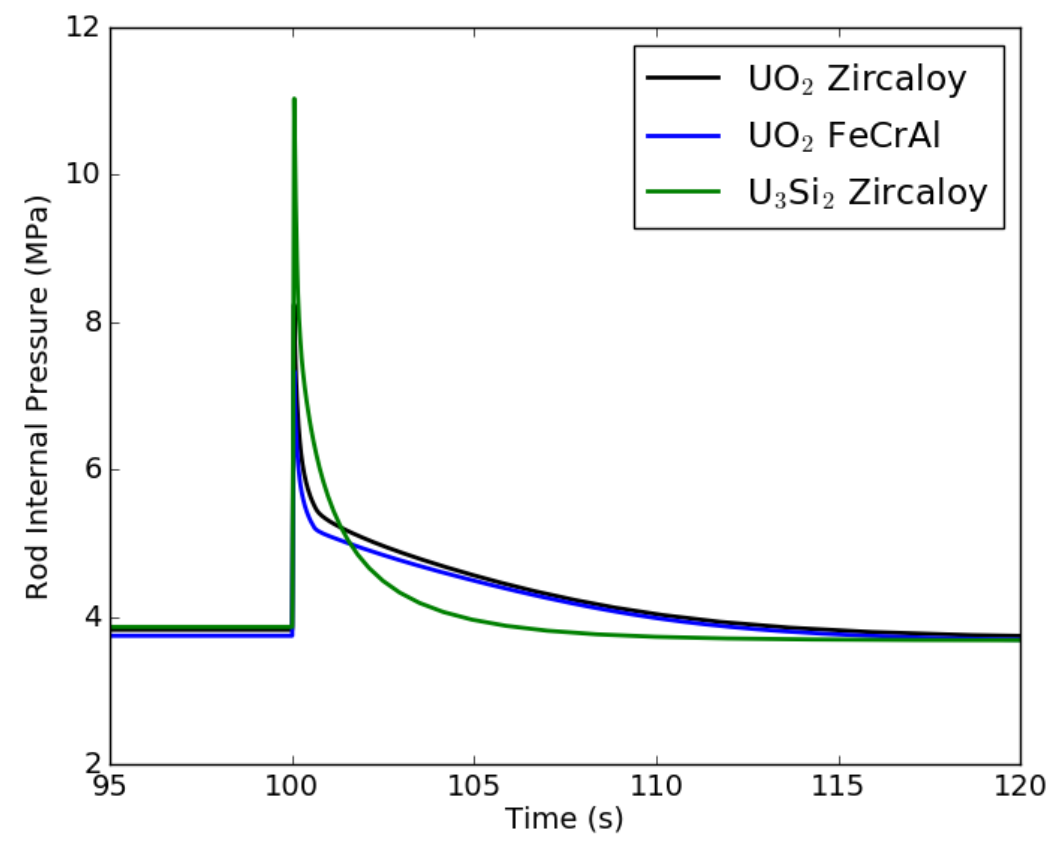

Figure 6.27: Time evolution of the internal rod pressure during the RIA transient for all three rodlets. 


\section{Collaborations}

The success of the ATF HIP has depended on collaboration among researchers at Idaho National Laboratory, Los Alamos National Laboratory, Argonne National Laboratory, and the University of Tennessee-Knoxville. This group met the challenge to develop a modeling capability for ATF with very limited experimental data. The contributions of these individuals is documented elsewhere and will not be repeated here.

\subsection{University of South Carolina}

Much of the early modeling work for $\mathrm{U}_{3} \mathrm{Si}_{2}$ comes from Travis Knight and his team at the University of South Carolina. In particular, Kallie Metzger, who was an intern for a time at INL, performed the first model development and analysis of $\mathrm{U}_{3} \mathrm{Si}_{2}$ in Bison [12, 42]. Her work served as a starting point for further model development and refinement.

\subsection{Massachusetts Institute of Technology}

Massachusetts Institute of Technology (MIT) heads an Integrated Research Project (IRP) on ATF, "Development of Accident Tolerant Fuel Options for Near Term Applications." Other institutions associated with the IRP include the University of Wisconsin, Texas A\&M University, Pennsylvania State University, ANATECH Corp., and AREVA. Researchers in the IRP have exercised Bison capabilities and developed new capabilities also. Most recently, INL hosted an intern from MIT to investigate chromia doped $\mathrm{UO}_{2}$ fission gas release.

\subsection{Texas A\&M University}

Through a contract with Texas A\&M University, a graduate student has spent time at INL investigating coated cladding performance. This work is directed to accident scenarios and especially RIA events. One unique component of this work is the fact that Bison has been coupled to RELAP-7 to determine the heat transfer characteristics and the cladding outer surface. 


\subsection{Westinghouse}

In late September 2016, a meeting was held in Columbia, South Carolina, to formalize a collaboration between Westinghouse and the NEAMS and CASL DOE programs. Two things were accomplished at the meeting: 1) a so-called "Test Stand" was agreed upon and 2) a Bison workshop was held. In both cases, the focus was on accident tolerant fuel.

Since that meeting, INL and Westinghouse staff have continued to communicate regarding the installation and evaluation of Bison. The aim is to run a full suite of ATF analyses $\left(\mathrm{U}_{3} \mathrm{Si}_{2}\right.$ fuel, SiC-SiC cladding, and coated Zirconium based cladding).

The capabilities of Bison will be the focus of a presentation at the Collaboration for Advanced Research on Accident-tolerant Fuel (CARAT) meeting in October 2017.

\subsection{EPRI}

EPRI (Electric Power Research Institute), INL, and DOE have held a series of joint workshops on ATF as part of EPRI's Fuel Reliability Program. Multiple members of the NEAMS Leadership Council have been invited to these meetings and have had the opportunity to present our work. This connection provided us insight into industry's evolving enthusiasm and priorities in ATF.

\subsection{Advanced Fuels Campaign}

As the principal stakeholder in the NEAMS ATF HIP, the Advanced Fuels Campaign has been a critical source of suggestions, guidance, and feedback during the process of developing and exercising the material models discussed in this report.

The team at Oak Ridge National Laboratory, and particularly Kurt Terrani, has been a vital source of information for $\mathrm{FeCrAl}$ models. The creep and oxidation models are built using information directly from this source. Feedback on the kinds of studies that will be valuable and on the relative importance of different performance questions was also very helpful.

Mike Todoso at Brookhaven National laboratory worked with us to understand and model Reactivity Insertion Accidents with ATF materials during hot full power conditions [61].

The opportunity to present the state of the project at Advanced Fuels Campaign meetings, to receive feedback, and to hear about the state of the testing program also was a source of valuable 
information. 


\section{Conclusions}

As the NEAMS ATF HIP draws to a close it was imperative to document the latest state-ofthe-art material models available for the two proposed ATF materials that were the focus of the project, namely, $\mathrm{FeCrAl}$ cladding, and $\mathrm{U}_{3} \mathrm{Si}_{2}$ fuel. The latest version of the models were then used to investigate the relative response between $\mathrm{UO}_{2} / \mathrm{FeCrAl}$ and $\mathrm{U}_{3} \mathrm{Si}_{2} / \mathrm{Zircaloy}-4$ rodlets with a traditional $\mathrm{UO}_{2} /$ Zircaloy-4 rodlet under normal operating, loss of coolant and reactivity insertion conditions. Sensitivity analyses were also completed for the $\mathrm{UO}_{2} / \mathrm{FeCrAl}$ and $\mathrm{U}_{3} \mathrm{Si}_{2} /$ Zircaloy-4 rodlet for the normal operating and loss of coolant cases.

The results indicate that during normal operation that $\mathrm{UO}_{2} / \mathrm{FeCrAl}$ experiences improved oxidation kinetics and the onset of PCMI is delayed. The $\mathrm{U}_{3} \mathrm{Si}_{2} / \mathrm{Zircaloy}-4$ rodlet experiences approximately $600 \mathrm{~K}$ lower fuel centerline temperatures during normal operation. The sensitivity analyses completed on the $\mathrm{UO}_{2} / \mathrm{FeCrAl}$ rodlet indicate that the thermal creep scale factor has the strongest influence on the results during normal operation but the magnitude of variation is small. In the case of the $\mathrm{U}_{3} \mathrm{Si}_{2}$ /Zircaloy-4 rodlet, the scaling of the power and thermal conductivity had an influence in all fuel performance metrics of interest whereas the fission gas behavior parameters only had a strong influence on the amount of fission gas released.

The nominal LOCA simulation appended to the normal operation base irradiation indicated that the $\mathrm{UO}_{2} / \mathrm{FeCrAl}$ rodlet failed first followed by the $\mathrm{U}_{3} \mathrm{Si}_{2} / \mathrm{Zircaloy}-4$ rodlet, and finally the traditional $\mathrm{UO}_{2} /$ Zircaloy- 4 rodlet. Even though the $\mathrm{UO}_{2} / \mathrm{FeCrAl}$ rodlet failed first the strains experienced are about 6 times less than in the two Zircaloy- 4 clad rodlets meaning that the constriction of the subchannels surrounding the rodlet would be less ensuring that coolability is maintained if reflooding were to occur. The sensitivity analyses on the $\mathrm{UO}_{2} / \mathrm{FeCrAl}$ rodlet during the LOCA indicate that the thermal creep and burst stress scale factors had the greatest influence on the results. For the $\mathrm{U}_{3} \mathrm{Si}_{2} /$ Zircaloy- 4 rodlet it is observed that the varied parameters have little impact on the fuel performance metrics at the end of the LOCA transient. This is because the behavior during the postulated LOCA analyzed in this work is primarily driven by the cladding behavior rather than the fuel.

Lastly, an investigation of the fuel performance behavior under RIA conditions was completed using a case from an OECD benchmark on RIA performance in $\mathrm{UO}_{2}$ fuel. The results of this simple analysis indicated that under the pulse used that $\mathrm{U}_{3} \mathrm{Si}_{2}$ would have centerline melting. The $\mathrm{UO}_{2} / \mathrm{FeCrAl}$ rodlet had very similar behavior as the $\mathrm{UO}_{2} /$ Zircaloy- 4 rodlet.

While significant progress has been made throughout the three years of the HIP by using multi- 
scale multiphysics modeling, more work is required. The next chapter highlights some additional modeling activities that will be undertaken. Further experiments are also required to validate the models. 


\section{Future Work}

Much work remains to be done for both $\mathrm{FeCrAl}$ models and $\mathrm{U}_{3} \mathrm{Si}_{2}$ models. More modeling activities are envisioned for other ATF concepts as well.

\subsection{FeCrAl}

While not complete, the state of the models for $\mathrm{FeCrAl}$ is good. With experimental data, models will of course be improved. Creep models in particular will be refined. More work is needed to understand burst. The limited calculations done to date, and reported elsewhere, indicate that $\mathrm{FeCrAl}$ will burst at similar pressures and times as Zircaloy rods. More experiments and calculations need to be done at different conditions to understand that question better. In addition, when $\mathrm{FeCrAl}$ bursts, it may result in a larger opening than when Zircaloy rods burst [16]. This also needs development.

Considerable work remains to utilize the advanced constitutive modeling capabilities developed at Los Alamos National Laboratory in the VPSC code. Initial coupling of VPSC to Bison has been completed, but this must be exercised and evaluated more fully. It may be possible to use a reduced order model, informed by VPSC but more computationally efficient, to improve $\mathrm{FeCrAl}$ modeling capabilities.

\section{$9.2 \mathrm{U}_{3} \mathrm{Si}_{2}$}

A variety of work remains regarding $\mathrm{U}_{3} \mathrm{Si}_{2}$ models. Thermal conductivity degradation seems to have a small but not insignificant effect on fuel centerline temperature. It may be possible to simplify the model developed by Argonne National Laboratory. Similar work at Idaho National Laboratory needs to be evaluated. Experimental data is needed.

For swelling, current models predict significant swelling, considerably more than seen in $\mathrm{UO}_{2}$. Experimental data is needed here also. This data will need to be separated from thermal expansion and densification. A separation of the data between solid and gaseous swelling is also 
needed. In perhaps no other area investigated in this work has a phenomena been so in need of experimental data.

Fission gas is also being modeled in the near absence of data. The current model is fairly expensive and needs efficiency improvements. This model also predicts swelling, and the ideal situation would be to use the fission gas model for swelling also, as is done for $\mathrm{UO}_{2}$.

A journal paper regarding $\mathrm{U}_{3} \mathrm{Si}_{2}$ modeling is envisioned.

\subsection{Silicon Carbide Cladding}

Silicon carbide cladding has a wide range of open modeling issues. However, as a first step, it is necessary to introduce a temperature and fluence dependent swelling model to Bison. Others (University of Tennessee, for example) have developed such a model. If that work is not committed to Bison, a separate model will need to be created.

Issues associated with microcracking, accident behavior, and PCMI are also areas for computational research.

\subsection{Coated Cladding}

Coated Zircaloy cladding is being pursued by several institutions, including AREVA [20]. Early indications are that a thin $(10-20 \mu \mathrm{m})$ chromium coating has a negligible effect on cladding deformation. This is in contrast to preliminary analysis work done. This clearly requires further investigation. A creep model for chromium may be needed.

Given the thin coating layer, it may be helpful to employ mesoscale modeling to understand the character of the Zircaloy/chromium interface.

\subsection{Doped $\mathrm{UO}_{2}$}

Chromium-doped $\mathrm{UO}_{2}$ is being actively developed. It appears that this fuel is more compliant with better fission gas retention than conventional $\mathrm{UO}_{2}$. Fission gas release modeling can be done without much difficulty since the same model can be used with only the diffusion coefficient and grain size changed. Mechanical models are also straightforward given the appropriate mechanical property data. 


\section{Acknowledgements}

Funding for this work is from the DOE Nuclear Energy Advanced Modeling and Simulation program through the Accident Tolerant Fuel High Impact Problem. The submitted manuscript has been authored by a contractor of the U.S. Government under Contract DE-AC07-05ID14517. Accordingly, the U.S. Government retains a non-exclusive, royalty free license to publish or reproduce the published form of this contribution, or allow others to do so, for U.S. Government purposes. 


\section{Bibliography}

[1] S. Bragg-Sitton, B. Merrill, M. Teague, L. Ott, K. Rob, M. Farmer, M. Billone, R. Montgomery, C. Stanek, M. Todosow, and N. Brown. Advanced fuels campaign light water reactor accident tolerant fuel performance metrics. Technical Report INL/EXT-13-29957, Idaho National Laboratory, 2014.

[2] R. L. Williamson, J. D. Hales, S. R. Novascone, M. R. Tonks, D. R. Gaston, C. J. Permann, D. Andrs, and R. C. Martineau. Multidimensional multiphysics simulation of nuclear fuel behavior. Journal of Nuclear Materials, 423:149-163, 2012.

[3] J. D. Hales, R. L. Williamson, S. R. Novascone, D. M. Perez, B. W. Spencer, and G. Pastore. Multidimensional multiphysics simulation of TRISO particle fuel. 443:531-543, 2013.

[4] J. D. Hales, S. R. Novascone, B. W. Spencer, R. L. Williamson, G. Pastore, and D. M. Perez. Verification of the BISON fuel performance code. Annals of Nuclear Energy, 71:81-90, 2014.

[5] M. R. Tonks, D. Gaston, P. C. Millett, D. Andrs, and P. Talbot. An object-oriented finite element framework for multiphysics phase field simulations. Computational Materials Science, 51:20-29, 2012.

[6] J. T. White, A. T. Nelson, J. T. Dunwoody, D. D. Byler, D. J. Safarik, and K. J. McClellan. Thermophysical properties of $\mathrm{U}_{3} \mathrm{Si}_{2}$ to 1773K. Journal of Nuclear Materials, 464:275$280,2015$.

[7] J. M. Harp, L. He, R. E. Hogan, and A. R. Wagner. Corrosion and interdiffusion studies of $\mathrm{U}_{3} \mathrm{Si}_{2}$. In Proceedings of TopFuel, Boise, ID, USA, 2016.

[8] E. S. Wood, J. T. White, D. D. Byler, and A. T. Nelson. The synthesis and air oxidation behavior of U-Si-Al and U-Si-B compositions. In Proceedings of TopFuel, Boise, ID, USA, 2016.

[9] J. T. White, E. S. Wood, J. T. Dunwoody, and A. T. Nelson. State of knowledge and challenges of U-Si compounds for use in light water reactor accident tolerant fuel designs. In Proceedings of TopFuel, Boise, ID, USA, 2016.

[10] D. T. Goddard, D. P. Mathers, D. G. Eaves, P. Xu, E. J. Lahoda, and J. M. Harp. Manufacturability of $\mathrm{U}_{3} \mathrm{Si}_{2}$ and its high temperature oxidation behavior. In Proceedings of TopFuel, Boise, ID, USA, 2016. 
[11] M. R. Finlay, G. L. Hofman, and J. L. Snelgrove. Irradiation behaviour of uranium silicide compounds. Journal of Nuclear Materials, 325:118-128, 2004.

[12] K. E. Metzger, T. W. Knight, and R. L. Williamson. Model of $\mathrm{U}_{3} \mathrm{Si}_{2}$ fuel system using BISON fuel code. In Proceedings of the International Congress on Advances in Nuclear Power Plants - ICAPP 2014, Charlotte, NC, April 6-9 2014.

[13] R. E. Stachowski, R. B. Rebak, W. P. Gassmann, and J. Williams. Progress of GE development of accident tolerant fuel FeCrAl cladding. In Proceedings of TopFuel, Boise, ID, USA, 2016.

[14] K. A. Terrani, S. J. Zinkle, and L. L. Snead. Advanced oxidation-resistant iron-based alloys for LWR fuel cladding. Journal of Nuclear Materials, 448:420-435, 2014.

[15] Xunxiang Hu, Kurt A. Terrani, Brian D. Wirth, and Lance L. Snead. Hydrogen permeation in fecral alloys for LWR cladding application. Journal of Nuclear Materials, 461:282-291, 2015.

[16] Caleb P. Massey, Kurt A. Terrani, Sebastien N. Dryepondt, and Bruce A. Pint. Cladding burst behavior of Fe-based alloys under LOCA. Journal of Nuclear Materials, 470:128138, 2016.

[17] E. Lahoda, S. Ray, F. Boylan, P. Xu, and R. Jacko. SiC cladding corrosion and mitigation. In Proceedings of TopFuel, Boise, ID, USA, 2016.

[18] Weon-Ju Kim, Daejong Kim, and Ji Yeon Park. Fabrication and material issues for the application of SiC composites to LWR fuel cladding. Nuclear Engineering and Technology, 45(4):565-572, 2013.

[19] Yutai Katoh, Lance L. Snead, Ting Cheng, Chunghao Shih, W. Daniel Lewis, Takaaki Koyanagi, Tatsuya Hinoki, Charles H. Henager Jr., and Monica Ferraris. Radiation-tolerant joining technologies for silicon carbide ceramics and composites. Journal of Nuclear Materials, 448(13):497-511, 2014.

[20] J. Bischoff, C. Vauglin, C. Delafoy, P. Barberis, D. Perche, B. Guerin, J.-P. Vassault, and J.-C. Brachet. Development of Cr-coated zirconium alloy cladding for enhanced accident tolerance. In Proceedings of TopFuel, Boise, ID, USA, 2016.

[21] K. A. Terrani, L. L. Snead, and J. C. Gehin. Microencapsulated fuel technology for commercial light water and advanced reactor application. 427(13):209 - 224, 2012.

[22] M. R. Tonks, P. C. Millett, P. Nerikar, S. Du, D. Andersson, C. R. Stanek, D. Gaston, D. Andrs, and R. Williamson. Multiscale development of a fission gas thermal conductivity model: Coupling atomic, meso and continuum level simulations. 440:193-200, 2013.

[23] M. R. Tonks, X. Y. Liu, D. Andersson, D. Perez, A. Chernatynskiy, G. Pastore, C. R. Stanek, and R. L. Williamson. Development of a multiscale thermal conductivity model for fission gas in $\mathrm{UO}_{2}$. 469:89-98, 2016. 
[24] X. M. Bai, M. R. Tonks, Y. Zhang, and J. D. Hales. Multiscale modeling of thermal conductivity of high burnup structures in $\mathrm{UO}_{2}$ fuels. 470:208-215, 2016.

[25] Yinbin Miao, Kyle A. Gamble, David Andersson, Bei Ye, Zhi-Gang Mei, Gerard Hofman, and Abdellatif M. Yacout. Gaseous swelling of u3si2 during steady-state lwr operation: A rate theory investigation. Nuclear Engineering and Design, 322:336 - 344, 2017.

[26] C. N. Tomé and R. A. Lebesohn. Manual for code Visco-Plastic Self-Consistent (VPSC). Technical Report LA-CC-99-72, Los Alamos National Laboratory, 2009.

[27] J. T. White, A. T. Nelson, J. T. Dunwoody, D. J. Safarik, and K. J. McClellan. Corrigendum to Thermophysical Properties of $\mathrm{U}_{3} \mathrm{Si}_{2}$ to 1777 K. Journal of Nuclear Materials, 484:386387, 2017.

[28] Y. Miao, K. A. Gamble, D. Andersson, B. Ye, Z. Mei, G. Hofman, and A. M. Yacout. Gaseous swelling of $\mathrm{U}_{3} \mathrm{Si}_{2}$ during steady-state LWR operation: A rate theory investigation. Nuclear Engineering and Design, 322:336-344, 2017.

[29] Y. Rashid, R. Dunham, and R. Montgomery. Fuel analysis and licensing code: FALCON MOD01. Technical Report EPRI 1011308, Electric Power Research Institute, December 2004.

[30] J.L Snelgrove M. R. Finlay, G. L. Hofman. Irradiation behaviour of uranium silicide compounds. Journal of Nuclear Materials, 325:118-128, 2004.

[31] G. L. Hofman and W. S. Ryu. Detailed Analysis of Uranium Silicide Dispersion Fuel Swelling. Technical Report CONF-8909141-10, Argonne National Laboratory, 1989.

[32] H. Shimizu. The properties and irradiation behavior of $\mathrm{U}_{3} \mathrm{Si}_{2}$. Technical Report NAA-SR10621, Atomics International, 1965.

[33] D. R. Olander and D. Wongsawaeng. Re-solution of fission gas - A review: Part I. Intragranular bubbles. Journal of Nuclear Materials, 354:94-109, 2006.

[34] C. Matthews, D.A. Andersson, and C. Unal. Radiation re-solution calculation in uraniumsilicide fuels. Technical report, Los Alamos National Laboratory, 2016.

[35] M. S. Veshchunov. On the theory of fission gas bubble evolution in irradiated $\mathrm{UO}_{2}$ fuel. Journal of Nuclear Materials, 277:67-81, 2000.

[36] D. Pizzocri, C. Rabiti, L. Luzzi, T. Barani, P. Van Uffelen, and G. Pastore. PolyPole-1: An accurate numeical algorithm for intra-granular fission gas release. Journal of Nuclear Materials, 478:333-342, 2016.

[37] M.V. Speight and W. Beere. Vacancy potential and void growth on grain boundaries. Metal Science, 9:190-191, 1975.

[38] D. Pizzocri, F. Cappia, V. V. Rondinella, and P. Van Uffelen. Preliminary model for the pore growth in the HBS. Technical report, JRC103064, European Commission, Directorate for Nuclear Safety and Security, JRC-Karlsruhe, 2016. 
[39] D.A. Andersson. Density functional theory calculations of the defect and fission gas properties in U-Si fuels. Technical report, Los Alamos National Laboratory, 2017.

[40] G. Pastore, L. Luzzi, V. Di Marcello, and P. Van Uffelen. Physics-based modelling of fission gas swelling and release in $\mathrm{UO}_{2}$ applied to integral fuel rod analysis. Nuclear Engineering and Design, 256:75-86, 2013.

[41] R.J. White. The development of grain-face porosity in irradiated oxide fuel. Journal of Nuclear Materials, 325:61-77, 2004.

[42] K. E. Metzger. Analysis of Pellet Cladding Interaction and Creep of $U_{3} \mathrm{Si}_{2}$ Fuel for Use in Light Water Reactors. PhD thesis, University of South Carolina, 2016. Retrieved from http://scholarcommons.sc.edu/etd/3811.

[43] Y. Yamamoto, Y. Yang, K.G. Field, K. Terrani, B.A. Pint, and L.L. Snead. Letter report documenting progress of second generation ATF FeCrAl alloy fabrication. Technical Report ORNL/LTR-2014/219,2014, Oak Ridge National Laboratory, 2014.

[44] Y. Yamamoto, B.A. Pint, K.A. Terrani, K.G. Field, Y. Yang, and L.L. Snead. Development and property evaluation of nuclear grade wrought $\mathrm{FeCrAl}$ fuel cladding for light water reactors. Journal of Nuclear Materials, 467:703-716, 2015.

[45] Z. T. Thompson, K. A. Terrani, and Y. Yamamoto. Elastic Modulus Measurement of ORNL ATF FeCrAl Alloys. Technical Report ORNL/TM-2015/632, Oak Ridge National Laboratory, October 2015.

[46] Y. Yano, T. Tanno, Y. Sekio, H. Oka, S. Ohtsuka, T. Uwaba, and T. Kaito. Tensile properties and hardness of two types of $11 \mathrm{cr}$-ferritic/martensitic steel after aging up to $45,000 \mathrm{~h}$. Nuclear Materials and Energy, 000:1-7, 2016.

[47] Kanthal APMT (Tube) Datasheet. http://kanthal.com/en/products/materialdatasheets/tube/kanthal-apmt/, 2012.

[48] M. Niffenegger and K. Reichlin. The proper use of thermal expansion coefficients in finite element calculations. Nuclear Engineering and Design, 243:356-359, 2012.

[49] K. A. Terrani, T. M. Karlsen, and Y. Yamamoto. Input correlations for irradiation creep of FeCrAl and $\mathrm{SiC}$ based on in-pile Halden test results. Technical Report ORNL/TM2016/191, ORNL, May 2016.

[50] S. R. J. Saunders, H. E. Evans, M. Li, D. D. Gohil, and S. Osgerby. Oxidation growth stresses in an alumina-forming ferritic steel measured by creep deflection. Oxidation of Metals, 48:189-200, 1997.

[51] K. G. Field, X. Hu, K. C. Littrell, Y. Yamamoto, and L. L. Snead. Radiation tolerance of neutron-irradiated model fe-cr-al alloys. Journal of Nuclear Materials, 465:746-755, 2015. 
[52] K.A. Terrani, B.A. Pint, K.A. Unocic, Y. Yang, C.M. Silva, H.M. Meyer III, and R.B. Rebak. Uniform corrosion of FeCrAl alloys in LWR coolant environments. Journal of Nuclear Materials, 479:36-47, 2016.

[53] K. A. Gamble, T. Barani, D. Pizzocri, J. D. Hales, K. A. Terrani, and G. Pastore. An investigation of $\mathrm{FeCrAl}$ cladding behavior under normal operating and loss of coolant conditions. Journal of Nuclear Materials, 491:55-66, 2017.

[54] K. A. Gamble and J. D. Hales. Nuclear Energy Advanced Modeling and Simulation (NEAMS) high impact problem: FeCrAl modeling capabilities. Technical Report INL/EXT-17-43118, 2017.

[55] K. A. Gamble and J. D. Hales. Nuclear Energy Advanced Modeling and Simulation (NEAMS) high impact problem: U3Si2 modeling capabilities. Technical Report INL/EXT17-43117, 2017.

[56] Kyle A. Gamble and Jason D. Hales. Preliminary evaluation of FeCrAl cladding and U-Si fuel for accident tolerant fuel concepts. In TopFuel 2015 Conference Proceedings, Zurich, Switzerland, 13-17 September 2015.

[57] Kyle A. Gamble and Jason D. Hales. Preliminary modeling of accident tolerant fuel concepts under accident conditions. In TopFuel 2016 Conference Proceedings, Boise, ID, 11-16 September 2016.

[58] Jason D. Hales and Kyle A. Gamble. Multiscale modeling of accident tolerant fuels under the NEAMS ATF program. In 2016 ANS Annual Meeting, New Orleans, LA, 12-16 June 2016.

[59] J. D. Hales and K. A. Gamble. Modeling accident tolerant fuel concepts. In Enlarged Halden Programme Group Meeting, Oslo/Fornebu, Norway, 8-13 May 2016.

[60] B. M. Adams, K. R. Dalbey, M. S. Eldred, L. P. Swiler, W. J. Bohnhoff, J. P. Eddy, D. M. Vigil, P. D. Hough, and S. Lefantzi. DAKOTA: A multilevel parallel object-oriented framework for design optimization, parameter estimation, uncertainty quantification, and sensitivity analysis. Version 5.2 user's manual. Technical Report SAND2010-2183, Sandia National Laboratories, 2011.

[61] L. Y. Cheng, A. Cuadra, M. Todosow, K. A. Gamble, and P. G. Medvedev. Performance of $\mathrm{U}_{3} \mathrm{Si}_{2}$ fuel in a reactivity insertion accident. In TopFuel 2016 Conference Proceedings, Boise, ID, 11-16 September 2016. 